University of Louisville

ThinkIR: The University of Louisville's Institutional Repository

Electronic Theses and Dissertations

$5-2013$

\title{
Influential variables on the timing of help-seeking behavior in lung cancer patients.
}

Lisa Carter-Harris

University of Louisville

Follow this and additional works at: https://ir.library.louisville.edu/etd

Part of the Nursing Commons

\section{Recommended Citation}

Carter-Harris, Lisa, "Influential variables on the timing of help-seeking behavior in lung cancer patients." (2013). Electronic Theses and Dissertations. Paper 219.

https://doi.org/10.18297/etd/219

This Doctoral Dissertation is brought to you for free and open access by ThinkIR: The University of Louisville's Institutional Repository. It has been accepted for inclusion in Electronic Theses and Dissertations by an authorized administrator of ThinkIR: The University of Louisville's Institutional Repository. This title appears here courtesy of the author, who has retained all other copyrights. For more information, please contact thinkir@louisville.edu. 
INFLUENTIAL VARIABLES ON THE TIMING OF HELP-SEEKING BEHAVIOR IN LUNG CANCER PATIENTS

\author{
By \\ Lisa Carter-Harris \\ BSN, Spalding University, 1999 \\ MSN, University of Louisville, 2004
}

\begin{abstract}
A Dissertation
Submitted to the Faculty of the

School of Nursing of the University of Louisville

in Partial Fulfillment of the Requirements

for the Degree of
\end{abstract}

Doctor of Philosophy

School of Nursing

University of Louisville

Louisville, Kentucky

May 2013 
(C) Copyright 2013 by Lisa Carter-Harris

All rights reserved 

INFLUENTIAL VARIABLES ON THE TIMING OF HELP-SEEKING BEHAVIOR IN LUNG CANCER PATIENTS

\author{
By \\ Lisa Carter-Harris \\ BSN, Spalding University, 1999 \\ MSN, University of Louisville, 2004
}

A Dissertation Approved on

May 28, 2013

by the following Dissertation Committee:

\begin{tabular}{c}
\hline $\begin{array}{c}\text { Carla P. Hermann, PhD, RN } \\
\text { Dissertation Chair }\end{array}$ \\
\hline Judy Schreiber, PhD, RN \\
\hline M. Celeste Shawler, PhD, RN
\end{tabular}

M. Celeste Shawler, PhD, RN

Jenna Hatcher-Keller, PhD, RN, MPH 


\section{DEDICATION}

This dissertation is dedicated to my mother, Louise Brinson Carter, who believed in me every step of the way even when I did not see the finish line. She gave me the gift of herself as a listening ear, a quiet cheerleader, and an unconditional

presence of love and support. She taught me to never give up on my dreams and that my best would always suffice as long as it was my best. To my father, John Heyward Carter, Sr., who lost his six-year battle with Alzheimer's dementia earlier this year. You taught me that life begins at the end of your comfort zone and ignited a passion for education that has traversed the decades of my life. You are truly missed, but your spirit lives forever within me and all whom you touched. To my husband, Kevin Ned Harris, who has unconditionally supported me through the many hours, days, and years of this process. To my children, Zachary Keaton Harris and Gabriella "Elli" Harris, for always cheering me on this long journey and allowing me the privilege of the best job in the world...Mommy! 


\section{ACKNOWLEDGMENTS}

The following dissertation is the culmination of a long journey of many footsteps and a result of the guidance, support, and friendship of a number of individuals. I would first like to thank my dissertation chair, Dr. Carla Hermann, for her guidance and patience throughout my journey. Dr. Hermann has been a steadfast, guiding force throughout from the very first course to the very last edit. She has graciously provided me with something more valuable than anything tangible...the opportunity to grow and the vision to see new heights that I never imagined. I am forever touched by her compassion, grateful for her patience, and most importantly, thankful for her friendship. Next, I would like to thank my committee members, Dr. Jenna Hatcher-Keller, Dr. Judy Schreiber, and Dr. Celeste Shawler, Dr. Hatcher-Keller replied to a random email from a doctoral student with open arms and has graciously provided experience, knowledge, and direction to this work. I am also immensely grateful for the many hours of time, advice, and supportive words of direction offered by Dr. Schreiber. Finally, from my first semester in the doctoral program to this moment, Dr. Shawler has been a constant presence providing thoughtful feedback, experience, new perspectives, and has helped me to always see the individual in the work and appreciate the lives we touch. I would also like to acknowledge and thank Dr. Lynne Hall for her generosity of time, expert advice, and her 'green pen'. Although not a formal member of my dissertation committee, Dr. Hall graciously 
provided hours of time and wonderful direction with this work. Finally, the faculty and staff at the School of Nursing have been exceptional in their support and instrumental in many ways throughout this dissertation work. I am privileged to have had the opportunity to learn and grow at the University of Louisville.

I would also like to express my deepest gratitude to the individuals living with lung cancer that allowed me into their lives and shared their wonderful stories that helped me to understand my work more completely. You are truly the reason for this work, and I was honored and humbled to work with such wonderful individuals.

I would like to thank all of my friends and family, and I would like to extend a special appreciation to my 'sisters': Glenda Adams, Heather Hardin, Lisa Long, Sheila Steinbrenner, and Xiaorong Wang. We began this journey together as colleagues sitting in a classroom every Monday for hours on end and developed bonds that have resulted in a family. Thank you for your endless encouragement, empowering me through my tears and frustration, providing infinite laughter and smiles, and sharing this journey with me.

Last, but certainly not least, a very special recognition to my husband, Kevin, for his understanding and patience during those times when there was no light at the end of anything. He encouraged me, accepted me, and ran a fourperson household many times solo without complaint. I love you so very much! 


\title{
ABSTRACT \\ INFLUENTIAL VARIABLES ON THE TIMING OF HELP-SEEKING BEHAVIOR IN LUNG CANCER PATIENTS
}

\author{
Lisa Carter-Harris
}

May 28, 2013

Lung cancer kills more people than any other cancer worldwide primarily because it is often diagnosed at an advanced stage. One factor that can influence advanced stage lung cancer diagnosis is delayed help-seeking behavior in individuals with symptoms suggestive of lung cancer. Delayed helpseeking behavior has been investigated in acute cardiovascular events and breast cancer, but there is little evidence on specific factors that influence the timing of help-seeking behavior in lung cancer patients. The purpose of this study was to explore the influence of healthcare system distrust, lung cancer stigma, and smoking status on the timing of help-seeking behavior in individuals with symptoms suggestive of lung cancer. This study employed a descriptive, crosssectional design with 93 participants using the Revised Healthcare System Distrust Scale, Cataldo Lung Cancer Stigma Scale, and investigator-developed Timing of Help-Seeking Behavior and Demographic Questionnaire to assess the variables of interest. 
Hierarchical linear regression was used to assess the ability of healthcare system distrust, lung cancer stigma, and smoking status to predict greater time to seek help in lung cancer patients, after controlling for annual income, perceived financial status, ethnicity, and social desirability. In Step 1, ethnicity and perceived financial status explained $10 \%$ of the variance in time to seek help. After entry of healthcare system distrust, lung cancer stigma, and smoking status at Step 2, the total variance explained by the model as a whole was $23 \%$ of the variance in the time to seek help in individuals with symptoms suggestive of lung cancer. Significant findings from this study include the effect of healthcare system distrust, lung cancer stigma, and ethnicity on help-seeking behavior in individuals with lung cancer symptoms. The findings indicate a critical need for public health awareness that targets increasing awareness of lung cancer symptoms, decreasing lung cancer stigma and healthcare system distrust, recognizing the significant proportion of lung cancer patients whom have never smoked, and decreasing delays in help-seeking behaviors in individuals with symptoms suggestive of lung cancer. 


\section{TABLE OF CONTENTS}

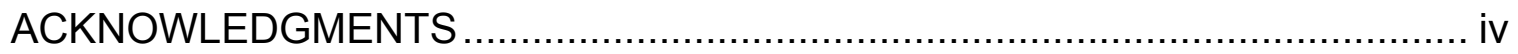

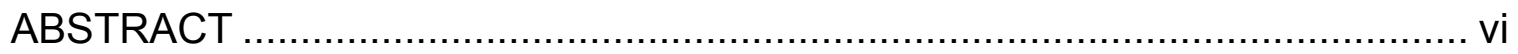

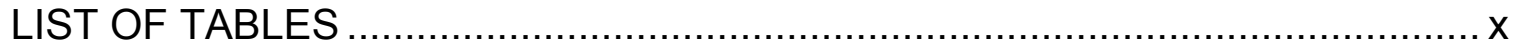

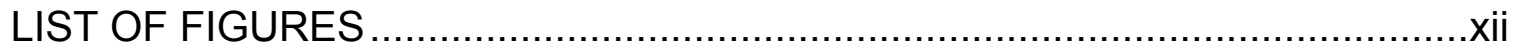

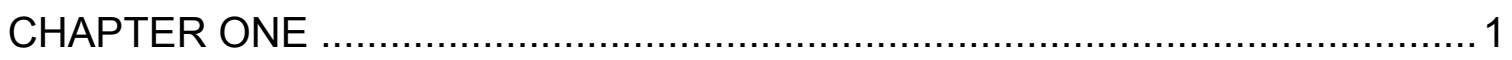

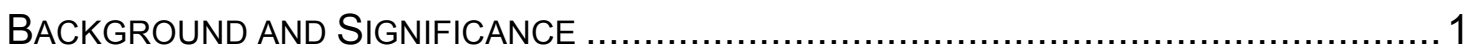

STATEMENT OF THE PROBLEM \& SIGNIFICANCE FOR NURSING ............................. 3

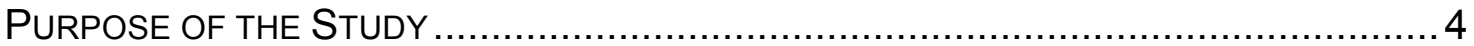

SPECIFIC AIMS AND STUDY HYPOTHESES ................................................... 5

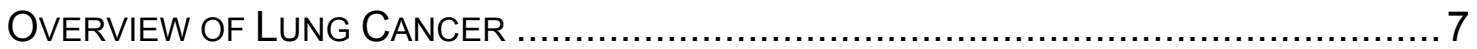

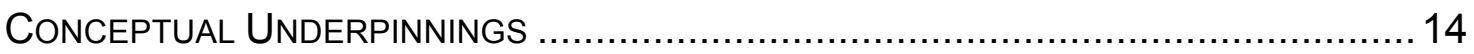

Conceptual Model for Help-Seeking Behavior in Lung Cancer .................... 20

Conceptual Model for Help-Seeking Behavior in Lung Cancer and its Use

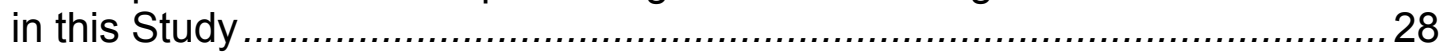

CONCEPTUAL AND OPERATIONAL DEFINITIONS .............................................. 32

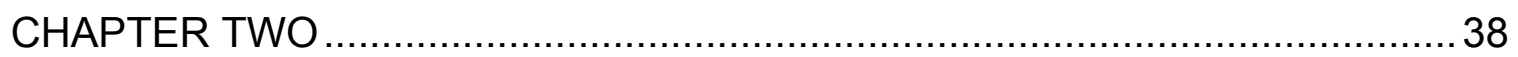

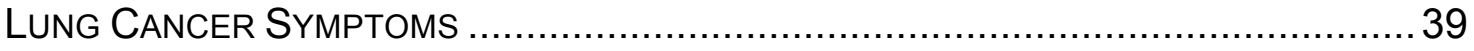

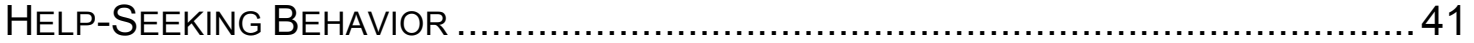

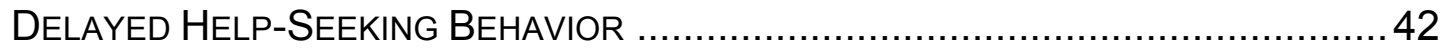

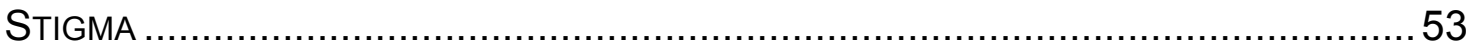

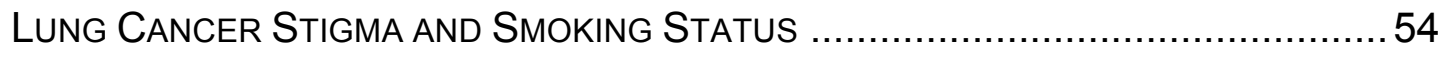

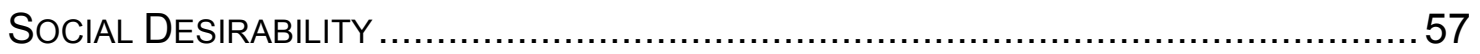

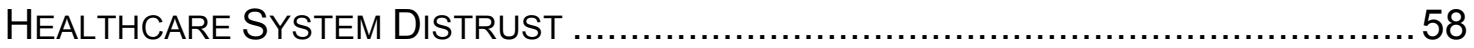

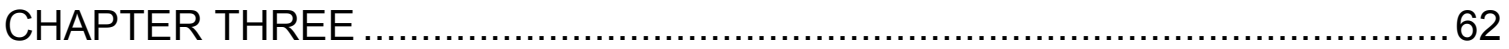

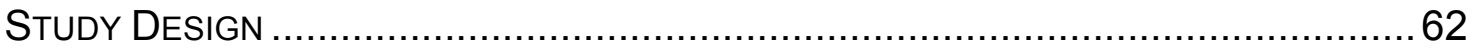

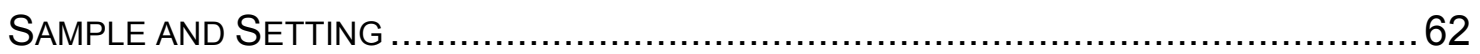

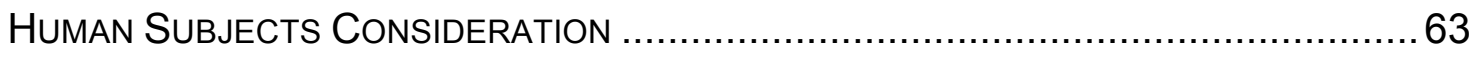

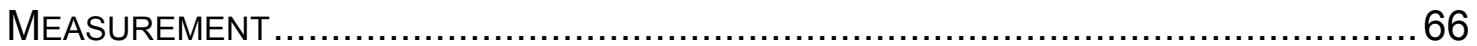

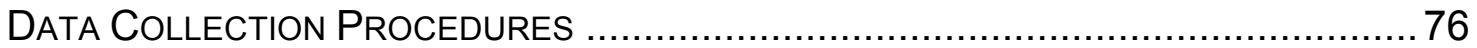

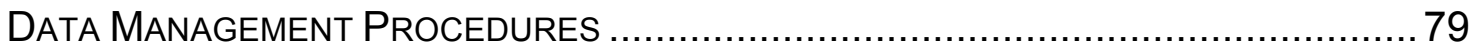

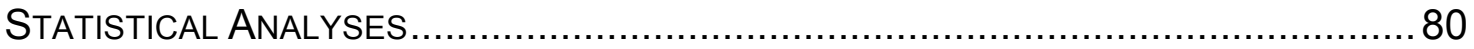

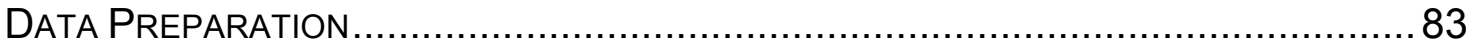

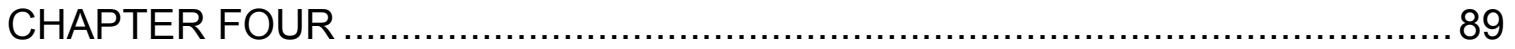

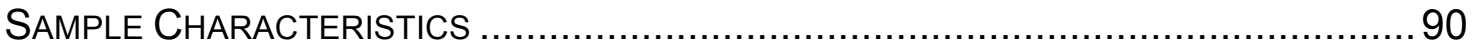




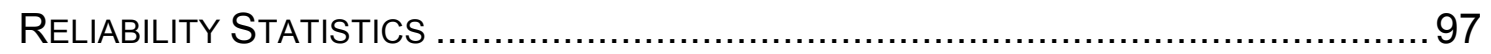

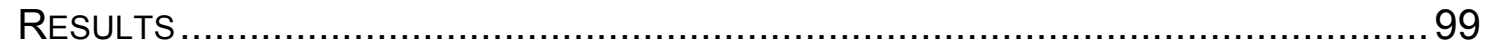

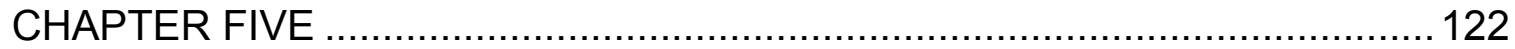

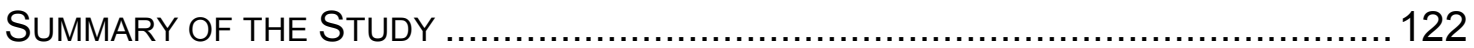

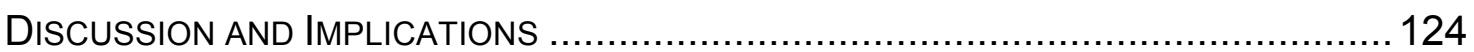

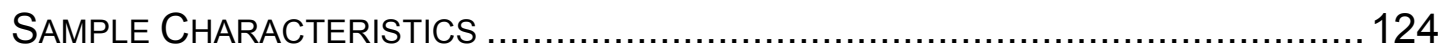

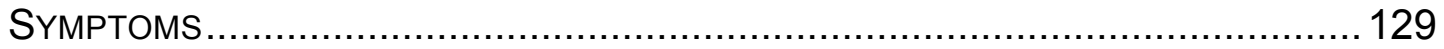

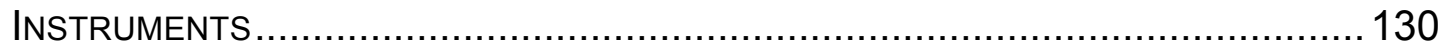

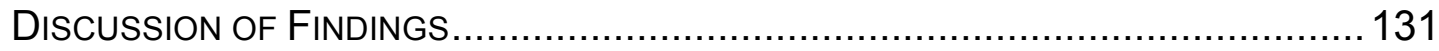

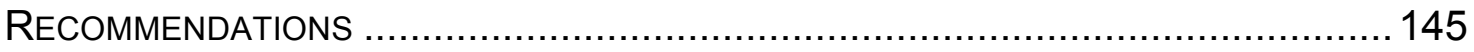

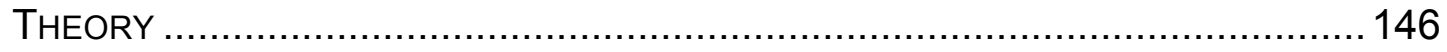

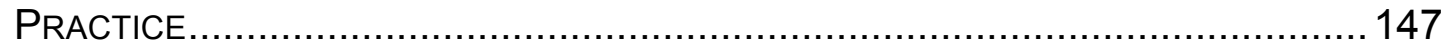

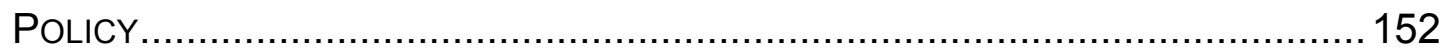

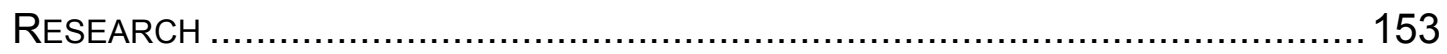

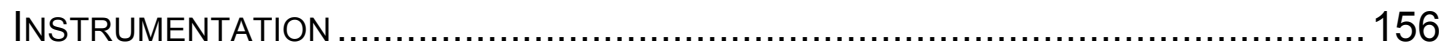

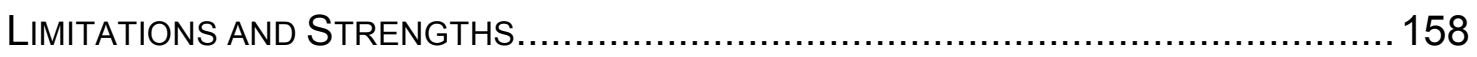

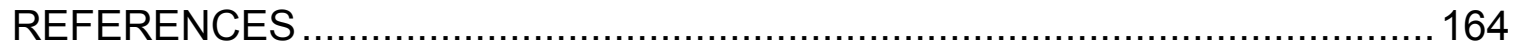

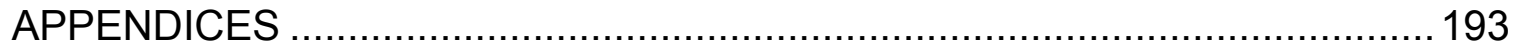

A. Human Subjects Protection Program Office \& Study Site Approval

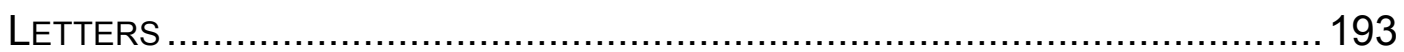

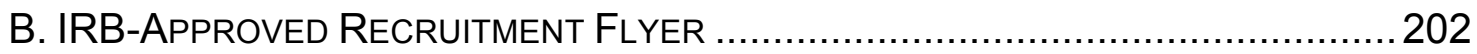

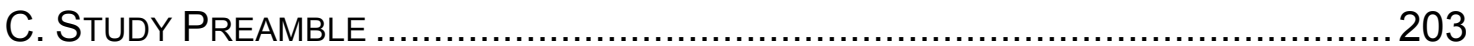

D. ReVISED HEALTHCARE SYSTEM DISTRUST SCALE ....................................205

E. CATALDO LUNG CANCER StIGMA SCALE ...............................................206

F. Modified MarloWe-Crowne Social DesiRABILITy SCALE ..........................209

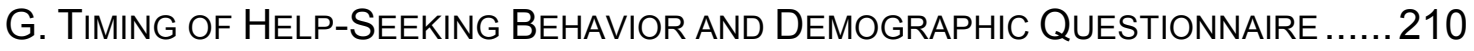

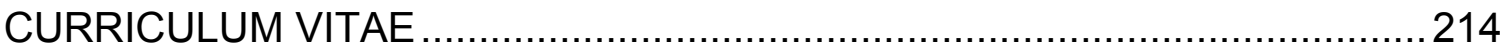




\section{LIST OF TABLES}

TABLE

PAGE

1.1 Non-Small Cell Lung Cancer Staging and TNM Subsets 11

1.2 Five-Year Survival Rates for Non-Small Cell Lung Cancer by Stage. 13

3.1 Measures of Identified Variables 67

3.2 Normality Statistics for Continuous Variables 84

3.3 Screening for Multicollinearity for Continuous Independent Variables 88

4.1 Descriptive Data of Study Participants - Categorical Variables 92

4.2 Descriptive Characteristics of Study Participants - Lung Cancer Stage at Diagnosis and Smoking Status 94

4.3 Prevalence of Symptoms Experienced by Study Participants. 96

4.4 Descriptive Statistics and Reliability of the Revised Healthcare System Distrust Scale and Subscales and the Cataldo Lung Cancer Stigma Scale and Subscales

4.5 Correlations of the Cataldo Lung Cancer Stigma Scale Items with Time to Seek Help 102 
4.6 Differences in the Means Between Categorical Covariates and Continuous

Independent Variables and the Dependent Variable. 113

4.7 Chi-Square Test for Independence Between Covariates (Categorical

Variables) and Categorical Independent Variable (Smoking Status) .......115

4.8 Differences in the Means of Time to Seek Help in Days by Demographic

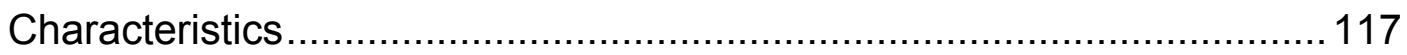

4.9 Summary of Hierarchical Regression Analysis for Variables Predicting

Time to Seek Help in Days (Lg10) in Lung Cancer ................................. 119 


\section{LIST OF FIGURES}

FIGURE

PAGE

1.1 Schematic of Conceptual Model for Understanding Delayed Presentation

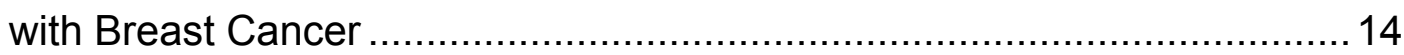

1.2 Schematic of Conceptual Model for Help-Seeking Behavior in Lung

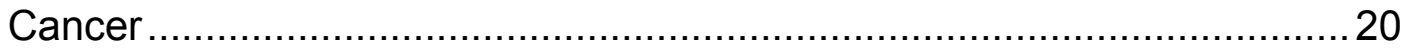

1.3 Schematic of Dissertation Study Conceptual Model ................................28

3.1 Graphic Representation of Normality of Independent Variables ..................85

3.2 Histograms and $Q-Q$ Plots of Time to Seek Help: Raw versus

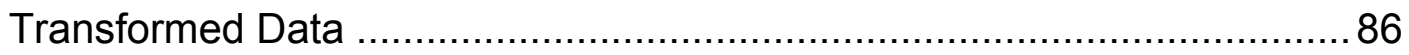




\section{CHAPTER ONE}

\section{BACKGROUND AND SIGNIFICANCE AND CONCEPTUAL MODEL Research Problem}

\section{Background and Significance}

Incidence.

Lung cancer kills more people than any other cancer worldwide (Ferlay et al., 2010; Jemel et al., 2011) with an estimated 1.6 million new diagnoses and an estimated 1.4 million deaths annually (Ferley et al., 2010; Jemel et al., 2011). In the United States it is estimated there will be 228,190 new lung cancer diagnoses and 159,480 lung cancer related deaths (ACS, 2013). This accounts for $14 \%$ of all categories of cancer diagnoses and $27 \%$ of all cancer related deaths respectively. The morbidity and mortality of lung cancer is directly related to its stage at diagnosis (ACS, 2013; Jemel et al., 2011). Only $15 \%$ of lung cancers are detected at a stage amenable to curative resection and the overall five-year survival rate is merely $16 \%$ (ACS, 2013). For the two main types of lung cancer (small cell versus non-small cell), there are striking differences in the survival rates. The American Cancer Society (2013) reports the five-year survival rate for small cell lung cancer is $6 \%$ compared to $17 \%$ for non-small cell lung cancer. Non-small cell lung cancer is the most common type of lung cancer representing 85 to $90 \%$ of lung cancer diagnoses. 
Diagnosis of lung cancer takes various pathways. Lung cancer may be diagnosed in asymptomatic individuals who present for other health concerns; however, some individuals do experience symptoms. Individuals who experience symptoms may perceive the symptoms as alarming and seek help. Others may not perceive the symptoms as alarming and subsequently monitor and selfmanage their symptoms for varying lengths of time (Levealahti, Tishelman, \& Ohlen, 2007; Tod \& Joanne, 2010). When individuals delay seeking help, the probability of a later stage diagnosis increases. Lung cancer is often thought to be asymptomatic until advanced; however, many individuals with early stage lung cancer do have symptoms prior to their diagnosis, but often they do not make the link between what they are experiencing and the potential for lung cancer (Corner, Hopkinson, Fitzsimmons, Barclay, \& Muers, 2005).

Lung cancer kills more people than any other cancer primarily because it is often diagnosed at an advanced stage (Ferley et al., 2010; Jemel et al., 2011; Surveillance, Epidemiology, and End Results (SEER) Program, 2011). The predominance of late stage diagnosis is thought to be related to attributing symptoms to another etiology, an absence of symptoms, or a presence of vague, ambiguous symptoms (Tod \& Joanne, 2010). Although attributing symptoms to another etiology is a prevailing belief, Corner, Hopkinson, Fitzsimmons, Barclay, and Muers (2005) found in their study of 18 lung cancer patients that these individuals did have symptoms prior to their diagnosis regardless of stage at diagnosis; however, they did not connect the symptoms they were experiencing with the potential for lung cancer, challenging the notion that lung cancer is a 
silent disease. This is notable because earlier recognition of lung cancer symptoms combined with earlier help-seeking behavior after an individual becomes aware of a symptom may decrease mortality in lung cancer.

\section{Statement of the problem and significance for nursing.}

While there are lung cancer patients that are asymptomatic when diagnosed, this study was focused on patients who were aware of a symptom. Some people have symptoms suggestive of lung cancer, but delay seeking healthcare. Of these people, some do not recognize that their symptoms suggest lung cancer. On the other hand, some individuals do recognize symptoms, but still choose not to seek healthcare. For those that choose to delay seeking healthcare after recognizing lung cancer symptoms, exploring factors that influence why those individuals chose this response is critical. This research explored healthcare system distrust, lung cancer stigma, and smoking status as factors that may influence the timing to seek healthcare in individuals with lung cancer symptoms. If healthcare system distrust, lung cancer stigma, and/or smoking status are identified as influential variables in the timing of help-seeking behavior, these findings can guide future intervention research.

Delayed help-seeking behavior increases the morbidity and mortality in individuals with lung cancer (Corner, Hopkinson, \& Roffe, 2006; Tod, Craven, \& Allmark, 2008; Tod \& Joanne, 2010). The lack of clear symptoms or attributing symptoms to another etiology coupled with lack of adequate screening mechanisms cause many patients to be diagnosed when their lung cancer has advanced. Advanced stage lung cancer leads to death in the majority of its 
victims with a five-year survival rate of less than $5 \%$ (ACS, 2013). A decrease in the time to diagnosis will decrease mortality rates in lung cancer. When lung cancer is diagnosed at an earlier stage, survival rates are longer (ACS, 2013). However, addressing this issue is multifactorial.

Although the American Cancer Society (2013) recently published screening guidelines for lung cancer, low-dose spiral computed tomography (LDCT) is not a common practice and targets a select population. Unlike the common screening practices that currently reach a broad population for breast and prostate cancers, lung cancer screening is in its initial stages. Earlier recognition and screening by healthcare professionals is a key factor in lung cancer. Increased public health awareness about lung cancer and its symptoms are also important and can lead to increased symptom awareness and individuals potentially seeking help earlier. Earlier recognition of lung cancer symptoms combined with early help-seeking behavior after an individual becomes aware of a symptom will decrease mortality in lung cancer. There is a dearth of research examining timing of help-seeking behaviors in lung cancer. Nursing is a key discipline capable of public health awareness and is in a unique position within the healthcare system to address earlier recognition of lung cancer and promote earlier help-seeking behavior through practice, policy, and research.

\section{Purpose of the Study}

The purpose of this study was to explore the influence of healthcare system distrust, lung cancer stigma, and smoking status on the timing of help- 
seeking behavior in individuals with symptoms suggestive of lung cancer. This was an extension of pilot research on help-seeking behaviors in lung cancer patients in which delayed help-seeking behavior was a prominent theme in the findings. Research supports the notion that healthcare system distrust (Egede \& Ellis, 2008; Katapodi, Pierce, \& Facione, 2010; Shea et al., 2008), lung cancer stigma (Cataldo, Slaughter, Jahan, Pongquan, \& Hwang, 2011; Else-Quest, Loconte, Schiller, \& Hyde, 2009), and smoking status (Stuber, Galea, \& Link, 2008) may influence the timing of help-seeking behavior. Lung cancer stigma (Cataldo et al., 2011; Else-Quest et al., 2009) and smoking status (Stuber et al., 2008) have been examined in a few studies; however, delayed help-seeking behavior was not the primary focus. Healthcare system distrust has not been investigated in a population of lung cancer patients. In addition, healthcare system distrust, lung cancer stigma, and smoking status have never been studied together in a population of lung cancer patients as variables that may potentially influence the timing of help-seeking behavior. This study will also serve as a foundation for future intervention work targeting public health awareness on lung cancer focusing on decreasing the time to diagnosis. The specific aims and their associated hypotheses were:

Aim 1. To explore the influences of healthcare system distrust, lung cancer stigma, and smoking status on the timing of help-seeking behavior in individuals with symptoms suggestive of lung cancer.

H 1.1 Higher scores on healthcare system distrust is positively related to greater time to seek help in lung cancer. 
H 1.2 Higher scores on lung cancer stigma is positively related to greater time to seek help in lung cancer.

H 1.3 Time to seek help for lung cancer will be significantly different for individuals who are currently active smokers compared to former smokers or never smokers.

Aim 2. To examine the bivariate relationships of socioeconomic status, ethnicity, and social desirability as related to healthcare system distrust, lung cancer stigma, smoking status, and timing of help-seeking behavior.

H 2.1 Socioeconomic status will be inversely related to healthcare system distrust, lung cancer stigma, and time to seek help in lung cancer.

H 2.2 Smoking status will be significantly related to socioeconomic status. H 2.3 Ethnicity will be significantly related to healthcare system distrust, lung cancer stigma, smoking status, and time to seek help in lung cancer. H 2.4 Social desirability will be inversely related to healthcare system distrust and lung cancer stigma.

H 2.5 Social desirability will not be significantly related to time to seek help in lung cancer.

Aim 3. To determine the predictive power of the model for timing in helpseeking behavior in lung cancer patients.

H 3.1 Healthcare system distrust, lung cancer stigma, and smoking status will predict greater time to seek help in lung cancer. 


\section{Overall Research Hypothesis}

To address a goal of decreasing the time to diagnosis in lung cancer patients, research that examines the timing of help-seeking behavior in individuals with symptoms consistent with lung cancer is imperative. This research will assist clinicians, researchers, and the public to understand how healthcare system distrust, lung cancer stigma, and smoking status influence the timing of help-seeking behavior during the pre-diagnosis period. The purpose of the study was addressed through testing the following research hypothesis:

Greater healthcare system distrust, higher levels of self-perceived lung cancer stigma, and positive smoking status predict increased time from symptom onset to help-seeking, controlling social desirability, socioeconomic status, and ethnicity.

The next section will discuss an overview of lung cancer, for context, including lung cancer symptoms, staging, and prognosis followed by a discussion of the conceptual model used for the study.

\section{Lung Cancer}

Lung cancer is a carcinogenic process involving two main types: nonsmall cell and small cell lung cancer. Microscopically, lung cancer includes four major histologic categories: squamous cell carcinoma, adenocarcinoma, small cell carcinoma, and large cell carcinoma (Houlihan, 2006). Of these, squamous cell carcinoma, adenocarcinoma, and large cell carcinoma comprise non-small cell lung cancer. Small cell lung cancer is a combined small cell carcinoma. 
Eighty-five to ninety percent of all lung cancers in the United States are non-small cell lung cancer with adenocarcinoma the most common form representing approximately $40 \%$ of all lung cancers (ACS, 2013). The remaining 10 to $15 \%$ is the very aggressive small cell lung cancer that is categorized as either limited or extensive. Limited disease (LD) is confined to one hemithorax and has no pericardial or pleural effusion while extensive disease (ED) represents all other presentations of small cell lung cancer. The biology of lung cancer begins with a single genetic mutation of a bronchial epithelial cell in response to exposure to a carcinogen. There are complex interactions that occur leading to molecular changes and key pathway activation leading to the loss of cellular control. Proliferation of mutant cells then ensues (Houlihan, 2006).

Numerous risk factors for lung cancer have been identified including tobacco smoking, exposure to secondhand smoke, radon and other chemical exposure as well as a history of tuberculosis (ACS, 2013). The American Cancer Society (2013) also notes that genetic susceptibility plays a contributing factor in lung cancer development. Most striking is the fact that 10 to $15 \%$ of people diagnosed with lung cancer have never smoked (Thun et al., 2006). Further, nonsmokers that have been exposed to secondhand smoke reportedly have a 20 to $30 \%$ increased risk of developing lung cancer in their lifetime (Blair \&

Freeman, 2006; Department of Defense, 2010; Thun et al., 2006).

\section{Symptoms of Lung Cancer}

Symptoms are the indicators that lead people to seek healthcare. There is a wide range of symptoms with which lung cancer patients present. The most 
common symptoms in lung cancer are cough, dyspnea, and fatigue (ACS, 2013; Houlihan, 2006). Other regional thoracic manifestations include hemoptysis, wheezing, chest pain, stridor, and hoarseness. Symptoms of extrathoracic involvement include headache, central nervous system involvement, gastrointestinal involvement, jaundice, hepatomegaly, and abdominal pain. In addition, systemic symptoms include weakness, anorexia, cachexia, weight loss, and anemia (ACS, 2013; Houlihan, 2006). If the lung cancer has advanced, the symptoms are related to metastatic disease.

Empirical data suggest that the majority of lung cancer patients present with cough, pain, and fatigue. Three important symptom clusters in lung cancer patients $(N=400)$ have been identified as pain, mood, and respiratory clusters finding cough, fatigue, and pain most prominent (Henoch, Ploner, \& Tishelman, 2009). A smaller study found that $98 \%$ of women $(N=26)$ in an outpatient setting experienced three or more symptoms the day before presenting with complaints that led to their lung cancer diagnosis. Specifically, $80 \%$ of the women reported fatigue and cough in addition to shortness of air, pain, and anorexia (Brown, Cooley, Chernecky, \& Sarna, 2011).

Several studies (Corner et al., 2006; Tod et al., 2008; Tod \& Joanne, 2010) suggest that some people may delay seeking help for lung cancer because the nature of the symptoms may make interpretation difficult. Symptoms may be vague or ambiguous. They may be masked by other comorbid conditions such as emphysema, chronic obstructive pulmonary disease, or other chronic lung conditions. If the individual is a smoker, lung cancer symptoms may simply be 
dismissed as a sequela of smoking. Most importantly, failure to recognize lung cancer symptoms often results in longer time to seek help which can subsequently result in more advanced stage lung cancer at diagnosis and fatal consequences (Mohammed et al., 2011).

\section{Staging and Prognosis of Lung Cancer}

The major indicator of prognosis in all lung cancers is staging (Houlihan, 2006). Luo, Chen, Narsavage, and Ducatman (2012) found advanced cancer stage (IIIB or IV) has independent prognostic significance linked to shorter survival time in non-small cell lung cancer $(N=110)$. While small cell lung cancer is staged as LD or ED, the International System for Staging Lung Cancer uses the TNM classification subsets for non-small cell lung cancer. T represents the primary tumor; $\mathrm{N}$ represents lymph node involvement; and, $\mathrm{M}$ refers to metastatic spread. The primary tumor is divided into four categories (T1 through T4) representing the site, size, and local involvement of the primary tumor. The smaller the number, the smaller the tumor size and local involvement is typically confined. Lymph node involvement comprises three categories: N1 (bronchopulmonary); N2 (ipsilateral mediastinal); and, N3 (contralateral or supraclavicular involvement). The higher numbers are representative of more extensive lymph node involvement. Finally, metastatic staging is either present (M1) or absent (M0). Houlihan (2006) notes, "the size and location of the tumor at the time of diagnosis is tied directly to the ability to achieve cure" (p. 3). Specifically, with non-small cell lung cancer, delay leads to disease progression (Mohammed et al., 2011). 
While staging for small cell lung cancer is categorized as either LD or ED, non-small cell lung cancer staging ranges from Stage 0 to Stage IV. Table 1.1 represents stage grouping and their associated TNM subsets.

Table 1.1

Non-Small Cell Lung Cancer Staging and TNM Subsets

\begin{tabular}{|c|c|}
\hline Stage & TNM Subset \\
\hline 0 & Carcinoma in situ \\
\hline IA & T1 N0 M0 \\
\hline IB & T2 N0 M0 \\
\hline IIA & T1 N1 M0 \\
\hline IIB & T2 N1 M0 \\
& T3 N0 M0 \\
\hline IIIA & T3 N1 M0 \\
& T1 N2 M0 \\
& T2 N2 M0 \\
& T3 N2 M0 \\
\hline IIIB & T4 N0 M0 \\
& T4 N1 M0 \\
& T4 N2 M0 \\
& T1 N3 M0 \\
& T2 N3 M0 \\
& T3 N3 M0 \\
& T4 N3 M0 \\
\hline IV & Any T Any N M1 \\
\hline
\end{tabular}

Note. Adapted from Houlihan, 2006, p. 86; T = primary tumor; $\mathrm{N}=$ lymph node involvement; $\mathrm{M}=$ metastatic spread 
Unlike other cancers such as breast and prostate, there is no routine screening for lung cancer. There have been preliminary results to identify lung cancer early, and most recently, the American Cancer Society (2013) published lung cancer screening guidelines (Wender et al., 2013) resulting from the National Lung Screening Trial (NLST) findings. Research by Aberle et al. (2011) found low-dose spiral computed tomography (LDCT) screening reduces mortality rates from lung cancer. A $20 \%$ decrease in mortality was noted in the LDCT group when compared to the group that received chest radiography. The NLST was a nationwide study that enrolled over 53,000 individuals at high risk for lung cancer from 2002 to 2004 . This finding is promising because detecting lung cancer at an earlier stage decreases the mortality rate. However, these results were only for high-risk individuals and complicated by a number of false positive results (Aberle et al., 2011). Table 1.2 details the five-year survival rates for the stages of non-small cell lung cancer (ACS, 2013), which shows earlier stage diagnosis has the greatest survival rate. 
Table 1.2

Five-Year Survival Rates for Non-Small Cell Lung Cancer by Stage

\begin{tabular}{|c|c|}
\hline Stage & 5-year Survival Rate \\
\hline IA & $49 \%$ \\
\hline IB & $45 \%$ \\
\hline IIA & $30 \%$ \\
\hline IIB & $31 \%$ \\
\hline IIIA & $14 \%$ \\
\hline IIIB & $5 \%$ \\
\hline IV & $1 \%$ \\
\hline
\end{tabular}

Note. ACS, 2013

Because the morbidity and mortality of lung cancer is directly related to its stage at diagnosis (ACS, 2013; Jemal et al., 2011), understanding the timing of help-seeking behavior after a symptom suggestive of lung cancer is discovered is critical. When people do not recognize lung cancer symptoms as suggestive of lung cancer, the disease advances and people die (Mohammed et al., 2011). Although delays in the timing of help-seeking behavior are frequently encountered in various health conditions (Andersen, Vedsted, Olesen, Bro, \& Sondergaard, 2011; Corner et al., 2005; Corner et al., 2006; Gullatte, Hardin, Kinney, Powe, \& Mooney, 2009; Higginson, 2008; Lesneski, 2010; O’Mahoney, Hegarty, \& McCarthy, 2011; Perkins-Porras, Whitehead, Strike, \& Steptoe, 2009; Tod et al., 2008; Tod \& Joanne, 2010), timing is more poignant in the context of lung cancer because advanced stage diagnosis has dismal survival rates 
compared to other types of cancer. Stage IIIB lung cancer has a $5 \%$ five-year survival rate, and Stage IV lung cancer only has a $1 \%$ five-year survival rate (ACS, 2013).

\section{Conceptual Underpinnings}

Delayed help-seeking behavior is common in lung cancer patients (Corner et al., 2005, Tod et al., 2008, Tod \& Joanne, 2010). While there is not a conceptual model specific to delayed help-seeking behavior in lung cancer, Bish, Ramirez, Burgess, and Hunter (2005) developed a model that conceptualizes the phenomenon of delayed help-seeking behavior in women with breast cancer (See Figure 1.1). Bish and colleagues (2005) developed the model for understanding delayed presentation with breast cancer after exploring empirical evidence in the literature that suggested delayed help-seeking behavior was influenced by multiple complex interactions. Specifically, demographic, clinical, cognitive, behavioral, and social variables were suggested as influential on helpseeking behavior in prior studies (Burgess, Ramirez, Richards, \& Love, 1998; Coates et al., 1992; Ramirez et al., 1999).

Figure 1.1. Conceptual Model

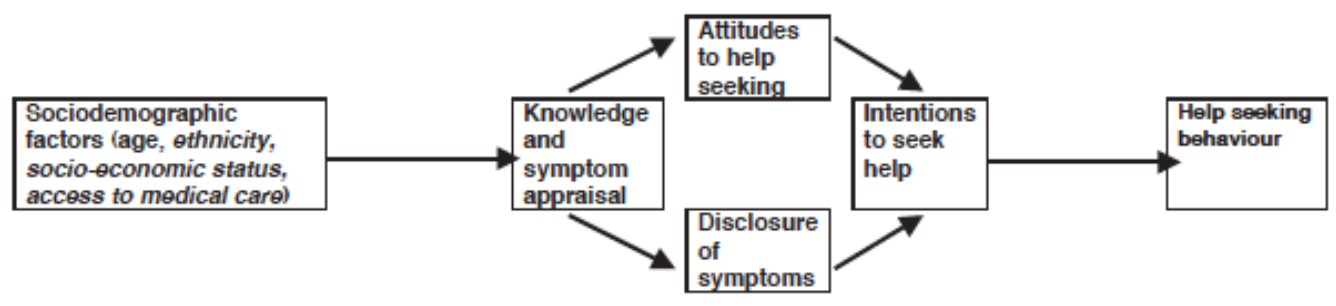

Figure 1.1. Schematic of conceptual model for understanding delayed presentation with breast cancer developed by Bish et al., 2005, Journal of Psychosomatic Research, 58, p. 323 
Bish et al. (2005) identified five primary constructs in their model for understanding delayed presentation with breast cancer leading to the phenomenon of help-seeking behavior: (a) sociodemographic factors, (b) knowledge and symptom appraisal, (c) attitudes to help-seeking, (d) disclosure of symptoms and, (e) intentions to seek help. Bish et al. used components from the theory of planned behavior (Ajzen, 1991), theory of implementation intentions (Gollwitzer, 1993), and self-regulation theory (Leventhal, Nerenz, \& Steele, 1984) to develop this model. Specifically, the constructs of attitudes to help-seeking and intentions to seek help were developed from Ajzen's (1991) and Gollwitzer's (1993) earlier work examining social behavior. Both theoretical models provided support for Bish et al. to use these two constructs in their model. Bish et al. also note the use of Leventhal, Nerenz and Steele's (1984) self-regulation theory to provide the construct of knowledge and symptom appraisal in their model.

The model for understanding delayed presentation with breast cancer (Bish, Ramirez, Burgess, \& Hunter, 2005) proposes that the sociodemographic factors of age, ethnicity, socioeconomic status, and access to medical care influence knowledge and symptom appraisal in breast cancer. Knowledge and symptom appraisal influence both attitude to help-seeking and disclosure of symptoms which in turn influences an individual's intentions to seek help.

The key assumptions of the Bish et al. (2005) model are: (a) intentions to seek help are a result of an individual's attitudes toward help-seeking, (b) intentions to seek help are influenced by an individual's willingness to disclose their symptoms, (c) an individual's attitude toward help-seeking is a manifestation 
of their knowledge level of the health concern and, (d) an individual's positive outcome of help-seeking behavior is a manifestation of a cognitive process related to symptom appraisal (Bish et al., 2005). The authors of this model extended their previous work with help-seeking behavior to specifically address delay as a more prominent component. A search of the literature revealed the model has been used once in a study focused on the development of a psychoeducational intervention for breast cancer awareness (Burgess et al., 2008).

In evaluating the overall strengths and limitations of the model, a limitation is its lack of "predicting" the phenomenon of help-seeking behavior; rather the model functions as an explanation of the phenomenon. However, the explanatory nature of the model can be viewed as one of its strengths because it explores foundational constructs involved in the phenomenon of help-seeking behavior. The following section will explore the individual constructs of the model for understanding delayed presentation with breast cancer.

\section{Sociodemographic Factors}

Age, ethnicity, socioeconomic status, and access to medical care are identified as the sociodemographic factors that influence the help-seeking behavior process. Bish et al. (2005) derived these four sociodemographic factors after reviewing the literature and finding evidence of association between these factors and patient delay. For example, strong correlations are noted between older individuals and patient delay (Ramirez et al., 1999). In addition, empirical evidence supported the inclusion of ethnicity, socioeconomic status, and access to medical care (Burgess et al., 1998; Coates et al., 1992). 


\section{Knowledge and Symptom Appraisal}

Knowledge and symptom appraisal are presented as a unitary concept in this model. This construct can serve as either a facilitating or nonfacilitating variable in the process. These are both mentally-oriented concepts in which the individual is making sense of a change in their health (Bish et al., 2005). Bish and colleagues (2005) note a lesser amount of knowledge of breast cancer symptoms is more likely to lead to a conclusion that the symptom is not serious. Concluding that a symptom is not serious can lead to delayed help-seeking behavior and increased morbidity and mortality with breast cancer. An individual's knowledge level, in turn, affects their attitude toward help-seeking by exposure to knowledge related to symptoms of concern. Therefore, knowledge level is intimately connected to symptom appraisal and in turn, symptom appraisal facilitates increasing an individuals' knowledge level.

\section{Attitudes to Help-Seeking}

Attitude is a way of thinking that is typically reflected in an individual's behavior (Bish et al., 2005). Symptom appraisal influences the construct of attitudes to help-seeking. For example, appraising a symptom as minor or trivial can lead to dismissive attitudes toward help-seeking resulting in delayed diagnosis.

\section{Disclosure of Symptoms}

Bish and colleagues (2005) also note that disclosure of symptoms is important in the phenomenon of help-seeking behavior. Empirical evidence supports disclosure of symptoms to a close friend or family member as influential 
in hastening help-seeking behavior (Burgess et al., 1998; Ramirez et al., 1999). Although not always present, disclosure of symptoms is deemed critical in the help-seeking process when it is present.

\section{Intentions to Seek Help}

The concept of "intention" involves a course of action that results from a decision. Intention is influenced by attitude (Ajzen, 1991; Bish et al., 2005). However, according to Bish et al. (2005), intentions do not always result in actual behavior or the prediction of an expected behavior. This construct was developed from the theory of planned behavior that postulates attitudes lead to intent to carry out a behavior, and in turn, predict behavior. Prediction of behavior is a theory of planned behavior (Ajzen, 1991) postulate whereas there is some disagreement from Gollwitzer (1993) in the theory of implementation intentions. Gollwitzer attempted to bridge the gap between "intentions" and actual behavior by proposing that cognitive influences may explain why some with strong intentions seek help and others with strong intentions do not seek help.

\section{Conceptual Model for Help-Seeking Behavior in Lung Cancer}

The model for delayed presentation with breast cancer (Bish et al., 2005) was adapted for this study to develop a conceptual model for help-seeking behavior in lung cancer (see Figure 1.2). Eight constructs were conceptualized as influential in help-seeking behavior in lung cancer: (a) sociodemographic factors, (b) healthcare system distrust, (c) lung cancer stigma, (d) knowledge of lung cancer, (e) symptom appraisal, (f) attitudes to help-seeking (g) disclosure and, (h) intentions to seek help. Key changes made to the original model by Bish 
et al. (2005) were the addition of healthcare system distrust and lung cancer stigma along with the separation of knowledge and symptom appraisal from one construct into two constructs. The following section will detail the constructs of the model for help-seeking behavior in lung cancer. The constructs will be discussed in order of the model. 


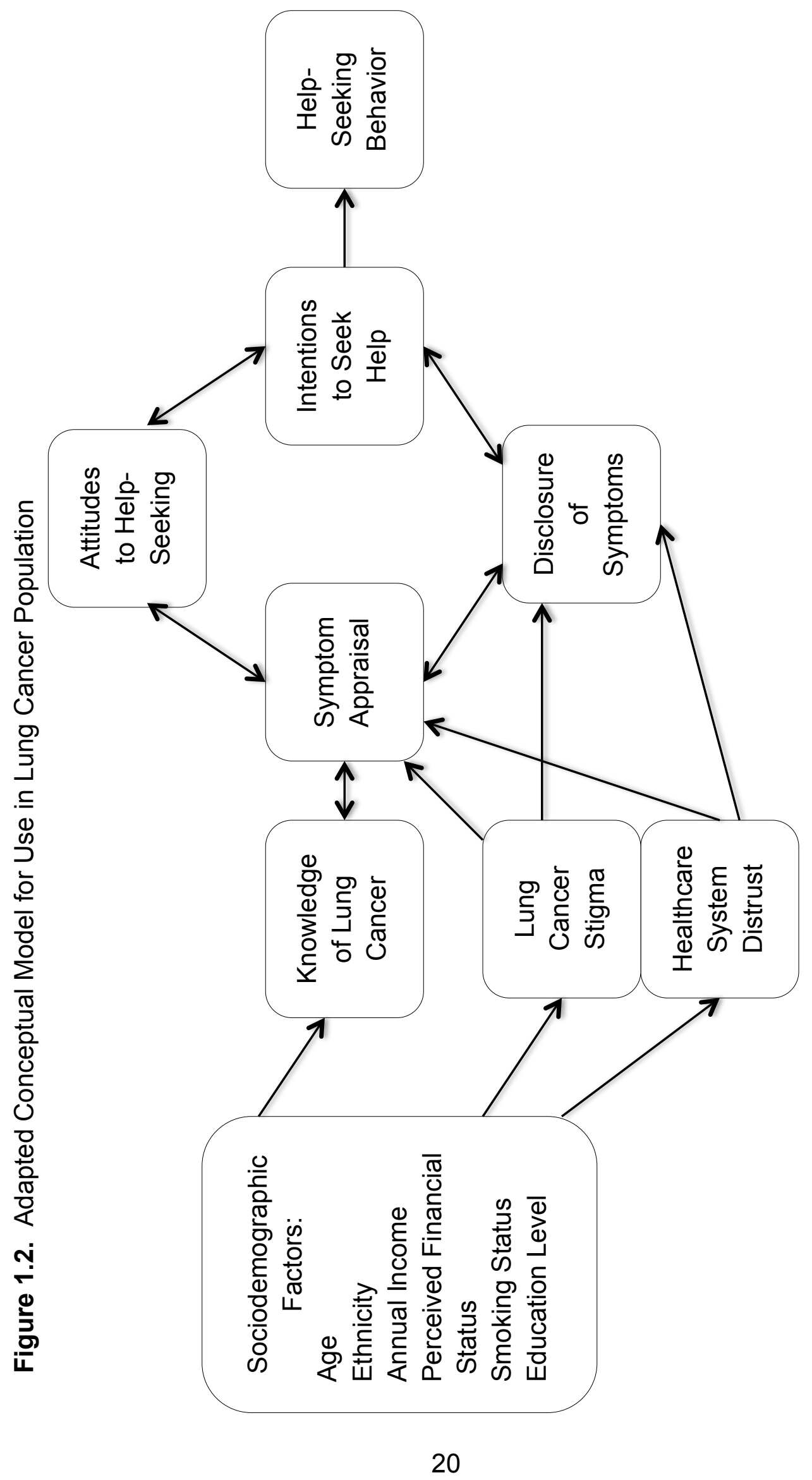

$\frac{\grave{2}}{\frac{0}{0}}$

$\stackrel{\Xi}{\mp}$

든

임

तิ

(

ปั)

ర্ত

몰

.

흐 유

तั

बิ

음

产

పे

का

응 늠

ᄃ 어

는

ฮ Ф

ํํㅇ

ह. .

꼴 을

응 휴

U ํำ

ठㅇ ญ

पㅇ

윰

त

ब $\frac{0}{0}$

을

음

도

(1) क़

这高 


\section{Sociodemographic Factors}

Sociodemographic factors encompass a wide range of variables that potentially influence the time to seek help in symptoms suggestive of lung cancer. Specifically, age, annual income, perceived financial status, access to healthcare, education level, family history of lung cancer, and smoking status are important to include in a model for understanding help-seeking behavior in symptoms suggestive of lung cancer. In breast cancer, older age (Macleod, Mitchell, Burgess, Macdonald, \& Ramirez, 2009; Ramirez et al., 1999), lack of access to healthcare (Burgess et al., 1998; Coates et al., 1992; Koyi, Hillerdal, \& Branden, 2002), minority ethnicity, and lower socioeconomic status (Burgess et al., 1998; Coates et al., 1992) have been associated with delayed help-seeking behavior. Lower socioeconomic status has also been associated with delayed help-seeking behavior in upper gastrointestinal and urologic cancers (Macleod et al., 2009). Lower education level has been associated with patient delay in breast and colorectal cancers (Macleod et al., 2009) while higher education levels have been associated with reduced delayed help-seeking in cervical cancer (Dhamija, Sehgal, Luthra, \& Sehgal, 1993). Family history has been associated with more timely help-seeking behavior in breast cancer patients (Taib, Yip, \& Low, 2011) as well as individuals with prostate cancer (Macleod et al., 2009). Finally, a positive smoking status has been associated with both stigma and delayed helpseeking behavior in individuals with lung cancer secondary to a masking of symptoms or attribution of symptoms to sequela of tobacco smoking (Cataldo, Jahan, \& Pongquan, 2012; Stuber et al., 2008). 


\section{Healthcare System Distrust}

Distrust of the healthcare system has been proposed as a significant barrier to seeking healthcare when symptoms of concern arise (Rose, Peters, Shea, \& Armstrong, 2004). Distrust has a persistent, negative impact on helpseeking behavior (Katapodi et al., 2010). Individuals that discern a symptom of concern but have a high level of distrust of the healthcare system may not be willing to seek help promptly. Therefore, distrust of the healthcare system is a plausible influential factor on the timing of help-seeking behavior in lung cancer.

\section{Lung Cancer Stigma}

Lung cancer stigma is also proposed as influential on help-seeking behavior. The nature of lung cancer frequently involves a sense of being blamed for the disease (Else-Quest et al., 2009). Therefore, lung cancer stigma has been considered a perceived stigma. Van Brakel (2006) described perceived stigma as an anticipation or fear of discrimination with associated awareness of negative attitudes toward a specific condition. Individuals with lung cancer frequently experience a perceived stigma related to their diagnosis of lung cancer regardless of their smoking status (Cataldo et al., 2011). Current smokers, former smokers, and never smokers report a stigmatization from healthcare providers, family members, and friends because lung cancer is so strongly associated with smoking (Cataldo et al., 2012). Therefore, a perceived stigma related to lung cancer is also a plausible influential factor on the time to seek help in lung cancer. 


\section{Knowledge of Lung Cancer}

Lack of knowledge of other cancers such as breast cancer, testicular cancer, and lymphoma has been found to be a barrier to seeking help in a timely manner (Hazewinkel et al., 2009; Howell, Smith, \& Roman, 2008; Mason \& Strauss, 2004b; Sheikh \& Ogden, 1998). A knowledge deficit can in turn significantly influence symptom appraisal, and symptom appraisal that is not interpreted as serious and alarming can lead to delays in seeking help (Howell et al., 2008; Mason \& Strauss, 2004a; Moloczij, McPherson, Smith, \& Kayes, 2008).

The first component of the help-seeking process in lung cancer is perception of a symptom. Knowledge of lung cancer is an important antecedent to the perception of a symptom suggestive of lung cancer. Knowledge of lung cancer (signs, symptoms, and disease process) influences the individual to conclude the symptoms are reflective of a serious process and not dismissible. This process of awareness culminates in appraisal of the symptom which is influenced by knowledge of lung cancer symptoms as lung cancer.

\section{Symptom Appraisal}

Symptom appraisal is a complex concept that is shaped by many things. According to the foundational components of Bish et al's (2005) model, knowledge, beliefs, perceptions of risk, and a personal mental representation of an illness like cancer are vital to accurate symptom appraisal. Somatic perception of a bodily change activates this self-regulatory process (Leventhal et al., 1984). These changes are evaluated by the person against their previous personal experiences either with self or someone close to them as they evaluate 
the importance of the symptom and its associated risk. Unfamiliar symptoms may lead the individual to attribute the symptoms to a less perceived threat or an existing comorbidity. Because lung cancer symptoms can frequently be vague or ambiguous, individuals with lung cancer may make an erroneous self-diagnosis and self manage those symptoms. Many individuals have difficulty identifying symptoms of lung cancer as lung cancer secondary to a deficit of knowledge that directly impacts symptom appraisal. In addition, symptom appraisal has been documented as the critical phase that affects future behavior in many disease processes (Bish et al., 2005; Mason \& Strauss, 2004a; Moloczij et al., 2008; Shaw, Brittain, Tansey, \& Williams, 2008). Symptom appraisal time has been reported as the time period accounting for the majority of total time to seek help in other cancers secondary to attributing symptoms to something benign (Mason \& Strauss, 2004a; Ristvedt \& Trinkhaus, 2005).

\section{Separation of knowledge level and symptom appraisal.}

As mentioned previously, the construct of the original model termed "knowledge and symptom appraisal" can serve as either a facilitating or nonfacilitating variable in the process toward help-seeking behavior (Bish et al., 2005). Although knowledge level is intimately connected to symptom appraisal, symptom appraisal is interpreted as a critical time period that can either facilitate or impede the process toward help-seeking. For this reason, the conceptual model for this study separates these two concepts into distinct components. It could be hypothesized that increased knowledge regarding signs and symptoms of lung cancer increases the likelihood of appraising the symptoms as concerning 
and warranting evaluation. Further, symptom appraisal can positively or negatively influence the individual's attitude toward help-seeking secondary to a prior experience with a similar symptom and/or the healthcare system.

Ultimately, an individual's knowledge and symptom appraisal can influence their propensity toward symptom disclosure (Bish et al., 2005). The Bish et al. (2005) model proposes the importance of symptom appraisal as a key step in the process of help-seeking but notes insufficiency in explaining the entire process in symptoms suggestive of breast cancer. Prompt help-seeking in lung cancer includes not only correct symptom appraisal and correct attribution of level of concern. In addition, prompt help-seeking behavior could be influenced by attitudes to help-seeking, disclosure of symptoms to others, and intention to seek help.

\section{Attitudes to Help-Seeking}

Attitudes to help-seeking are a mentally-oriented concept involving the mental outlook of the individual toward the self-discerned symptom (Bish et al., 2005). There is empirical support in the literature that attitudes to help-seeking influence actual help-seeking behavior. For example, African-American men reported experiences of other individuals with whom they have a close relationship as significant influence on their attitudes to help-seeking both positively and negatively (Griffith, Allen, \& Gunter, 2011). Another study explored the experiences of individuals with non-Hodgkin lymphoma prior to their diagnosis noting thoughts of symptoms as trivial influenced attitudes to seek help (Wall, Glenn, \& Poole, 2011). 


\section{Symptom Disclosure}

An equally important related concept during this stage of the model is disclosure of the symptom. Symptom disclosure is an action-oriented concept. With disclosure of symptoms, the individual must decide to disclose their health concerns to a family member or significant other, friend, or healthcare provider (Bish et al., 2005). For example, a recent study (Griffith et al., 2011) found that individuals who disclosed their symptoms to their spouse sought help for health concerns in a more timely manner compared to those individuals that did not disclose symptoms in a sample of 105 African-American men. The presence of another individual during symptoms of a stroke hastened help-seeking (Moloczij et al., 2008). These examples support the interpretation that this is another point in the model that can be viewed as a facilitating or nonfacilitating variable toward help-seeking behavior.

\section{Intentions to Seek Help}

The final concept prior to the phenomenon of help-seeking behavior is intentions to seek help. As mentioned previously in the description of the original model by Bish and colleagues (2005), the concept of "intention" is a mentallyoriented concept that involves decision-making on the part of the individual regarding a course of action. The individuals' decision manifests itself as intention.

In summary, the phenomenon of "help-seeking" is an action-oriented concept that is influenced by the previously described constructs of the model. Help-seeking behavior in symptoms suggestive of lung cancer may be viewed as 
a progressing from "perceiving a symptom" to "making sense of a symptom" to "deciding to act" to "acting".

Conceptual Model for Help-Seeking Behavior in Lung Cancer and Its Use in this Study

The following section will detail how the conceptual model for help-seeking behavior in lung cancer was used in this study. From the adapted conceptual model (Figure 1.2), the following constructs were measured in this study: (a) ethnicity, (b) annual income, (c) perceived financial status, (d) smoking status, (e) healthcare system distrust, (f) lung cancer stigma, (g) symptom appraisal and, (h) timing of help-seeking behavior. Please see the model as used in this study in Figure 1.3. 
Figure 1.3. Components of Conceptual Model for Timing of Help-Seeking Behavior in Lung Cancer Used for Dissertation Study

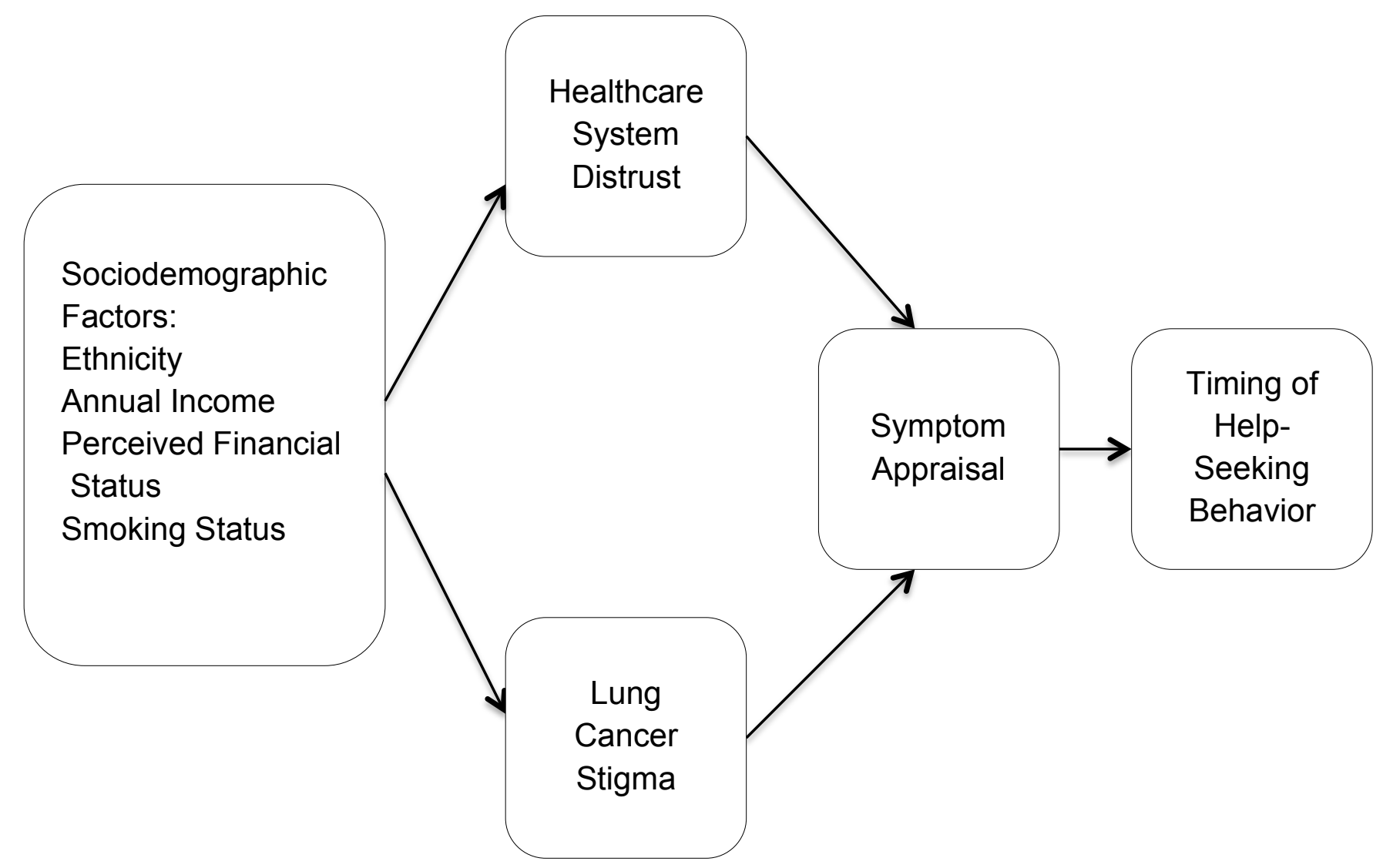

Figure 1.3. Schematic of dissertation study conceptual model 
For this study, multiple sociodemographic variables were collected including age, ethnicity, annual income, perceived financial status, smoking status, insurance status, education level, gender, marital status, and employment status. Specific to the experience of lung cancer, medical characteristics were collected such as symptoms and lung cancer stage at diagnosis. There were three independent variables that were explored as possibilities to influence the time to seek help in lung cancer patients: (a) healthcare system distrust, (b) lung cancer stigma and, (c) smoking status. Healthcare system distrust is a phenomenon that can potentially influence disclosure of symptoms, intentions to seek help, and subsequent help-seeking behavior. Stigma is another phenomenon that can influence an individual's attitudes to help-seeking and subsequent intentions to seek help as well as symptom disclosure. Finally, a positive smoking status has been linked to lung cancer stigma (Stuber et al., 2008) and may subsequently impact symptom disclosure, attitudes to helpseeking, intentions to seek help, and subsequent help-seeking behavior. In addition, a positive smoking status may influence symptom appraisal if the individual attributes their lung cancer symptoms to sequela of smoking. Any or all three variables may be experienced by the individual with lung cancer and may negatively influence the outcome of seeking help with symptoms suggestive of lung cancer.

As mentioned previously, in this study four of the eight constructs of the adapted conceptual model were examined: (a) sociodemographic factors, (b) healthcare system distrust, (c) lung cancer stigma and, (d) symptom appraisal. 
The phenomenon of help-seeking behavior was captured through a semistructured interview assessing symptoms experienced, timing of the first symptom(s), timing of first seeking help for the symptom(s), timing of receiving help, timing of their lung cancer diagnosis, and hindsight symptoms after the diagnosis.

\section{Sociodemographic Factors}

In the original model by Bish et al. (2005), sociodemographic factors included age, ethnicity, socioeconomic status, and access to medical care. A review of the literature (Corner et al., 2006; Levealahti et al., 2007; Tod et al., 2007), as well as findings from the investigator's previous pilot study, supported an expansion of sociodemographic factors in a model focused on understanding help-seeking behavior in symptoms suggestive of lung cancer. Therefore, education level, family history of lung cancer, and smoking status are added to the original models' description of sociodemographic factors.

Certain sociodemographic factors have been found to negatively influence the timing to seek help in symptoms of concern such as lower socioeconomic status (Macleod et al., 2009), financial constraints (Ravenell, Whitaker, \&

Johnson, 2008; Griffith et al., 2011) and inadequate health insurance (Griffith et al., 2011; Rose et al., 2004). However, ethnicity has conflicting results. Frayne, Crawford, McGraw, Smith, and McKinlay (2002) found no significant difference in African-Americans and Caucasians in help-seeking behavior in coronary heart disease but Bird, Woods, and Warren (2009) note African-Americans and Hispanics delay seeking help for acute cardiovascular symptoms when 
compared to their Caucasian counterparts. Although these results were from a population of participants with cardiovascular disease, it is important to include ethnicity in the analysis of this study to understand its potential influence in lung cancer.

In addition, insurance status, gender, marital status, and employment status were measured in this study. These additional sociodemographic items were included to capture a broad picture of the individuals participating in the study. Many sociodemographic factors are not easily amenable to change, but they are factors that are important in identifying the target population for future intervention work (Bish et al., 2005).

\section{Healthcare System Distrust}

Healthcare system distrust is a phenomenon that can have a persistent, negative impact and serve as a significant barrier to seeking help in symptoms of concern (Katapodi et al., 2010; Rose et al., 2004). Higher levels of healthcare system distrust are proposed to have a significant positive relationship with time to seek help in lung cancer.

\section{Lung Cancer Stigma}

Lung cancer stigma is another phenomenon that is proposed as influential on help-seeking behavior. Regardless of smoking status, lung cancer has been associated with a perceived stigma (Cataldo et al., 2011), and this perceived stigma is a plausible influential variable on the timing to seek help in lung cancer. Therefore, higher levels of lung cancer stigma are proposed to have a significant positive relationship with time to seek help in lung cancer. 


\section{Symptom Appraisal}

Symptom appraisal is the initial awareness of a change in health and the individuals' assessment of that change (Bish et al., 2005). Symptom appraisal is a critical component that precedes the individuals' response of disclosure of those symptoms either to a loved one or a healthcare provider or both. However, disclosure of the symptoms can also influence additional appraisal of the change in health. Symptom appraisal was assessed in this study through a semistructured interview with the participant asking for them to recall and describe their first symptom(s). Timing of the symptom(s) was also recorded.

\section{Help-Seeking Behavior}

Finally, help-seeking behavior in this study was examined from a temporal outcome perspective. Timing of help-seeking behavior is the dependent variable of interest. This is the culmination point of all of the mentally-oriented and actionoriented constructs that preceded the behavior of help-seeking. This was assessed in this study by determining a discrete time point as recalled by the participant of seeking help for the symptom that suggested lung cancer.

\section{Conceptual and Operational Definitions of Variables}

The independent variables used in this study are healthcare system distrust, lung cancer stigma, and smoking status. The dependent variable is timing of help-seeking behavior. The covariates are social desirability, socioeconomic status, and ethnicity. Empirical evidence in the literature supports ethnicity (Bird, Woods, \& Warren, 2009; Bish et al., 2005; Burgess et al., 1998; 
Coates et al., 1992) and socioeconomic status (Macleod et al., 2009; Bish et al., 2005; Burgess et al., 1998; Coates et al., 1992) as potential covariates. In addition, due to the sensitive nature of the independent variables, distrust and stigma, social desirability was taken into consideration for this study. Because help-seeking behavior is frequently examined in the literature from the perspective of delayed behavior, patient delay is also included in the following list of definitions.

\section{Timing of Help-Seeking Behavior}

Help-seeking is defined by Cornally and McCarthy (2011) "as a process that begins in response to a problem that cannot be solved or improved alone and involves the active pursuit of and interaction with a third party" (p. 282). This variable will be measured in days as the timing of help-seeking behavior from the first symptom to the first time of seeking help.

\section{Patient Delay}

George T. Pack and James S. Gallo coined the term "patient delay" in 1938 in the context of a breast symptom as "the time elapsed between the onset or discovery of a symptom and the first visit to a physician being greater than three months" (p. 443). This definition has remained the cornerstone definition of more recent work in help-seeking behavior in women with breast cancer (Facione, 2002).

\section{Healthcare System Distrust}

Healthcare system distrust "implies negative beliefs that the trustee will act in ways against the best interest of the truster" (Rose et al., 2004, p. 57). This 
variable was measured as a numeric score on the nine-item Likert-type Revised Healthcare System Distrust Scale. Higher scores equates to higher levels of healthcare system distrust.

\section{Lung Cancer Stigma}

Cataldo, Slaughter, Jahan, Pongquan and Hwang (2011) report that, "stigma in lung cancer is based on the belief that the patient's behavior was the cause of the cancer" (p. E46). This variable was measured as a numeric score on the 31-item Likert-type Cataldo Lung Cancer Stigma Scale. The higher the score is reflective of higher levels of lung cancer stigma.

\section{Smoking Status}

Smoking status refers to the level of smoking behavior by an individual and is defined according to the Centers for Disease Control and Prevention's (2008) Behavioral Risk Factor Surveillance System (BRFSS) as current smoker, former smoker, and never smoker. Smoking status was measured with a demographic question on the Timing of Help-Seeking Behavior and Demographic Questionnaire (THSBDQ).

\section{Social Desirability}

Social desirability is a distortion of a response on a test item in response to what an individual thinks is a socially acceptable answer (Crowne \& Marlowe, 1960). In addition, Kristiansen and Harding (1984) defined social desirability as "the need of subjects to obtain approval by responding in a culturally appropriate and acceptable manner" (p. 385). Social desirability was measured with the 20- 
item dichotomous Modified Marlowe-Crowne Social Desirability Scale (Strahan \& Gerbasi, 1972).

\section{Socioeconomic Status}

Socioeconomic status is a composite measure typically measured by three related indicators of economic status (measured by income), social (measured by education), and work status (measured by occupation) (Adler, 1994; CDC, 2011). Socioeconomic status was measured in this study with demographic questions on the Timing of Help-Seeking Behavior and Demographic Questionnaire (THSBDQ). First, annual income was measured as a self-selected response assigning oneself to an income category. Second, an income adequacy question was included in the THSBDQ assessing the participant's perception of financial status. Education level and occupation were also included in the THSBDQ.

\section{Ethnicity}

Conceptually, ethnicity is defined as a composite of six main features: a common proper name, common ancestry, shared historical memories, elements of a common culture, a link with a homeland, and a sense of solidarity with a population (Baumann, 2004). Ethnicity was assessed with a demographic question on the THSBDQ. Participants were asked to classify their ethnicity as White, Black or African-American, Asian, Native Hawaiian or Other Pacific Islander, American Indian or Alaska Native, or other. If other, they were provided a space to identify their ethnicity. 
In summary, the model for understanding delayed presentation with breast cancer (Bish et al., 2005) was used as a framework to reconceptualize a model for help-seeking behavior in lung cancer (Figure 1.2). This model serves as a foundation for understanding the process of help-seeking behavior in symptoms suggestive of lung cancer. Specifically, this study measured four of the eight constructs of the new model (Figure 1.3): sociodemographic factors, healthcare system distrust, lung cancer stigma, and symptom appraisal as well as the phenomenon of help-seeking behavior captured in this study as the time to seek help in lung cancer.

\section{Summary}

Lung cancer kills more people than any other cancer worldwide (Ferley et al., 2010; Jemel et al., 2011). A primary reason for the large number of deaths is that it often remains undiagnosed until it is in an advanced stage (ACS, 2013). Advanced stage lung cancer is difficult to treat and few individuals survive. Although lung cancer has been portrayed as a "silent disease", the literature reports that symptoms are often present, but individuals may not recognize them as indicative of lung cancer (Tod et al., 2008). Failure to recognize the seriousness of the symptoms may be one reason that individuals delay seeking healthcare; however, other factors may be influential in the help-seeking decisions of individuals. Delayed help-seeking in individuals with lung cancer symptoms may lead to disease progression and subsequent diagnosis at an advanced stage. Understanding the factors that influence the timing of helpseeking behaviors of individuals with symptoms of lung cancer can lead to the 
development of interventions that, when implemented, may decrease the time to diagnosis. A decreased time to diagnosis would decrease patient morbidity from lung cancer. 


\section{CHAPTER TWO}

\section{REVIEW OF THE LITERATURE}

This chapter provides an overview of the state of the science related to help-seeking behavior with particular emphasis on individuals with lung cancer. For contextual purposes, symptoms of lung cancer are reviewed followed by a discussion of help-seeking versus health-seeking behavior. While help-seeking behavior is viewed as a positive phenomenon, the focus in the literature is typically on delayed help-seeking behavior. As such, delayed help-seeking behavior is the primary focus of this review. The discussion focuses on the perspective of individuals with acute cardiovascular events, breast cancer, and lung cancer with the primary emphasis on the latter. It is acknowledged that helpseeking behavior in cardiovascular events and breast cancer is not synonymous with lung cancer. Lung cancer has a different disease trajectory including disease progression and evaluation. However, the majority of studies focused on helpseeking behavior are in the context of acute cardiovascular events or breast cancer.

From patient delay with self-discerning symptoms to provider delay in diagnostic testing and treatment to system delay within the healthcare organizational structure, the phenomenon of delay can have far-reaching 
ramifications on the health of the individual involved. Although there are patient, provider, and system delays in the timing of a lung cancer diagnosis, patient timing to seek help in symptoms suggestive of lung cancer is the focus of this study. Understanding the timing of help-seeking behavior after a symptom is discerned is vital. Because lung cancer stigma (Tod et al., 2008), healthcare system distrust (Armstrong et al., 2008; Gamble, 1997; Katapodi et al., 2010), and positive smoking status (Stuber et al., 2008) are plausible associations with delayed help-seeking behavior as well as the focus of this study, the current state of the science and how they influence help-seeking behavior will be explored. Smoking status will be explored and presented in the section exploring lung cancer stigma.

\section{Lung Cancer Symptoms}

To understand help-seeking behavior in symptoms suggestive of lung cancer, symptoms commonly reported are presented. Initial work exploring symptoms of lung cancer patients at diagnosis (Sarna \& Brecht, 1997) found that people with lung cancer usually experience a combination of symptoms rather than a single symptom. This combination of symptoms is referred to as a symptom cluster (Sarna \& Brecht, 1997). A recent review of the literature by Chen et al. (2011) found inconsistencies regarding symptom clusters in individuals with lung cancer. Studies identifying symptom clusters in lung cancer patients are inconsistent with both the number of symptom clusters specific to this disease as well as the type of symptoms within each symptom cluster. However, common symptoms that have been identified across studies include 
fatigue, loss of appetite, and nausea (Gift, Stommel, Jablonski, \& Given, 2003; Henoch et al., 2009; Sarna \& Brecht, 1997; Wang et al., 2006; Wang, Tsai, Chen, Lin, \& Lin, 2008). Respiratory symptoms were also identified in the symptom clusters such as cough (Sarna \& Brecht, 1997; Wang et al., 2006; Henoch et al., 2009), dyspnea (Henoch et al., 2009; Wang et al., 2006), and shortness of air (Wang et al., 2006). As mentioned previously, the most common symptoms in lung cancer, regardless of presenting in a symptom cluster or not, are cough, dyspnea, and fatigue (ACS, 2013; Houlihan, 2006).

\section{Help-Seeking versus Health-Seeking}

The understanding of patient delay has historically evolved from the context of patient behavior after discernment of a symptom or change in health. Three terms are used interchangeably in the literature to describe the behavioral response to a health concern: health-seeking, care-seeking, and help-seeking. Care-seeking is frequently seen in writings referencing a theoretical framework of seeking care for a symptom (Lauver, 1994). In a recently published concept analysis, Cornally and McCarthy (2011) addressed the difference in terms noting the term health-seeking is most descriptive of health promotion behaviors and not an accurate description of a behavioral response to a problem. In contrast, help-seeking behavior is defined as "a process that begins in response to a problem that cannot be solved or improved alone and involves the active pursuit of and interaction with a third party" (p. 282). The action of help-seeking is viewed as an active process and intentional (Cornally \& McCarthy, 2011). On the other hand, "health-seeking behavior can occur with or without a health problem 
and covers the spectrum from potential to actual health problem" (p. 282). Helpseeking is a reactive process to a problem, as opposed to a proactive process of prevention. As such, help-seeking behavior is part of a broader process of health-seeking behavior (O'Mahoney \& Hegarty, 2009). Using this concept clarification, help-seeking behavior is the focus of this study.

\section{Help-Seeking Behavior}

In an attempt to understand health, researchers began investigating how individuals decided to seek medical evaluation when they experienced a health concern during the 1980s. Researchers focused the lens on socio-psychological factors such as personal beliefs about health and disease (Berkanovic, Telesky, \& Reeder, 1981) and self-esteem (Nadler, 1987). Berkanovic, Telesky, and Reeder (1981) found prior experience with symptoms and their severity correlated with how highly motivated an individual would be to seek help. Individuals with higher levels of self-esteem felt threatened by the need to seek help and delayed help-seeking with a symptom (Nadler, 1987). This finding led other researchers to realize there was a need to understand help-seeking behavior more completely, particularly in cancer (Facione, Dodd, Helzemer, \& Meleis, 1997; Facione \& Giancarlo, 1998; Lauver, 1994; Mor, Masterson-Allen, Goldberg, Guadagnoli, \& Wool, 1990). The notion of help-seeking behavior was initially investigated in people with breast cancer finding the variable with the most profound impact was attribution of symptoms as benign (Facione et al., 1997; Facione \& Giancarlo, 1998). Mistaking symptoms in breast cancer as benign has long had poor outcomes and these findings have been consistent 
throughout (Lam et al., 2008; Molassiotis, Wilson, Brunton, \& Chandler, 2010). Attributing symptoms to conditions of lesser severity or dismissing symptoms completely is not isolated to breast cancer though, but consistent with findings in acute cardiovascular events as well (Moser et al., 2006; Higginson, 2008; Kaur, Lopez, \& Thompson, 2006; Wyatt \& Ratner, 2004).

Overall, the consensus is that help-seeking behavior is influenced by multiple factors in a variety of populations. Although not exhaustive, specific factors either positively or negatively implicated include cost (Lam et al., 2008; Zhou, 2009), denial and fear (Dubayova et al., 2010; Lam et al., 2008; O'Mahoney et al., 2011; Reifenstein, 2007), lack of knowledge (Brink, Karlson, \& Hallberg, 2002; De Nooijer, Lechner, \& De Vries, 2002; Hazewinkel et al., 2009; Kaur et al., 2006; O’Mahoney et al., 2011), symptom appraisal (Ristvedt \& Trinkhaus, 2005), gender (De Nooijer et al., 2002; De Nooijer, Lechner, \& De Vries, 2003), and culture (Harandy et al., 2010; Kaur et al., 2006; Plowden, 2005; Shaikh, Haran, \& Hatcher, 2008). Historically, the focus of help-seeking behavior has shifted from an examination of personal health beliefs (Berkanovic et al., 1981) to an emphasis on understanding the phenomenon of delay, which will be discussed in detail in the next section.

\section{Delayed Help-Seeking Behavior}

Several decades of research have focused on understanding the phenomenon of "patient delay" and the factors that are involved in the time between discernment of a symptom and seeking help for that symptom. The focus of research was on understanding delayed individual help-seeking 
behavior which is frequently considered a reason for individuals being diagnosed with advanced disease (O'Mahoney \& Hegarty, 2009). The concept of delay has long been considered an important issue, particularly in oncology. Delay is most often divided into "patient delay", "provider delay", and "healthcare system delay" (Burgess, Ramirez, Richards, \& Love, 1998; Caplan \& Helzlsouer, 1992) with the latter category referring to the healthcare system. This study focused on patient delay and the review of the literature will focus on this perspective.

Early descriptions of patient delay date back to 1938 with the seminal work of Pack and Gallo (1938) in their research on delay in the treatment of cancer. They coined the term "patient delay" and defined the phenomenon as "the time elapsed between the onset or discovery of a symptom and the first visit to a physician being greater than three months" (Pack \& Gallo, 1938, p. 443). It was not until 1993 when Dr. Noreen Facione published a manuscript asking the question regarding delay versus help-seeking for breast cancer symptoms that research related to delayed help-seeking behavior became prominent in the literature. Facione (1993) performed a meta-analysis of 12 studies on women with breast symptoms identifying one-third of the women delayed three months or longer. This finding was consistent with Pack and Gallo's earlier work with delay. Facione concluded patient and provider delay to be both underresearched and underestimated. She then embarked on a decade of work that formed the foundation for current studies exploring delayed help-seeking behavior (Facione, 1993; Facione, 2002; Facione \& Giancarlo, 1998). 
Patient delay has pointed primarily to sociodemographic and psychological factors as influential in help-seeking behavior in multiple disease processes (Bibb, 2001; Facione, Miaskowski, Dodd, \& Paul, 2002; Friedman et al., 2006; Hazewinkel et al., 2009; Ristvedt \& Trinkhaus, 2005). Although the relationship between delayed help-seeking behavior and survival is not linear, delay is attributed as a variable in poor patient outcomes reflected in the survival rates of breast cancer (Burgess et al., 2009; Gould, Fitzgerald, Fergus, Clemons, \& Baig, 2010; Linsell et al., 2009) and patients experiencing acute cardiovascular events (Altice \& Madigan, 2011; Dracup et al., 1995; Dracup et al., 2003; FoxWasylyshyn, El-Masri, \& Artinian, 2010; Fukuoka et al., 2007; Fukuoka et al., 2010; Higginson, 2008; Kaur et al., 2006; Khraim, Scherer, Dorn, \& Carey, 2009; Lesneski, 2010; Perkins-Porras et al., 2009).

In the context of breast cancer, help-seeking behavior has been defined as the time elapsed from the first symptom discovered by the woman to the time point that she first seeks an evaluation of her symptoms (Facione, 2002). Ultimately, across the spectrum of cancer, it is key to understand that delay is a significant factor associated with later stage cancer diagnosis (Corner et al., 2005; Corner et al., 2006; Facione \& Facione, 2006; Gullatte, 2006; Gullatte et al., 2009; Gullatte, Phillips, \& Gibson, 2006; Tod et al., 2008). Thus, late stage diagnosis directly impacts morbidity and mortality. Accordingly, the discussion of patient delay in help-seeking behavior will focus on individuals with acute cardiovascular events, breast cancer, and lung cancer in the next three sections of this chapter. 


\section{Delay in Cardiovascular Symptoms}

As one of the leading causes of death in the United States (Minino, Murphy, Xu, \& Kochanek, 2011), coronary heart disease has been the focus of research studies for decades. The acute nature of acute myocardial infarction and acute coronary syndrome make delays between symptom onset and hospital presentation vital to understand as well as address. Dracup and Moser (1991) first examined help-seeking behavior among individuals with signs and symptoms of acute myocardial infarction in the early 1990s. Their pivotal research documented mean delay times ranging from 4.6 to 24 hours (Dracup \& Moser, 1991) finding less than 30 of these minutes representative of transportation time. People were likely to delay seeking help because they did not attribute the symptoms as emergent and cardiac in nature (Ashton, 1999; Burnett, Blumenthal, Mark, Leimberger, \& Califf, 1995; Dracup \& Moser, 1991; Meischke et al., 2000). In fact, many individuals reported waiting to see if the symptoms would resolve in cases of delayed help-seeking behavior in acute myocardial infarction (Moser et al., 2006). Other factors associated with delay in acute cardiovascular events were ethnicity (Zerwic, Hwang, \& Tucco, 2007), female gender, unmarried status, and lower level of educational attainment (Dracup \& Moser, 1991).

Gender differences in acute cardiovascular presentations of women have been found and may help explain an aspect of delayed help-seeking behavior in acute cardiovascular events (Bird et al., 2009; McSweeney, Lefler, Fischer, Naylor, \& Evans, 2007; Sjostrom-Strand \& Fridlund, 2008). Since the initial 
research of the 1990s, the most consistent theme remains non-attribution of symptoms as emergent and cardiac in nature (Fox-Wasylyshyn et al., 2010; Lesneski, 2010; Perkins-Porras et al., 2009). Although public health campaigns of the 1990s and 2000s highlight the importance of emergency hospital care for acute cardiovascular events (Bandura, 2004; Green \& Kreuter, 1990), people continue to fail to connect the importance and severity of the symptoms (Carney, Fitzsimons, \& Dempster, 2002; Fox-Wasylyshyn et al.; Fukuoka et al., 2007; Harralson, 2007; Lesneski; Lovlien, Schei, \& Hole, 2007; McSweeney et al., 2007; Perkins-Porras et al., 2009; Quinn, 2005).

A number of demographic characteristics have been identified as having a greater influence on help-seeking behavior, but some offer conflicting results. With age, for example, while Harralson (2007) found younger age (less than 65 years) were more likely to delay, Tullmann, Haugh, Dracup, and Bourguignon (2007) and Perkins-Porras, Whitehead, Strike, and Steptoe (2009) found older individuals (greater than 65 years) more likely to delay. While there is conflicting evidence supporting a specific age-related demographic element of people more likely to delay, non-attribution of symptoms as emergent and cardiac in nature remains the overarching theme (Fox-Wasylyshyn et al., 2010; Lesneski, 2010; Perkins-Porras et al., 2009).

Until recently, little was known about ethnic differences in help-seeking behavior in acute cardiovascular events because the majority of studies that examined delay in acute cardiovascular symptoms were of middle- to upperclass socioeconomic background Caucasian males (Lee, 1997). More recent 
research explored delay in African-American individuals with acute myocardial infarction (Banks \& Dracup, 2006). The median prehospital delay in a sample of African-American men and women was significantly longer (4.25 hours) than a similar sample of Caucasian patients (2.3 hours). Prolonged delay in acute myocardial infarction was found in single, widowed, or divorced AfricanAmericans, insured African-Americans, and African-Americans with diabetes (Banks \& Dracup, 2006). Banks and Dracup (2006) note their finding of insured individuals delaying longer was unexpected noting the contrary was the finding in a large 2002 study by Gibler et al. (2002).

\section{Delay in Breast Cancer}

Almost simultaneously as the exploration of delayed help-seeking behavior in cardiovascular events began, researchers of help-seeking behavior started to report this phenomenon in breast cancer. This work became foundational to studies investigating help-seeking behavior (Burgess et al., 1998; Caplan, 1995; Caplan, Helzlsouer, Shapiro, Wesley, \& Edwards, 1996; Facione, 1993). Non-attribution of symptoms as alarming is a major factor for delayed help-seeking behavior in breast cancer. Other factors that emerged include nonlump initial breast symptoms and non-disclosure of symptoms to others.

The evidence regarding the relationship between self-discerned breast symptoms and delayed help-seeking behavior have remained consistent with the earlier studies of the 1990s linking non-recognition of symptoms, lack of knowledge regarding symptoms, non-lump breast symptoms, and non-disclosure of symptoms to others with propensity to delay (Gould et al., 2010; Taib et al., 
2011). However, many researchers have started to explore the influence of various demographic factors on delay. Minority ethnic groups in England were associated with delayed help-seeking behavior in various cancer symptoms including breast cancer (Waller et al., 2009). Similarly, in the United States, African-American ethnicity and lower socioeconomic status have been correlated in a few studies with delayed help-seeking behavior in breast cancer patients (Bibb, 2001; Facione et al., 2002; Friedman et al., 2006) suggesting the importance of exploring both demographic factors as potential influential variables in the timing to seek help.

Overall, the consensus is that delay most often results from nonrecognition of symptoms, which echoes the findings in the cardiovascular literature (Burnett et al., 1995; Carney et al., 2002; Fox-Wasylyshyn et al., 2010; Lesneski, 2010; Lovlien et al., 2007; Perkins-Porras et al., 2009; Quinn, 2005). Ultimately, delayed help-seeking behavior in breast cancer patients is multifactorial. While typically breast cancer is not as acute an event as acute myocardial infarction or acute coronary syndrome, the need to connect symptoms that are suggestive of breast cancer as alarming prevails (Gould et al., 2010; Lesneski; Perkins-Porras et al.; Taib et al., 2011).

\section{Delay in Lung Cancer}

While there has been considerable study into the phenomenon of delayed help-seeking behavior in breast cancer patients and acute cardiovascular events, there is a dearth of literature on delayed help-seeking behavior in lung cancer patients. Of the studies identified dating back to 1997, five discuss delay in terms 
of the clinical and surgical management impact (Burmeister et al., 2010; Christensen, Harvald, Jendresen, Aggresrup, \& Petterson, 1997; Jensen, Mainz, \& Overgaard, 2002; Koyi et al., 2002; Salomaa, Sallinen, Hiekkanen, \& Liippo, 2005); one study discusses common signs and symptoms associated with the initial evaluation of lung cancer patients (Spiro, Group, \& Colice, 2007); and, one study examines the characteristics of missed opportunities in the lung cancer diagnosis (Singh et al., 2010). The relevant literature that attempts to explain patient delayed help-seeking behavior in lung cancer is isolated to five studies detailed in six published articles (Corner et al., 2005; Corner et al., 2006; Leprieur et al., 2012; Lovgren et al., 2008; Tod et al., 2008; Tod \& Joanne, 2010). Interestingly, to date, there are no studies that have been conducted in the United States. The relevant literature is isolated geographically to Europe.

As mentioned previously, there are several symptoms suggestive of lung cancer. Until relatively recent years, little was known about symptom difference in early versus late stage diagnosis. Corner et al. (2005) explored the inevitability of late stage diagnosis in lung cancer by examining the trajectory toward diagnosis with a group of recently diagnosed individuals. The researchers examined whether symptom history and pathway toward the diagnosis differed for early stage lung cancer compared to those individuals initially diagnosed at advanced stages. While the first change of health status was generally characterized by one or two symptoms, the number of symptoms at diagnosis was markedly higher (Corner et al., 2005). Regardless of their stage at diagnosis, all study participants recalled having symptoms for many months prior to being diagnosed 
with lung cancer (Corner et al., 2005). Analysis was also done of the key time intervals prior to the diagnosis (Corner et al., 2006). These time intervals included the first persistent change in health, the health change or symptom that prompted help-seeking, and the visit to the doctor that began the diagnostic process (Corner et al., 2006). They concluded that the greatest period of delay in the lung cancer diagnosis process lies in the time before one seeks help and the pathway to the lung cancer diagnosis is remarkably similar regardless of ultimate stage at initial diagnosis (Corner et al., 2005).

Lovgren et al. (2008) investigated time spans from the first symptoms of lung cancer to treatment finding the five most commonly reported initial symptoms to be cough, dyspnea, weight loss, fatigue, and thoracic pain $(N=$ 314) for both men and women. The mean and standard deviation of the time span from first symptom to first visit in the healthcare system was $53+71$ confirming suspected individual delays. Little difference was found between gender and their experience of first symptoms or response to those symptoms. However, they did note a slightly prolonged delay in individuals older than 74 years of age.

A recent study explored delay in elderly individuals with lung cancer between the initial symptoms, diagnosis, and onset of specific treatment (Leprieur et al., 2012). While Lovgren et al. (2008) found longer delays in the elderly individuals in their study, Leprieur et al. (2012) found elderly lung cancer patients delayed a similar time span as younger lung cancer patients (median 1.6 months). In addition, there were no identified differences in the initial symptoms 
of elderly patients compared to younger lung cancer patients $(N=193)$ in their study.

More recent studies have supported the notion that attributing lung cancer symptoms to other conditions (Tod et al., 2008) as well as poor knowledge of lung cancer symptoms (Tod \& Joanne, 2010) are key in delayed help-seeking behavior regardless of demographic factors. Researchers have built upon the findings of Corner and colleagues $(2005,2006)$ to identify factors that influence delay in reporting symptoms of lung cancer (Tod et al, 2008; Tod \& Joanne, 2010). Tod, Craven, and Allmark (2008) conducted a qualitative analysis with 18 participants diagnosed with lung cancer within the previous six months. This study explored factors that influenced delay in reporting lung cancer symptoms. Tod et al. found there to be large variations in symptoms making it difficult to identify a clear symptom profile. A lack of both knowledge and awareness of lung cancer symptoms was apparent. Analysis revealed the participants experienced blame and stigma, fatalistic fears related to the diagnosis, and cultural issues impacted help-seeking behavior (Tod et al., 2008). Another key finding was families as positive key facilitators both in noticing symptoms as well as encouraging the individual to seek help. This association is consistent with helpseeking behavior in acute cardiovascular events (Lesneski, 2010; Zegrean, FoxWasylyshyn, \& El-Masri, 2009) and the breast cancer (Gould et al., 2010; Gullatte et al., 2009; Taib et al., 2011) literature. Support for the importance of sharing symptom knowledge or the witness of symptoms as one reason people hasten help-seeking was found. 
Although researchers have identified the absence of tangible symptoms as a barrier to prompt diagnosis of lung cancer (Tod \& Joanne, 2010), more critical are the symptoms, in hindsight, that could have led to a more timely diagnosis (Spiro et al., 2007). Lung cancer is diagnosed in individuals who many times recall weeks or months of symptoms when diagnosed that they did not originally recognize as alarming (Corner et al., 2005; Tod \& Joanne, 2010).

Tod and Joanne (2010) explored factors influencing delay in reporting symptoms of lung cancer. Healthcare professionals identified factors that contributed to patients' delay in seeking healthcare. The tendency of patients to attribute symptoms to other meanings, individual self-management, and fear of blame and stigma from healthcare providers was deemed the most critical issues that influenced help-seeking behavior in lung cancer (Tod \& Joanne, 2010).

These findings were used to guide development of a tool for use by clinicians to decrease delay in reporting lung cancer symptoms.

In summary, it has been found that help-seeking behavior for a variety of health conditions is affected mainly by a person's assessment of the symptom (Corner et al., 2005; Corner et al., 2006; Lesneski, 2010; Taib et al., 2011; Tod et al., 2008; Zegrean et al., 2009). In cancer, individuals often think symptoms are benign though they actually may be indicative of cancer (Moody, Muers, \& Forman, 2004). Other factors have been found to be influential in help-seeking behavior including cost, gender, culture, denial, and fear (Brink et al., 2002; De Nooijer et al., 2002; De Nooijer et al., 2003; Kaur et al., 2006; Lam et al., 2008; O'Mahoney et al., 2011; Plowden, 2005; Reifenstein, 2007; Shaikh et al., 2008; 
Zhou, 2009). The most prominent adverse outcome of all of these factors in lung cancer is delayed presentation for healthcare (Corner et al., 2005; Corner et al., 2006; Tod et al., 2008; Tod \& Joanne, 2010). In lung cancer patients, an inability to recognize its symptoms and resulting delayed presentation increases the chance of late stage at diagnosis, which results in decreased survival rates. The next section will discuss two variables that have the potential to influence helpseeking behavior. Lung cancer stigma will be discussed first followed by an examination of healthcare system distrust. Smoking status will be discussed within the context of stigma. This section will be followed by a discussion of social desirability and potential implications in this study.

\section{Stigma}

Stigma is frequently cited as a phenomenon that negatively impacts individuals in various ways. Lebel and Devins (2008) define stigma as "a social process, experienced or anticipated, characterized by exclusion, rejection, blame or devaluation that results from experience, perception or reasonable anticipation of an adverse social judgment regarding a person or group" (p. 717). Most attempts at unraveling the dimensions of stigma give reference to Goffman's theory from his seminal 1963 essay entitled Stigma: Notes on the Management of a Spoiled Identity based upon attribution theory. This theory notes that one's actions or inactions may not only be responsible for the stigmatized disease process the individual is experiencing but the very stigma that is experienced as well (Lebel \& Devins, 2008). Goffman (1963) defines stigma as "an attribute that links a person to an undesirable stereotype, leading other people to reduce the 
bearer from a whole and usual person to a tainted, discounted one" (p. 11).

Further, Crocker, Major and Steele (1998) note social stigma originates from an attribute conveying a social identity that has been devalued.

Stigma has long been associated with illnesses that were either poorly understood, poorly defined, lacked effective treatment, and frankly invoked fear within a population (Lebel \& Devins, 2008; Stutterheim et al., 2011; Weiss \& Ramakrishna, 2006). For example, there is a large body of literature on HIVIAIDS related stigma. Since the beginning of the AIDS epidemic, HIVIAIDS patients have been stigmatized (Greene \& Banerjee, 2008). In fact, the word "AIDS" can elicit fear, revulsion, and pity. They found negative attitudes toward homosexuality, high levels of religiosity, and high authoritarian views were related to negative attitudes toward people with AIDS. Lekas, Siegel, and Leider (2011) note people with HIVIAIDS are subjected to multiple layers of stigma primarily attributed to their membership in a perceived disenfranchised group, such as sexual minorities and injection drug users, and may be a self-inflicted disease because of their group membership. In addition, HIVIAIDS related stigma is reportedly correlated to more psychological distress, lower self-esteem, and less social support (Stutterheim et al., 2011).

\section{Lung Cancer Stigma and Smoking Status}

The stigma associated with lung cancer is one of tobacco use blame and self-blame of the individual's own behavior (Bell, Salmon, Bowers, Bell, \& McCullough, 2010; Cataldo et al., 2011; Chapple, Ziebland, \& McPherson, 2004; Else-Quest et al., 2009). Clearly, the high risk of lung cancer development with 
tobacco use has resulted in a stigma surrounding the disease (Lebel \& Devins, 2008). In fact, because lung cancer is often associated with tobacco smoking, lung cancer can invoke similar attribution of blame such as that seen with HIVIAIDS (Marlow, Waller, \& Wardle, 2010; Wolitski, Pals, Kidder, CourtenayQuirk, \& Holtgrave, 2009). This is why delineating smoking status in a study on the timing of help-seeking behavior is important. Stuber, Galea and Link (2008) note the association of smoking status has negative consequence for individuals with lung cancer. However, Cataldo, Jahan, and Pongquan (2012) note whether or not an individual smokes, stigmatization from healthcare providers, friends, and family members is perceived because the disease is strongly associated with smoking. The social stigma of smoking is pronounced leading to negative attitudes held by both non-smokers and smokers (Raleigh, 2010). Lung cancer stigma can, in turn, affect social interactions with health care providers, family, and friends.

Self-blame is linked to poor psychological adjustment in lung cancer patients reinforcing a vicious self-degrading cycle (Else-Quest et al., 2009). Although conversations about stigma are not new, examination of lung cancer stigma is a relatively novel focus appearing in the literature within the past decade. The understanding of lung cancer stigma has historically evolved from the pivotal work of Chapple, Ziebland, and McPherson (2004). They explored the perceptions and experiences of stigma with lung cancer patients. Lung cancer's association with smoking, perceptions of self-infliction, and high death rates has influenced the stigma attached to the disease process (Chapple et al., 2004). 
Therefore, stigma attached to lung cancer is both a "felt stigma" and "enacted stigma". This broad perception of stigma can greatly affect social interaction once a diagnosis is suspected or confirmed. Further, self-image may be detrimentally affected especially if the individual is a smoker for fear that they may be denied treatment (Chapple et al., 2004).

Historically, the understanding of cancer-related stigma has evolved from a fear of suffering and death (Muzzin, Anderson, Figueredo, \& Gudelis, 1994; Stahly, 1989) to encompass a perception of lung cancer as shaped by tobacco abuse and the disease (Chapple et al., 2004). More recent studies support the earlier conception of lung cancer stigma offering a conceptual link between the disease, internalization of stigma, and a resulting self-blame (Else-Quest et al., 2009). Else-Quest, LoConte, Schiller, and Hyde (2009) studied perceived stigma, self-blame, and adjustment in breast, lung, and prostate cancer patients. The researchers found that perceived stigma and self-blame was associated with a poorer psychological adjustment in their sample. Further, they found that selfblame mediated the link between perceived stigma and psychological adjustment. Lung cancer patients were more likely to report higher self-blame, poorer self-esteem, and higher mental maladjustment than breast or prostate cancer patients (Else-Quest et al., 2009). These findings connect back to the earlier findings of the "shame and blame" experience of individuals with lung cancer in the study by Chapple et al. (2004).

The findings from the literature suggest blame and stigma are influential in the timing of help-seeking behavior in lung cancer patients (Tod et al., 2008). 
Specifically, Tod et al. (2008) found an expectation by patients of experiencing blame and stigma if diagnosed with lung cancer. Recent work by Cataldo et al. (2012) also supports these findings. Never smokers and former smokers frequently delay reporting symptoms because of an expectation to be blamed for their illness (Tod et al., 2008). Clearly, this is a potential influence in the timing of help-seeking behavior in lung cancer patients.

\section{Social Desirability}

A key related variable to stigma is the phenomenon of social desirability. A classic definition of social desirability dates back to the work of Douglas Crowne and David Marlowe (1960) in their psychometric testing of participants responding in culturally sanctioned ways in psychopathology studies. Although not the first psychometric measure developed, Crowne and Marlowe wanted to develop scale items that had minimal pathological implications if the respondent answered in the affirmative or negative. Kristiansen and Harding (1984) later defined social desirability as the "need of subjects to obtain approval by responding in a culturally appropriate and acceptable manner" (p. 385). Although social desirability has not been specifically documented in the lung cancer literature, recent studies have supported social desirability bias in HIV-related behavioral surveys (Lowndes et al, 2012) and mental health stigma questions (Henderson, Evans-Lacko, Flach, \& Thornicroft, 2012). Lung cancer is frequently associated with smoking status as well as stigma (Cataldo et al., 2011; ElseQuest et al., 2009; Stuber et al., 2008) and social desirability is a plausible covariate. 


\section{Healthcare System Distrust}

Another phenomenon frequently referenced as a variable that impacts an individual's relationship with the healthcare system is distrust. Much of the early work regarding trust and medicine has focused contextually on trust in one's personal physician concerned primarily with patient satisfaction (Thom, Kravitz, Bell, Krupat, \& Azari, 2002; Thom, Ribisl, Stewart, \& Luke, 1999). However, "healthcare system distrust has emerged as a related but conceptually distinct aspect of trust" (Schenker, White, Asch, \& Kahn, 2012, p. 4). Trust is based upon the perception that the entrusted is capable (technical competence) and the perception that the entrusted wants to do what is needed (values congruence) supporting a multidimensionality to the concept of distrust (Shea et al., 2008).

There is a great deal of literature on African-American distrust of the healthcare system. Veronica Northington Gamble's pivotal essay in 1997 entitled Under the Shadow of Tuskegee: African Americans and Health Care has shaped subsequent literature on the topic. By providing a context that acknowledges the injustices of the Tuskegee Syphilis Study but broadens the perspective to include prior and subsequent prejudice, inequalities and discrimination, a venue has been created for dialogue concerning healthcare system distrust. Gamble (1997) reports "there is a collective memory among African Americans about their exploitation by the medical establishment" (p. 1775). This "collective memory" contributes to the distrust of varied societal institutions and is proposed as an implication in African-American delay in seeking healthcare within this system. 
African-American distrust of the healthcare system has been documented in many areas. Researchers have found African-American distrust in organ donation (Russell, Robinson, Thompson, Perryman, \& Arriola, 2012), preventive screenings (Katapodi et al., 2010), physicians (Armstrong, Ravenell, McMurphy, \& Putt, 2007; Wasserman, Flannery, \& Clair, 2007), and participation in research studies (Corbie-Smith, Thomas, \& St. George, 2002; Corbie-Smith, Thomas, Williams, \& Moody-Ayers, 1999). Researchers have also documented distrust by African-Americans in the healthcare system overall (Armstrong et al., 2007; Dovidio et al., 2008; Katapodi et al., 2010; Kennedy, Mathis, \& Woods, 2007; Wasserman et al., 2007). However, healthcare system distrust is not isolated to the African-American community (Egede \& Ellis, 2008). It is important to recognize distrust in healthcare can occur in diverse contexts and populations including the elderly (Mascarenhas et al., 2006; Yang \& Matthews, 2012), among HIV-positive individuals (Cunningham, Sohler, Korin, Gao, \& Anastos, 2007), women in Appalachia (McAlearney et al., 2012), in an intensive care unit (Schenker et al., 2012), and in the context of genetic testing (Armstrong et al., 2012). Healthcare system distrust is a complex, multidimensional phenomenon that is contextualized in many venues. Although the context is broad, key examples from recent literature will be highlighted below.

Yang, Matthews, and Hillemeier (2011) explored healthcare system distrust as a barrier to breast and cervical cancer screening. Regardless of ethnicity, a statistically significant level of distrust among women was found. Consistent with these findings, McAlearney et al. (2012) explored distrust among 
Appalachian women regarding cervical cancer screening. Findings from this qualitative exploration documented a level of healthcare system distrust that suggested a lack of patient-centered communication and perception of poor quality care fostered the distrust. Thus, healthcare system distrust not only influences the timing of help-seeking behavior when one has a concern, but can greatly impact preventive healthcare as well.

While researchers have yet to specifically address healthcare system distrust and lung cancer, distrust of the healthcare system may be an important barrier to seeking help when symptoms are discerned. People who distrust the healthcare system are more likely to delay help-seeking within that system which has been documented in the breast cancer literature (Katapodi et al., 2010). Distrust has an invasive, persistent negative impact on the timing of help-seeking behavior. "One of the most fundamental characteristics of trust is that it is fragile; it is created rather slowly, while it can be destroyed instantly by a single act of betrayal" (Katapodi et al., 2010, p. 976). Researchers have produced a small body of literature that has opened the dialogue to the measurement of healthcare system distrust (Egede \& Ellis, 2008; Rose et al., 2004; Shea et al., 2008).

Examination of healthcare system distrust has evolved from a focus on why African-Americans frequently decline to participate in research studies (Corbie-Smith et al., 2002; Corbie-Smith et al., 1999) to a beginning dialogue on the relationship of healthcare system distrust and its impact on healthcare utilization by all. Hesitation to use the healthcare system has been implicated in advanced stage at diagnosis both in breast cancer (Friedman et al., 2006; Gould 
et al., 2010; Taib et al., 2011) and lung cancer (Corner et al., 2005; Corner et al., 2006; Tod et al., 2008; Tod \& Joanne, 2010). These findings support the importance of understanding the timing of help-seeking behavior in lung cancer patients more completely.

\section{Literature Review Summary}

In summary, researchers have frequently focused on help-seeking behavior from the perspective of delayed time to seek help. The most consistent finding in all disease processes was a failure to recognize the symptoms as related to the disease process, and more importantly, as alarming. Healthcare system distrust and lung cancer stigma are both variables that can influence the timing of help-seeking behavior. Lung cancer stigma is a perceived stigma resulting from behavioral self-blame and blame of tobacco use (Cataldo et al., 2011; Chapple et al., 2004; Else-Quest et al., 2009). This self-blame reinforces a vicious cycle that can impede the pathway to seek help for the symptom(s). As for healthcare system distrust, the etiology remains poorly understood. At first glance, there are many published reports of African-American distrust of the healthcare system that have been linked to prior atrocities related to healthcare research. However, other researchers on healthcare system distrust note this variable is not isolated to the African-American community supporting a multifactorial etiology. Healthcare system distrust research is at a nascent point and future endeavors may shed more light on the complexities of this variable. Ultimately, lung cancer stigma and healthcare system distrust have the potential to influence the timing to seek help with a lung cancer symptom 


\section{CHAPTER THREE \\ METHODS}

The purpose of this chapter is to describe the methodology used in this study. The study design will be discussed first including sample, setting, and inclusion/exclusion criteria. A discussion of human subjects considerations will follow along with a discussion of measurement including specific instrumentation to assess the study variables. Data collection and data management procedures will be described. Finally, statistical analyses will be delineated including sample size calculation.

\section{Study Design}

This study employed a descriptive, cross-sectional design. Questionnaires were administered via pen and paper and an in-person interview to assess symptoms and timing of help-seeking behavior in individuals diagnosed with lung cancer. Primary study outcomes evaluated the timing of help-seeking behavior, healthcare system distrust, lung cancer stigma, and smoking status.

\section{Sample and Setting}

The target population for this study was lung cancer patients. Participation was dependent upon a participant's eligibility, willingness to participate, and participation in the informed consent process. Interested individuals were 
required to meet the following inclusion criteria: (a) age 22 or older, (b) able to speak and understand the English language, (c) diagnosed with lung cancer as the primary site of cancer and, (d) knowledge of their stage of lung cancer. Although the American Cancer Society (2013) reports that greater than $60 \%$ of lung cancer diagnoses are in individuals age 65 or older, capturing data from adult patients less than 65 years of age provided a more inclusive opportunity to understand predictor variables of delayed help-seeking behavior in lung cancer patients. Exclusion criteria included inability to speak and understand English and lung as a secondary site (not primary) of cancer.

The sample population obtained for analysis represented a convenience sample of lung cancer patients at the multidisciplinary lung cancer clinic at the James Graham Brown Cancer Center (JGBCC) and the Radiation Oncology Clinic at Baptist Hospital East (BHE) Cancer Care Center. The James Graham Brown Cancer Center is located in downtown Louisville, Kentucky within an academic medical center. This is a multidisciplinary lung cancer clinic with one board certified medical oncologist, multiple hematology/oncology resident physicians and fellows, and one nurse practitioner. The Radiation Oncology Clinic at Baptist Hospital East is a community-based, private hospital radiation oncology center with four board certified radiation oncologists.

\section{Human Subjects Considerations}

Letters of support were obtained from the James Graham Brown Cancer Center (JGBCC), and Baptist Hospital East (BHE) and included with an expedited approval request through the University of Louisville's Human Subjects 
Protection Program Office (HSPPO). The University of Louisville HSPPO approved the protocol and study preamble. The protocol and study preamble were then reviewed and approved by the University of Louisville Research Integrity Office (RIO), JGBCC Clinical Scientific Review Committee (CSRC), University of Louisville Hospital Nursing and Interdisciplinary Research Committee, and BHE Nursing Research Council. Approval letters can be found in Appendix A. Participant recruitment materials (recruitment flyer) were approved by the HSPPO. Please see Appendix B for a copy of the IRB-approved recruitment flyer.

The informed consent process was conducted by the investigator in a private exam room, and included a discussion and explanation of the study purpose and goal, study procedures, type of data collected, risks and benefits associated with the study, study personnel contact information, and relevant patient confidentiality and privacy issues according to Polit and Beck (2004). It was expected that study-related procedures would involve minimal risk to participants. Emphasis was placed on the voluntary nature of participation, and that the participant could withdraw at any time without fear of repercussions or negative outcomes secondary to their withdrawal. The participants were informed that compensation would not be provided for participation. The participants were also informed that no identifying information such as names, patient medical record numbers, or social security numbers would be collected. Participants were allowed adequate time to read the study preamble (Appendix C) and ask questions. The investigator answered all questions to the participant's 
satisfaction. Data collection did not proceed until the informed consent process was fully completed. A copy of the study preamble was provided to participants.

There were no conflicts of interest to be disclosed. Specifically, there was no compensation provided by the investigator to the referring JGBCC or BHE physician, nurse, or clinic staff. The investigator was not a clinical practitioner or an employee of JGBCC or BHE. The physicians, nurses, clinic staff, and investigator followed professional and ethical conduct and did not use coercive tactics for participant recruitment. In addition, the JGBCC and BHE clinic staff were not involved in analysis of the research.

\section{Minority and Children Participation}

This study explored the timing of help-seeking behavior in lung cancer patients. Two sites were used to increase the potential for ethnic and socioeconomic diversity in the sample. Approximately $50 \%$ of lung cancer patients are female (ACS, 2013) and constituted the potential for a significant female presence in the study. The literature notes that minority study participants are historically underrepresented in healthcare research (Corbie-Smith et al., 2002; Corbie-Smith et al., 1999; Gamble, 1997; Moseley, Freed, Bullard, \& Goold, 2007). Both recruitment sites had an adequate number of female lung cancer patients as well as minority lung cancer patients for recruitment. The inclusion of multiple ethnicities in the design of this study allowed inclusion of the historically underrepresented minority population. Children were not included in this study because lung cancer is not generally diagnosed in this demographic population. 


\section{Measurement}

To test the specific aims and the research hypothesis, four instruments were administered: the Revised Health Care System Distrust Scale (Appendix E), Cataldo Lung Cancer Stigma Scale (Appendix F), Modified Marlowe-Crowne Social Desirability Scale (Appendix G) and an investigator-developed Timing of Help-Seeking Behavior \& Demographic Questionnaire (THSBDQ) (Appendix H). The dependent (outcome) variable was assessed with an in-person interview. This study focused on healthcare system distrust (continuous data from scores on the Revised Healthcare System Distrust Scale), lung cancer stigma (continuous data from scores on the Cataldo Lung Cancer Stigma Scale), and smoking status (categorical data from the THSBDQ). The dependent variable was time to seek help in lung cancer (time in days). Covariates included social desirability (continuous data from scores on the Modified Marlowe-Crowne Social Desirability Scale), socioeconomic status (categorical data from the THSBDQ), and ethnicity (categorical data from the THSBDQ). A complete listing of variables with their respective measures and associated conceptual model constructs are summarized in Table 3.1 below. 
Table 3.1

Measures of Identified Variables

\begin{tabular}{|c|c|c|}
\hline Variable & Measure & $\begin{array}{c}\text { Conceptual } \\
\text { Model Construct }\end{array}$ \\
\hline $\begin{array}{c}\text { Timing of Help-Seeking } \\
\text { Behavior }\end{array}$ & $\begin{array}{c}\text { Timing of } \\
\text { Help-Seeking } \\
\text { Behavior \& } \\
\text { Demographic } \\
\text { Questionnaire } \\
\text { (THSBDQ) }\end{array}$ & $\begin{array}{l}\text { Help-Seeking } \\
\text { Behavior }\end{array}$ \\
\hline Healthcare System Distrust & $\begin{array}{c}\text { Revised } \\
\text { Health Care } \\
\text { System } \\
\text { Distrust Scale } \\
\text { (Shea et al., } \\
\text { 2008) }\end{array}$ & $\begin{array}{c}\text { Sociodemographic } \\
\text { Variable }\end{array}$ \\
\hline Lung Cancer Stigma & $\begin{array}{l}\text { Cataldo Lung } \\
\text { Cancer } \\
\text { Stigma Scale } \\
\text { (Cataldo et al., } \\
\text { 2011) }\end{array}$ & $\begin{array}{c}\text { Sociodemographic } \\
\text { Variable }\end{array}$ \\
\hline Smoking Status & THSBDQ & $\begin{array}{l}\text { Sociodemographic } \\
\text { Variable }\end{array}$ \\
\hline Social Desirability & $\begin{array}{c}\text { Modified } \\
\text { Marlowe- } \\
\text { Crowne Social } \\
\text { Desirability } \\
\text { Scale } \\
\text { (Strahan \& } \\
\text { Gerbasi, } \\
\text { 1972) } \\
\end{array}$ & N/A (covariate) \\
\hline Socioeconomic Status & THSBDQ & $\begin{array}{c}\text { Sociodemographic } \\
\text { Variable }\end{array}$ \\
\hline Ethnicity & THSBDQ & $\begin{array}{c}\text { Sociodemographic } \\
\text { Variable }\end{array}$ \\
\hline
\end{tabular}

\section{Dependent Variable}

Timing of help-seeking behavior.

Timing of help-seeking behavior was assessed with an in-person interview using the investigator-developed Timing of Help-Seeking Behavior \& 
Demographic Questionnaire (THSBDQ). The THSBDQ was comprised of two sections: (1) questions regarding the timing of help-seeking behavior and, (2) demographic questions. The first section of the THSBDQ was a seven-question component that asked the participant to recall the timing of the first symptom experienced, type of symptom(s) experienced, timing of the first time they sought help, timing of the first time they received help, timing of when they were diagnosed with lung cancer, initial stage at diagnosis, and hindsight symptoms after diagnosis. If the participant had difficulty recalling the events, key event mapping was used. Key event mapping uses a calendar to assist the participant in recall by asking the participant to remember in relation to key personal events and common key events on a calendar (Molassiotis et al., 2010). The recollection of the first symptom, the first time seeking help, and the time that help was received was recorded in month/day/year format. They were also asked if, after they were diagnosed, they realized there were symptoms in hindsight prior to their diagnosis. If the participant answered affirmatively, those symptoms were recorded.

Time to seek help in lung cancer was the dependent variable and was measured using the date difference between the date of seeking help for lung cancer symptoms and the date of first symptom(s) experienced. The date reported as the first symptom experienced was recorded in a month/day/year format. The date reported as the first time they sought help for the symptom(s) was recorded in a month/day/year format. In the case of individuals that could only recall a month and year, the first day of that month was used for data entry 
purposes. In lieu of a specific date for the remaining questions for that participant, the investigator asked the participant to report the time difference in weeks or months. For data entry purposes, the first day of the month reported by the participant was used and the investigator calculated the number of weeks or months using the reported timeframe.

\section{Independent Variables}

The independent variables were healthcare system distrust, lung cancer stigma, and smoking status.

Distrust. Healthcare system distrust was measured by the Revised Health Care System Distrust Scale (Shea et al., 2008). This scale was the evolution of its predecessor, the Health Care System Distrust Scale (Rose et al., 2004). The Revised Health Care System Distrust Scale measures health care system distrust to provide insight into the healthcare system as a whole, or "institutional" trust. The Revised Health Care System Distrust Scale attempts to examine the multidimensional nature of healthcare system distrust consistent with the proposed multidimensional nature of trust and distrust (Shea et al., 2008). The Health Care System Distrust Scale will be discussed briefly as a foundation for understanding the development of the Revised Health Care System Distrust Scale, which will follow.

Health care system distrust scale. The Health Care System Distrust Scale was originally developed in 2004 as an 11-item five-point Likert-type instrument that was "to measure health care system distrust and to examine. . .whether health care system distrust functioned as a one-dimensional or 
multidimensional construct" (Rose et al., 2004, p. 58). The Health Care System Distrust Scale was developed in response to the proposition that distrust may serve as a significant barrier to an individual seeking medical care, participating in medical research, or maintaining follow-up care to ensure effective treatment of a disease process.

The Health Care System Distrust Scale underwent two phases of development: development of a conceptual model of healthcare-related trust and distrust and development of the instrument. Four domains of distrust were identified: fidelity, competence, confidentiality, and honesty/informed consent. The authors hypothesized an inverse relationship between trust in personal physician and distrust of the healthcare system as well as a positive correlation between ethnic minorities and distrust of the healthcare system based upon historical evidence and evidence suggested by the literature (Rose et al., 2004). The final scale resulted in 10 items representing the four conceptual domains yielding a Cronbach's coefficient alpha internal consistency reliability of .75. Principal components analysis tested construct validity and yielded one component with an eigenvalue of 3.17 explaining $32 \%$ of the total variance. The rotated loadings for the 10 scale items ranged from .38 to .73 .

Revised health care system distrust scale. Shea et al. (2008) revisited the Health Care System Distrust Scale as evidence mounted suggesting a multidimensional nature of distrust. The domains of technical competence and value congruence were postulated and the investigators noted, "prior measures of health care system distrust have not reflected this multidimensional structure 
and may be limiting research into the role of health care system distrust in health and health care in the US" (Shea et al., 2008, p. 727). Therefore, a three-phase study was undertaken including focus groups, pilot testing and cross-sectional telephone survey to develop a revised scale of healthcare system distrust.

Phase One involved 12 focus groups with a total of 115 ethnically diverse individuals guiding the participants through four major topics: (a) defining the healthcare system, (b) consideration of trust versus distrust as opposite ends of a continuum, (c) elements/actions associated with distrust and, (d) elements/actions associated with trust. The original conceptual model was refined based upon these results (Shea et al., 2008).

Phase Two involved the task of item reduction. Seventy-five items were piloted with 34 participants in the waiting rooms of primary care provider practices for domain and dimensional representation and ambiguity. Phase Two resulted in a scale item reduction from 75 to 26 items.

Phase Three involved psychometric testing, analysis, and further item reduction through telephone survey "to explore the psychometric characteristics of the final scale and to test hypotheses about the relationship between distrust and race" (Shea et al., 2008, p. 728). A random sample of 845 individuals was chosen and 264 participated (although nine did not provide ethnicity information so were excluded from the final analysis). The final sample results included $56 \%$ African-American, 36\% Caucasian, and 8\% from other ethnic groups. The original scale in Phase Three resulted in eight items with a Cronbach's coefficient alpha of .83 with item-total correlations ranging from .5 to .7 . Principal 
components analysis supported the multidimensional nature of healthcare system distrust identifying two subscales consistent with the proposed domains of competence (Cronbach's coefficient alpha $=.77$ ) and values (Cronbach's coefficient alpha $=.73$ ) with four items loading on each domain. A ninth item was added to the final scale to address the subtheme of equity by beginning with a stem of "Patients..." versus "The healthcare system..." yielding a final Cronbach's coefficient alpha of .85 for the nine-item scale and .75 for the fiveitem values subscale. The nine-item, five-point Likert scale has a range from nine (reflecting low levels of healthcare system distrust) to 45 (reflecting high levels of healthcare system distrust).

Shoff and Yang (2012) note that the researchers of the Revised Health Care System Distrust report addressing a multidimensional structure in their instrument. However, Shoff and Yang purport the instrument is actually a dual dimensional scale focused on technical competence and values congruence. While it is a more improved scale with adequate reliability and validity, there are shortcomings such as not accounting for potential important correlates such as insurance status and access to healthcare. In addition, the developers of the scale did not make an explicit distinction between "distrust" and "dissatisfaction". Finally, the dual dimensionality of the total scale might not capture the whole concept of healthcare system distrust such as research-related distrust, health policy issues, patient privacy issues, and perceptions of transparency (Shoff \& Yang, 2012). 


\section{Lung cancer-related stigma.}

Cataldo lung cancer stigma scale. The CLCSS was used to measure lung cancer stigma and Cataldo et al. (2011) defines:

health-related stigma [as] a perceived stigma that has been defined as a personal experience characterized by exclusion, rejection, blame, or devaluation that results from anticipation of an adverse judgment [and notes that] stigma in lung cancer is based on the belief that the patient's behavior was the cause of the cancer. ( $p$. E46)

The Cataldo Lung Cancer Stigma Scale (CLCSS) was developed to measure perceived stigma in lung cancer patients. This scale was derived from the HIV Stigma Scale (Berger, Ferrans, \& Lashley, 2001) because of similarities in experience of perceived self-infliction of the disease process (Cataldo et al., 2011).

Cataldo et al. (2011) used the conceptual model of perceived stigma from the HIV Stigma Scale as a guide noting "the perceived stigma of lung cancer occurs in the context of two factors: a person's perception of societal attitudes toward both smoking and lung cancer and a personal knowledge of having lung cancer" (p. E47). The CLCSS was modified for use in a population of lung cancer patients. Content validity was ensured by the inclusion of seven experts on the concept of stigma, representing the disciplines of sociology, psychology, oncology, and nursing as reviewers. If an item was rejected by more than one of the seven reviewers, it was discarded or rewritten. This resulted in the reduction of the original 45 -item scale to 37 items. Nine additional items were developed, reviewed and accepted by the content experts resulting in the final 46 -item scale. 
The CLCSS is based upon a 4-point Likert-type scale anchored by 1 (strongly disagree) and 4 (strongly agree).

Psychometric testing of the final scale was undertaken with 186 individuals with lung cancer (Cataldo et al., 2011). Construct validity was supported and involved exploratory factor analysis which revealed four underlying subscales: (a) stigma and shame, (b) social isolation, (c) discrimination and, (d) smoking. The four-factor solution explained $57 \%$ of the variance. According to Stevens (2009), greater than $50 \%$ cumulative explained variance is considered 'excellent' in factor analysis. Using an eigenvalue greater than one criterion for the subscales and a loading cutoff of .35, 43 of the 46 items were retained because they loaded on one of the four subscales.

Criterion-related validity was supported by examining the relationship of the 43-item instrument with the pre-identified related constructs (Cataldo et al., 2011): self-esteem (measured using the Rosenberg Self-Esteem Scale), depression (measured using the Center for Epidemiologic Studies-Depression Scale), social support and social conflict (both measured using the Social Support indices), and quality of life (measured using the Quality of Life Inventory). Internal consistency reliability (Cronbach's coefficient alpha) of the total 43-item CLCSS was .98. The Cronbach's coefficient alphas for the four subscales were: .95 (discrimination), .75 (smoking), .98 (social isolation), and .97 (stigma and shame). The CLCSS was shortened to a 31-item stigma scale to decrease patient burden after it was determined the scale could be shortened while maintaining an adequate internal consistency reliability (Cataldo et al., 
2011). Internal consistency reliability of the shortened 31-item CLCSS yielded a Cronbach's coefficient alpha of .96 with Cronbach's coefficient alphas of the four subscales of .92 (discrimination), .75 (smoking), .96 (social isolation), and .97 (stigma and shame). The 31-item, four-point Likert scale has a range from 31 (reflecting low levels of lung cancer stigma) to 124 (reflecting high levels of lung cancer stigma).

The CLCSS is a relatively new instrument and, to date has been used in one published study by Lee and Kim (2011) in which the relationships between stigma, distress, and quality of life were examined in lung cancer patients. The study revealed a positive correlation between lung cancer stigma and anxiety ( $r=$ $.37, p<.001)$ and depression $(r=.44, p<.001)$ and a negative correlation between lung cancer stigma and quality of life $(r=-.26, p=.003)$ and function $(r$ $=-.40, p<.001)$. The study concluded that individuals with lung cancer experience stigma and distress as a negative influence on their quality of life (Lee \& Kim, 2011), and further supported the reliability of the instrument.

Smoking status. Smoking status was assessed and classified as a never smoker, former smoker, or current smoker at the time of diagnosis. It was measured using the THSBDQ. The following three demographic questions helped establish smoking status at diagnosis: (1) Have you smoked at least 100 cigarettes in your entire life?; (2) At the time you were diagnosed with lung cancer, were you a smoker?; (3) Do you now smoke cigarettes every day, some days, or not at all?; and, (4) How long has it been since you last smoked cigarettes regularly? 


\section{Covariates}

Social desirability. Social desirability was measured using the Modified Marlowe-Crowne Social Desirability Scale (Strahan \& Gerbasi, 1972). This scale is a shortened version of the original 33-item scale (Crowne \& Marlowe, 1960). The modified scale is comprised of 20 scale items. Psychometric testing of the modified scale yielded Kuder-Richardson 20 (K-R 20) reliability coefficients of .78 in one sample $(\mathrm{N}=64)$ of males and .73 in one sample $(\mathrm{N}=130)$ of females. The original 33-item Marlowe-Crowne Social Desirability Scale was tested in the same samples and yielded K-R 20 reliability coefficients of .83 and .73 respectively.

Socioeconomic status and other demographic data. The Timing of Help-Seeking Behavior \& Demographic Questionnaire (THSBDQ) was used to collect socioeconomic and demographic variable data. Socioeconomic data were collected including income level (less than $\$ 25,000, \$ 25,000-\$ 50,000$, greater than $\$ 50,000$ ) and a dichotomous question assessing the participant's perception of financial status: "Do you feel your income is adequate to meet your basic needs?". Additional demographic variables such as age (in years), gender, marital status, health insurance status, and ethnicity were collected.

\section{Data Collection Procedures}

Data collection began after approval of the study protocol was obtained from all approving bodies including the Institutional Review Board (IRB) at the University of Louisville and both participating hospitals. Participants were recruited on a rolling basis from JGBCC over a nine-week period from December 
2012 to February 2013 and from BHE over a four-week period, from January to February 2013.

Lung cancer patients being seen for a follow-up or treatment appointment at JGBCC and BHE were informed of the opportunity to be involved in a research study either by the oncologist, nurse practitioner, or clinic nurse. All clinic nurses, nurse practitioner, and oncologist were provided information about the research study and asked to identify potential eligible participants. Potential study participants were contacted during a follow-up or treatment appointment in the clinic by the clinic staff. If the potential study participant indicated interest, the investigator was invited into the room to introduce the study and invite them to participate. Interest in the study was further assessed. Inclusion and exclusion criteria were assessed and if they met the inclusion criteria, they were invited to participate in the study. If they indicated they wanted to participate in the study, the investigator reviewed detailed information about the study. If the potential study participant indicated they were still interested, a copy of the study preamble was provided for their review and the investigator addressed any further questions. The potential study participant was then asked if they wanted to participate in the study. If the individual expressed that they did wish to participate, data collection began. If the individual expressed that they did not wish to participate, they were thanked for their time. If the individual expressed that they did wish to participate but were unable to stay for data collection, an appointment was scheduled with them to meet back in the clinic to perform data collection. All data collection forms can be found in Appendices D through G. 
Data collection was performed in an exam room or private area within the clinic to offer privacy. There was not an option to take the surveys home. There were instances in which an interested participant did not have time to complete the data collection process at the initial meeting. In those cases, the participant was given a copy of the study preamble and recruitment flyer to take home and a mutually agreed upon time was scheduled when the participant would return to the clinic. The investigator met the participant at their follow-up appointment time in the clinic to complete the study data collection procedure.

The investigator conducted all data collection sessions in one sitting lasting from 10 to 30 minutes. Study participants completed three surveys and an interview. A standardized protocol was followed for each data collection session including detailed explanation of the study and informed consent process using a study preamble, time allotment for questions, and provision of study preamble copy.

The investigator began with the first part of the interview using the first section of the investigator-developed Timing of Help-Seeking Behavior \& Demographic Questionnaire (THSBDQ). The investigator collected information about timing of help-seeking behavior with first lung cancer symptom(s). Specifically, the participant was asked about the timing of their first symptom, the type of symptom(s) experienced, timing of the first time they sought help, timing of the first time they received help, initial stage at diagnosis, and hindsight symptoms the participant may have experienced after being diagnosed with lung cancer. After the timing of help-seeking behavior interview was conducted, the 
study participant was asked to complete the survey packet. The survey packet included the (a) Revised Health Care System Distrust Scale, (b) Modified Marlowe-Crowne Social Desirability Scale and, (c) Cataldo Lung Cancer Stigma Scale. For consistency, the survey packet was packaged in identical order. In addition, the survey instruments were numerically coded to de-identify the instruments from their respondents. No names were recorded on any paperwork kept by the investigator. Depending upon participant preference, the participant either completed the surveys by hand with a pen or the participant was read the survey by the investigator while the investigator recorded the answers. After completion, the investigator reviewed the three surveys for completeness and the remainder of the interview (second section of the THSBDQ) was conducted.

After the completion of the survey packet, the investigator reviewed the surveys for any potential missing data. If missing data were noted, clarification to determine if the missing data were an oversight versus intentional was assessed. Emphasis on the voluntary nature of the participant's answers was acknowledged to the participant from the investigator. Finally, participants were thanked for their participation at the conclusion of the data collection session.

\section{Data Management Procedures}

Unique numeric identifiers were assigned to each participant's survey packet to maintain anonymity. Each participant's survey packet had a three-digit number. All study related materials (i.e. completed survey packets) were stored in the investigator's secured, limited access office. Furthermore, all research data 
were collected, maintained, and entered for statistical analysis by the investigator.

\section{Accuracy of Input}

At the end of each data collection day, the investigator manually entered the data into the Statistical Program for Social Sciences (SPSS) software version 20.0 (SPSS, 2012) to create an original data file. After the conclusion of recruitment and closure of the study, the investigator manually entered the data again in a separate file. Both the original data file and the second data entry file were then merged via SPSS and inspected for deviations. Any deviations noted were then recorded and compared to the original survey completed by the study participant. Corrections to data entry were completed and a master data file was saved and used for statistical analyses.

\section{Statistical Analyses}

\section{Descriptive Analysis}

All data analyses were performed using SPSS software version 20.0. Demographics were obtained for the entire sample, and descriptive statistics were calculated including frequencies, means, standard errors, and ranges on all variables. Handling of missing data will be discussed in a separate section below.

\section{Reliability of Scales}

Cronbach's alphas were calculated on all scales and subscales and evaluated. 


\section{Inferential Analysis}

Pearson product moment correlation coefficients were used to examine the relationship among the independent variables (healthcare system distrust and lung cancer stigma) and the dependent variable (timing of help-seeking behavior) (Aim 1). Kruskal-Wallis $\mathrm{H}$ test was used to compare the three smoking status groups (never smokers, former smokers, and current smokers) to the continuous dependent variable, time to seek help in days. In addition, Chi-square tests for independence were used to examine the relationship between smoking status and the dependent variable (timing of help-seeking behavior) after categorically grouping the dependent variable (Aim 1). The bivariate relationships of socioeconomic status, social desirability, and ethnicity were examined for the independent variables and dependent variable through one-way analysis of variance and Pearson product moment correlation coefficients (Aim 2). Finally, multivariable analysis was used to determine the predictive power of the model for timing of help-seeking behavior in lung cancer patients through hierarchical multiple regression (Aim 3).

\section{Sample Size Calculation}

To determine an appropriate sample size, a significance level of .05 and a medium effect size was selected. An apriori power analysis for multiple regression to detect a significant difference was approximately $80 \%$ if the critical F value was 2.32 or greater required a target sample $(N)$ of 92 . Cohen (1992) considers ratios of this magnitude to constitute a medium effect size. Power estimates were obtained using G*Power version 3.1.4 (Faul, Erdfelder, Lang, \& 
Buchner, 2009). In addition, with three independent variables (the maximum number of variables used in the regression analysis), Tabachnick and Fidell's (2001) "rule of thumb" formula $(N \geq 50+8 m$, where $m$ is the number of independent variables in the statistical calculation) suggested that 74 study participants were needed to test correlations and regression analysis, assuming an alpha of .05 , power of .80 , and a medium effect size.

The enrollment period for this study was nine weeks. The first study participant was consented December 14, 2012, and the final study participant was consented and completed the data collection process on February 15, 2013.

\section{Missing Data}

Knapp (1998) defines missing data as occurring

when there is no information for one or more subjects on one or more variables in a research study [with] the principle consequence of missing data is that the $N$ for each variable is not the same as the sample size $N$ (p. 240).

Knapp outlines four reasons for missing data to occur: (a) unintentional lack of cooperation, (b) refusal to provide certain information, (c) malicious intent and, (d) omissions secondary to clerical error. Knapp (1998) has identified five coping strategies for missing data: (a) prevention, (b) deletion of additional data, (c) imputation of estimates, (d) working around the missing data and, (e) studying it.

Knapp (1998) recommends the best way to handle missing data is through prevention. This study incorporated a process of prevention by examining the completed surveys prior to the study participant's departure to examine the documents for missing data. In this study, there were no omissions in survey answers. 


\section{Data Preparation}

\section{Normality}

Both correlations and multivariable regression analysis assume multivariable normal distributions for continuous variables. Multivariable normality refers to all variables and all linear combinations of variables are normally distributed. Determining multivariable normality begins with assessing for violations of univariate normality. This refers to assessment of the distribution of individual variables, and can be violated when the skew and kurtosis of the variable deviates from zero (Tabachnick \& Fidell, 2001).

Distributions of the continuous variables (time to seek help in days, healthcare system distrust, lung cancer stigma, and social desirability) were assessed for normality using skewness and kurtosis statistics, histograms, and normal probability plots. Tabachnick and Fidell (2001) recommend using alpha levels of .01 or .001 (critical values \pm 2.58 and \pm 3.29 , respectively) to evaluate the significance of skewness and kurtosis with small and moderate sample sizes. Table 3.2 provides a summary of skewness and kurtosis statistics for the continuous variables that were examined in this study. The raw data for the dependent variable, time to seek help in days, revealed a skewness of 2.07 and review of the histogram revealed a positively skewed distribution. The decision was made to apply a Lg10 transformation to time to seek help in days, resulting in a more normally distributed dependent variable. The skewness of the Lg10 transformed data was -.816 . 
Table 3.2

Normality Statistics for Continuous Variables

\begin{tabular}{lccc}
\hline Variable & $\boldsymbol{M} \pm \mathbf{S D}$ & Skewness & Kurtosis \\
& & & \\
Total RHCSDS Score & $30.37 \pm 6.11$ & .044 & -.129 \\
Total CLCSS Score & $68.60 \pm 11.49$ & -.241 & 2.139 \\
$\begin{array}{l}\text { Time to Seek Help in } \\
\text { Days (Raw Data) }\end{array}$ & $69.69 \pm 81.75$ & 2.072 & 4.526 \\
$\begin{array}{l}\text { Time to Seek Help in } \\
\text { Days (Lg 10 }\end{array}$ & $1.51 \pm .662$ & -.816 & .034 \\
Transformed Data) & & & \\
\end{tabular}

Note. RHCSDS = Revised Healthcare System Distrust Scale; CLCSS = Cataldo Lung Cancer Stigma Scale

Histograms and normality plots were also examined for all continuous variables. See Figure 3.1 for graphic representation of normality of independent variables, Revised Healthcare System Distrust Scale and Cataldo Lung Cancer Stigma Scale. These graphs, which are suggested to be more meaningful indicators of deviation from normality than skewness and kurtosis (Tabachnick \& Fidell, 2001), supported the conclusion that all continuous variables except time to seek help in days were normally distributed. Log transformation of time to seek help in days corrected the lack of normality (see Figure 3.2). 

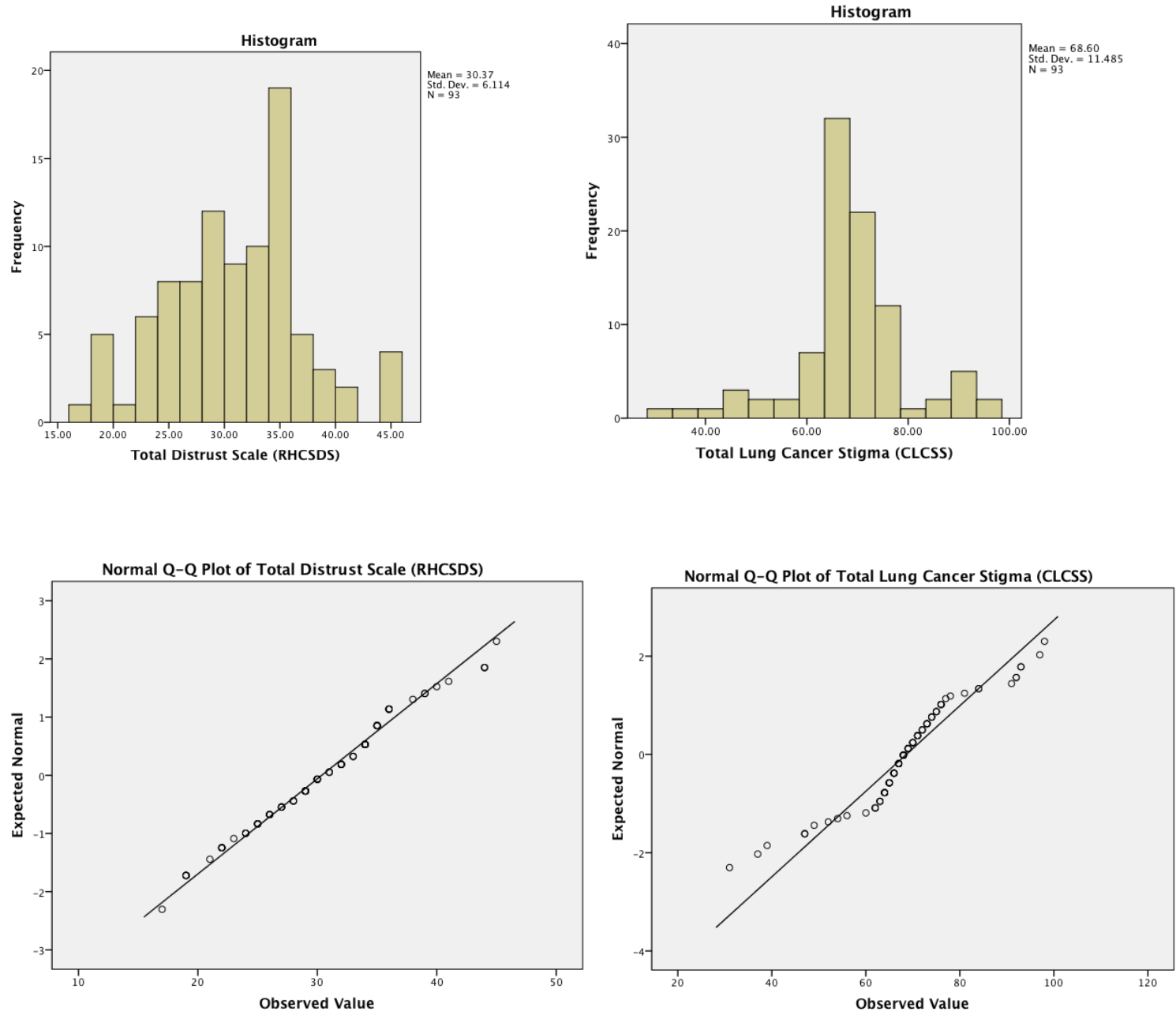

Figure 3.1. Graphic representation of normality of independent variables (RHCSDS total scores and CLCSS total scores) 


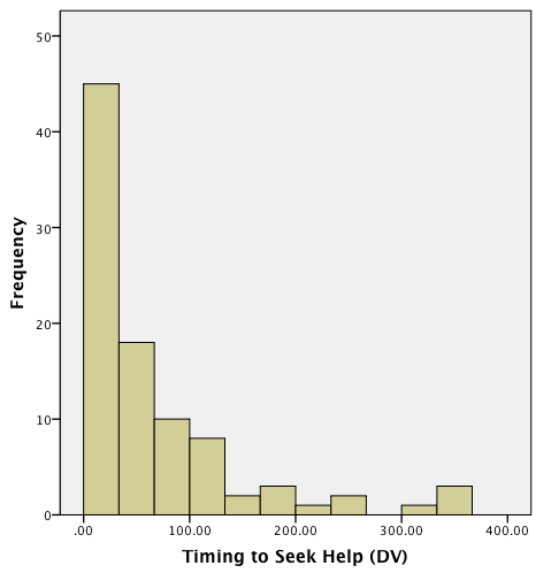

Raw data $(N=93)$

Time to Seek Help

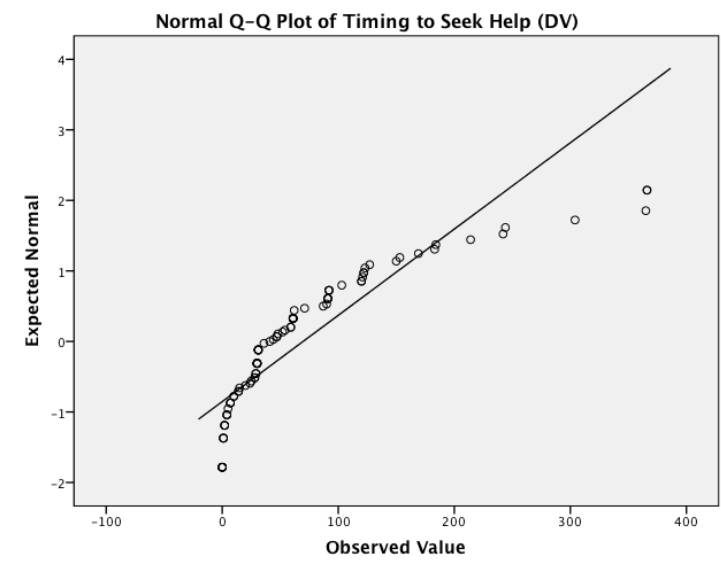

Raw data $(N=93)$

Time to Seek Help

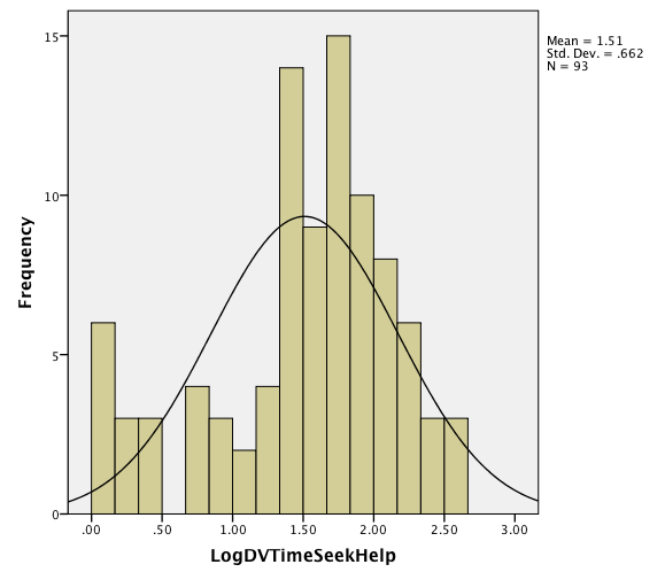

Transformed data $(N=93)$

Time to Seek Help

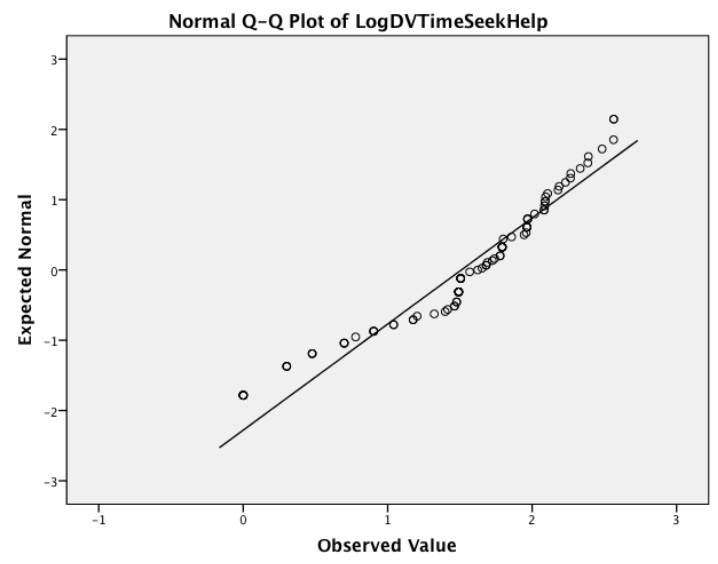

Transformed data $(N=93)$

Time to Seek Help

Figure 3.2. Histograms and Q-Q plots of Time to Seek Help: Raw versus transformed data 


\section{Multicollinearity}

Multicollinearity occurs when there are moderate to high intercorrelations among independent variables (Stevens, 2009). Collinear variables provide redundant information making it difficult to separate the effects of the independent variables. Statistically, multicollinearity inflates the size of error terms and restricts the size of the multivariable correlation (Stevens, 2009; Tabachnick \& Fidell, 2001).

Three indices were used to screen for multicollinearity for the continuous variables: tolerance, variance inflation factor (VIF), and condition index. Stevens (2009) notes that tolerance values close to one indicate the variable is not linearly related to the other independent variables, while values close to zero indicate a strong relationship between that variable and the other independent variables. The VIF is the reciprocal of tolerance. The VIF measures increases in variance of the coefficients due to the correlations among the independent variables (Stevens, 2009). Finally, the condition index measures the "tightness or dependency" of a variable on the other variables (Tabachnick \& Fidell, 2001). The condition index represents the square root of the ratio of the largest eigenvalue to each successive eigenvalue, and values greater than 15 suggest a potential problem with multicollinearity. All indices (VIF, tolerance, and the condition index) suggested that multicollinearity was not an issue among the independent variables in this study. Refer to Table 3.3 for screening of multicollinearity for continuous independent variables. 
Table 3.3

Screening for Multicollinearity for Continuous Independent Variables

\begin{tabular}{lccc}
\hline Variable & Tolerance & VIF & $\begin{array}{c}\text { Condition } \\
\text { Index }\end{array}$ \\
Total RHCSDS Score & .672 & 1.489 & 7.254 \\
Total CLCSS Score & .669 & 1.494 & 9.285
\end{tabular}

Note. RHCSDS = Revised Healthcare System Distrust Scale; CLCSS = Cataldo Lung Cancer Stigma Scale

\section{Summary}

Through a cross-sectional design, this study examined the influence of healthcare system distrust, lung cancer stigma, and smoking status on the timing of help-seeking behavior in individuals with symptoms suggestive of lung cancer. Understanding the factors that influence the timing of help-seeking behavior can address one aspect of a multifactorial problem and be foundational to future intervention work to decrease the time to diagnosis. Lung cancer symptoms are often present, but individuals may not recognize them as indicative of lung cancer leading to delayed help-seeking behavior and advanced stage when diagnosed. Because lung cancer is the deadliest cancer worldwide, decreasing the time to diagnosis can decrease patient morbidity from lung cancer. 


\section{CHAPTER FOUR}

\section{RESULTS}

The purpose of this study was to explore the influence of healthcare system distrust, lung cancer stigma, and smoking status on the timing of helpseeking behavior in individuals with symptoms suggestive of lung cancer. This study examined three specific aims and 10 related hypotheses. This chapter begins with a description of sample characteristics followed by results of the statistical analyses presented in order of the specific aims and research hypotheses.

A total of 94 participants were enrolled into the study after completing the informed consent process. A total of 94 participants completed the data collection procedure. Preliminary statistical analysis was performed and one case was excluded from the final statistical analysis because it represented an extreme outlier on the dependent variable of time to seek help in days for symptoms suggestive of lung cancer. Data obtained on the survey instruments were entered into SPSS Version 20.0. No missing data were noted in the data set. To reduce skewness and kurtosis of the dependent variable, a log transformation (Lg10) was performed. 


\section{Sample Characteristics}

Study participants were recruited from two hospitals in Louisville, Kentucky $(n=93)$. The majority of the participants $(n=72 ; 77.4 \%)$ were from an academic hospital setting (James Graham Brown Cancer Center) and the remaining sample $(n=21 ; 22.6 \%)$ was recruited from a community hospital setting (Baptist Hospital East). The mean age at lung cancer diagnosis for the study sample was 62.0 years. $37.6 \%$ were male and $62.4 \%$ were female. The study sample was $81.7 \%$ Caucasian, $17.2 \%$ African-American, and $1.1 \%$ Hispanic.

Approximately one-third (35.5\%) of the sample reported a college graduate level education (14 years of education or higher), with $21.5 \%$ reporting some college, and $39.8 \%$ reported a high school education level. Only $3.2 \%$ reported having less than 12 years of education. The majority of the sample was married $(64.5 \%)$ at the time of their lung cancer diagnosis, with $4.3 \%$ never married, $17.2 \%$ divorced, and $14.0 \%$ widowed. Employment status revealed the majority of the sample was retired $(52.7 \%)$ when diagnosed with lung cancer. $24.7 \%$ were employed full-time, $7.5 \%$ were employed part-time, $3.2 \%$ were selfemployed, and $11.8 \%$ were unemployed. Insurance status at the time of their lung cancer diagnosis revealed $43.0 \%$ were insured by Medicare, while $43.0 \%$ were insured by private health insurance, Medicaid insured $1.1 \%$, and $12.9 \%$ were uninsured at the time of diagnosis. The majority of individuals sought help for symptoms suggestive of lung cancer in less than 30 days (42\%). However, $29 \%$ of individuals sought help between 31 and 90 days and an equal number of 
individuals sought help greater than 90 days (29\%). Characteristics of the participants are shown in Table 4.1. 
Table 4.1

Descriptive Data of Study Participants - Categorical Variables

\begin{tabular}{|c|c|}
\hline Variable & $\begin{array}{c}\text { Study Participants } \\
\mathrm{N}=93(\%)\end{array}$ \\
\hline \multicolumn{2}{|l|}{ Gender } \\
\hline Male & $35(37.6 \%)$ \\
\hline Female & $58(62.4 \%)$ \\
\hline \multicolumn{2}{|l|}{ Ethnicity } \\
\hline Caucasian & $76(81.7 \%)$ \\
\hline African-American & $16(17.2 \%)$ \\
\hline Hispanic & $1(1.1 \%)$ \\
\hline \multicolumn{2}{|l|}{ Education } \\
\hline Less than High School & $3(3.2 \%)$ \\
\hline High School Graduate & $37(39.8 \%)$ \\
\hline Some College & $20(21.5 \%)$ \\
\hline College Graduate or Higher & $33(35.5 \%)$ \\
\hline \multicolumn{2}{|l|}{ Marital Status } \\
\hline Never Married & $4(4.3 \%)$ \\
\hline Now Married & $60(64.5 \%)$ \\
\hline Divorced & $16(17.2 \%)$ \\
\hline Widowed & $13(14.0 \%)$ \\
\hline \multicolumn{2}{|l|}{ Employment Status } \\
\hline Full-Time & $23(24.7 \%)$ \\
\hline Part-Time & $7(7.5 \%)$ \\
\hline Self-Employed & $3(3.2 \%)$ \\
\hline Unemployed & $11(11.8 \%)$ \\
\hline Retired & $49(52.7 \%)$ \\
\hline \multicolumn{2}{|l|}{ Insurance Status } \\
\hline Medicare & $40(43.0 \%)$ \\
\hline Medicaid & $1(1.1 \%)$ \\
\hline Private Health Insurance & $40(43.0 \%)$ \\
\hline Uninsured & $12(12.9 \%)$ \\
\hline \multicolumn{2}{|l|}{ Time to Seek Help } \\
\hline Less than 30 days & $39(42.0 \%)$ \\
\hline 30 to 90 days & $27(29.0 \%)$ \\
\hline Greater than 90 days & $27(29.0 \%)$ \\
\hline
\end{tabular}


The majority of the participants were advanced stage lung cancer with greater than three-fourths of the sample diagnosed at stage $3(31.2 \%)$ or stage 4 lung cancer (46.2\%). The remaining sample was comprised of $15.1 \%$ stage 2 lung cancer patients and $7.5 \%$ stage 1 lung cancer patients. Smoking status revealed fairly equal distributions with $32.3 \%$ never smokers, $35.5 \%$ past smokers, and $32.3 \%$ current smokers at diagnosis. See Table 4.2 for descriptive data on lung cancer stage and smoking status. 
Table 4.2

Descriptive Characteristics of Study Participants - Lung Cancer Stage at

Diagnosis and Smoking Status

Variable

Stage at Diagnosis $(\mathrm{N}=93)$

Stage 1

Stage 2

Stage 3

Stage 4

Smoker at Diagnosis $(\mathrm{N}=93)$

Yes

No

Smoking Status $(\mathrm{N}=93)$

Never Smoker

Past Smoker

Current Smoker

Do you now smoke? $(\mathrm{N}=63)$

Every Day

Some Days

Not at All

How long since last smoked regularly? $(\mathrm{N}=63)$

Within the past month

Within the past 3 months

Within the past 6 months

Within the past year

Within the past 5 years

Within the past 10 years

10 years or more

Packs per day (PPD) of current smokers at diagnosis $(\mathrm{N}=30)$

Less than 1 PPD

1 PPD

1.5 PPD

2 PPD

3 PPD
Study Participants

$7(7.5 \%)$

$14(15.1 \%)$

$29(31.2 \%)$

$43(46.2 \%)$

$30(32.3 \%)$

$63(67.7 \%)$

$30(32.3 \%)$

$33(35.5 \%)$

$30(32.3 \%)$

$6(6.5 \%)$

$4(4.3 \%)$

$53(57.0 \%)$
$12(12.9 \%)$

$3(3.2 \%)$

$5(5.4 \%)$

$4(4.3 \%)$

$8(8.6)$

$10(10.8 \%)$

$21(22.6 \%)$

$$
\begin{gathered}
5(5.4 \%) \\
12(12.9 \%) \\
1(1.1 \%) \\
10(10.8 \%) \\
2(2.2 \%)
\end{gathered}
$$


While many participants experienced more than one symptom, the most prevalent initial symptom reported by participants was cough or respiratory symptoms (47.3\%). Seventeen percent of participants reported two symptoms and $5.4 \%$ of participants reported three symptoms when first seeking help prior to their lung cancer diagnosis. Other reported initial symptoms in order of prevalence reported in this sample included fatigue (22.6\%), hemoptysis (12.9\%), musculoskeletal pain $(9.7 \%)$, weight loss $(8.6 \%)$, chest pain $(6.5 \%)$, headache $(5.4 \%)$, neurologic changes $(4.3 \%)$, allergy symptoms $(3.2 \%)$, and neck swelling or mass (2.2\%). Three percent of the participants did not experience symptoms, and $2.2 \%$ of the participants were diagnosed secondary to abnormal results noted during routine laboratory studies. See Table 4.3 for descriptive data of the initial symptoms experienced by study participants. 
Table 4.3

Prevalence of Symptoms Experienced by Study Participants

Symptom

Study Participants

Cough/Respiratory Symptoms

$44(47.3 \%)$

Fatigue

$21(22.6 \%)$

Hemoptysis

$12(12.9 \%)$

Musculoskeletal Pain

$9(9.7 \%)$

Weight Loss

$8(8.6 \%)$

Chest Pain

$6(6.5 \%)$

Headache

$5(5.4 \%)$

Neurologic Changes

$4(4.3 \%)$

Allergy Symptoms

$3(3.2 \%)$

Neck Swelling or Mass

$2(2.2 \%)$

Abnormal Laboratory Studies

$2(2.2 \%)$

No Symptoms

$3(3.2 \%)$

Note: Initial symptom reported by participants 


\section{Reliability Statistics}

Analysis was conducted to assess the internal consistency reliability for

the Revised Health Care System Distrust Scale (RHCSDS) and the Cataldo Lung Cancer Stigma Scale (CLCSS). Reliability statistics for all total scales and their subscales are provided in Table 4.4. Cronbach's alpha for the Revised Healthcare System Distrust Scale (.91) and Cataldo Lung Cancer Stigma Scale (.95) were strong as each exceeded the desired minimum of .70 (Stevens, 2009). 
Table 4.4

Descriptive Statistics and Reliability of the Revised Healthcare System Distrust Scale (RHCSDS) and Subscales and the Cataldo Lung Cancer Stigma Scale (CLCSS) and Subscales

\begin{tabular}{|c|c|c|c|c|c|c|}
\hline Scale/Subscales & $\mathbf{N}$ & $\begin{array}{l}\text { \# of } \\
\text { Items }\end{array}$ & Mean(SD) & $\begin{array}{l}\text { Potential } \\
\text { Range }\end{array}$ & $\begin{array}{l}\text { Actual } \\
\text { Range }\end{array}$ & $\begin{array}{c}\text { Cronbach's } \\
\text { alpha }\end{array}$ \\
\hline RHCSDS & 93 & 9 & $30.37(6.11)$ & $9-45$ & $17-45$ & .91 \\
\hline $\begin{array}{l}\text { Competence } \\
\text { Subscale }\end{array}$ & 93 & 4 & $14.65(2.76)$ & $4-20$ & $5-20$ & .89 \\
\hline Values Subscale & 93 & 5 & $15.72(3.77)$ & $5-25$ & $9-25$ & .84 \\
\hline CLCSS & 93 & 31 & $68.60(11.49)$ & $31-124$ & $31-98$ & .95 \\
\hline $\begin{array}{l}\text { Stigma \& Shame } \\
\text { Subscale }\end{array}$ & 93 & 11 & $23.60(4.21)$ & $11-44$ & $11-34$ & .85 \\
\hline $\begin{array}{l}\text { Social Isolation } \\
\text { Subscale }\end{array}$ & 93 & 10 & 20.11(3.95) & $10-40$ & $10-30$ & .96 \\
\hline $\begin{array}{l}\text { Discrimination } \\
\text { Subscale }\end{array}$ & 93 & 5 & $10.42(2.01)$ & $5-20$ & $5-16$ & .86 \\
\hline $\begin{array}{l}\text { Smoking } \\
\text { Subscale }\end{array}$ & 93 & 5 & $14.47(3.08)$ & $5-20$ & $5-20$ & .89 \\
\hline
\end{tabular}




\section{Healthcare System Distrust, Lung Cancer Stigma, Smoking Status, and Time to Seek Help in Symptoms Suggestive of Lung Cancer}

The first specific aim focused on exploring the influence of healthcare system distrust, lung cancer stigma, and smoking status on the timing of helpseeking behavior in individuals with symptoms suggestive of lung cancer. To address this aim, a bivariate analysis of the preliminary relationships of the dependent variable (time to seek help in days [Lg10]) with each independent variable (healthcare system distrust, lung cancer stigma, and smoking status) was performed. Preliminary analyses were performed to ensure no violation of assumptions of normality, linearity, and homoscedasticity.

\section{Healthcare System Distrust}

The overall mean distrust score and standard deviation for this sample (as measured using the Revised Healthcare System Distrust Scale; RHCSDS) was $30.37+6.11$. The RHCSDS has a range from 9 (reflective of low levels of healthcare system distrust) to 45 (reflective of high levels of healthcare system distrust). Healthcare system distrust (as measured using the RHCSDS) was not related to time to seek help in days $(\operatorname{Lg} 10)(r=.07, p=.532)$. To further explore the relationship of healthcare system distrust and lung cancer stigma with the timing of help-seeking behavior in individuals with symptoms suggestive of lung cancer, one-way between-groups analyses of variance were conducted.

Participants were divided into three groups according to time to seek help in days for analysis (Group 1: 30 days or less; Group 2: 31 to 90 days; Group 3: 91 days 
or greater). Mean scores on healthcare system distrust did not differ across the three groups $(F(2,90)=2.473, p=.090)$ suggesting healthcare system distrust did not serve as a factor for increased time to seek help in lung cancer in this sample. In addition, neither the mean competence subscale score $(F(2,90)=$ $2.917, p=.059)$ nor the mean values subscale score $(F(2,90)=1.734, p=.182)$ differed across time to seek help groups.

To determine if the relationship between distrust and time to seek help differenced across ethnic groups, Pearson product moment correlations were obtained for the total RHCSDS and both subscales with time to seek help by ethnicity. There were no significant relationships between time to seek help in days (Lg10) and the total RHCSDS $(r=.07, p=.534)$, or the competence subscale $(r=.05, p=.662)$ and values subscale $(r=.08, p=.481)$ for Caucasian study participants. Similarly, there was no significant relationship between time to seek help in days $(\operatorname{Lg} 10)$ and the total RHCSDS $(r=.17, p=.542)$, or the two subscales, competence $(r=-.03, p=.914)$ and values $(r=.26, p=.326)$ for African-American participants.

\section{Lung Cancer Stigma}

The overall mean lung cancer stigma score and standard deviation for this sample (as measured using the Cataldo Lung Cancer Stigma Scale; CLCSS) was 68.60+11.49. Lung cancer stigma and time to seek help in days (Lg10) were positively correlated $(r=.27, p=.010)$ such that individuals with higher total stigma scores reported a greater number of days in time to seek help in symptoms suggestive of lung cancer. The CLCSS is comprised of four 
subscales: stigma and shame; social isolation; discrimination; and smoking. The relationship between each subscale and time to seek help in days (Lg10) was investigated as well using Pearson product moment correlation coefficient. There was a weak, positive correlation between the stigma and shame subscale and time to seek help in days $(\operatorname{Lg} 10)(r=.25, n=92, p<.05)$; a weak, positive correlation between the social isolation subscale and time to seek help in days (Lg10), $r=.23, n=92, p<.05$; and, a weak, positive correlation between the smoking subscale and time to seek help in days (Lg10), $r=.22, n=92, p<.05$. However, there was no significant relationship between the discrimination subscale and time to seek help in days (Lg10). See Table 4.4 for the Pearson product moment correlations between total scale scores, associated subscale scores and the dependent variable (time to seek help in days [Lg10]). To understand the results more completely, Pearson product moment correlations were performed on individual scale items and the dependent variable. Only one scale item from the five-item discrimination subscale was significantly correlated with the dependent variable: I worry that people may judge me when they learn I have lung cancer. The remaining items that correlated with the dependent variable were from the stigma and shame, social isolation, and smoking subscales. See Table 4.5 for the correlations of the Cataldo Lung Cancer Stigma Scale items with time to seek help. 
Table 4.5

Correlations of the Cataldo Lung Cancer Stigma Scale Items with Time to Seek Help

\section{Scale Item}

Stigma and Shame Subscale:

I feel guilty because I have lung cancer.

My lung cancer diagnosis was delayed because I put off going to the doctor.

Social Isolation Subscale:

People seem afraid of me because I have lung cancer.

People avoid touching me if they know I have lung cancer.

I was hurt how people reacted to learning I have lung cancer.

I worry about people discriminating against me.

Discrimination Subscale:

I worry that people may judge me when they learn I have lung cancer.

Smoking Subscale:

Lung cancer is viewed as a self-inflicted disease.

Others assume that a patient's lung cancer was caused by smoking, even if he or she had stopped smoking years ago.

The relationship of lung cancer stigma with time to seek help in days (Lg10) was then examined by sex of the participants on the total CLCSS scores and all subscales. There was no significant relationship between time to seek help in days (Lg10) and lung cancer stigma, as measured by the total CLCSS ( $r$ $=.20, p=.241)$, or the four subscales: stigma and shame subscale $(r=.19, p=$ $.280)$; social isolation subscale $(r=.05, p=.780)$; discrimination subscale $(r=$ $.22, p=.196)$; or, smoking subscale $(r=.25, p=.145)$ for males. To the contrary, in females, there was a significant relationship between time to seek help in days (Lg10) and lung cancer stigma, on the total CLCSS $(r=.30, p=.023)$, the stigma 
and shame subscale $(r=.30, p=.024)$, and the social isolation subscale $(r=.31$, $p=.020)$. For female participants, there was no significant relationship between time to seek help in days $(\operatorname{Lg} 10)$ and the discrimination subscale $(r=.16, p=$ $.234)$ or the smoking subscale $(r=.22, p=.095)$.

Additional analyses were performed examining lung cancer stigma as measured using the total CLCSS score. Participants were divided into three groups according to time to seek help in days for analysis (Group 1: 30 days or less; Group 2: 31 to 90 days; Group 3: 91 days or greater). There was no significant difference across the groups on the mean total scale score or the means scores for two of the four subscales: social isolation and discrimination. There was a significant difference at the $p<.05$ level on the stigma and shame subscale for two of the groups: $F(2,90)=3.52, p<.05$. The actual difference in mean scores between Group 2 ( 31 to 90 days) and Group 1 (less than 30 days) was fairly small revealing individuals who waited 31 to 90 days to seek help for lung cancer symptoms scored slightly higher on the stigma and shame subscale items than individuals who sought help within 30 days of symptom awareness. Post-hoc comparisons using the Tukey HSD test indicated a mean score for Group $2(M=24.70, S D=5.24)$ and Group $1(M=22.28, S D=3.84)$. The effect size, calculated using eta squared, was .07 and is considered a medium effect size (Stevens, 2009). For the smoking subscale, there was a significant difference for the same two groups as well: $F(2,90)=4.00, p<.05$. However, the actual difference in mean scores between Group 2 (31 to 90 days) and Group 1 (30 days or less) was fairly small. Calculation of eta squared revealed a 
medium effect size of .08. Post-hoc comparisons using the Tukey HSD test indicated that the mean score for Group $2(M=15.70, S D=2.58)$ was significantly different from Group $1(M=13.59, S D=3.18)$ indicating that individuals who waited 31 to 90 days to seek help for lung cancer symptoms scored slightly higher on the smoking subscale items than individuals who sought help within 30 days of symptom awareness. Group 3 (91 days or greater) $(M=$ $14.54, S D=3.01)$ did not differ significantly from either Group 1 or Group 2.

Pearson product moment correlations between stigma and time to seek help were run by ethnicity. There was a significant relationship between time to seek help in days (Lg10) and lung cancer stigma as measured by the total $\operatorname{CLCSS}(r=.28, p=.016)$, stigma and shame subscale $(r=.26, p=.021)$, and smoking subscale $(r=.28, p=.013)$ for Caucasian participants. However, there was no significant relationship between time to seek help and the total CLCSS or any of the four subscales in African-American participants.

\section{Smoking Status}

For the categorical independent variable, smoking status, and time to seek help in days (Lg10), a one-way between-groups analysis of variance was performed. Group 1 represented never smokers; Group 2 represented former smokers; and Group 3 represented current smokers at the time of their lung cancer diagnosis. There was no significant difference in mean time to seek help in days $(\operatorname{Lg} 10)$ across the three groups $(F(2,90)=1.529, p=.222)$.

To further explore the influence of smoking status on the time to seek help in days, a Kruskal-Wallis $\mathrm{H}$ Test was performed, and there was no significant 
association between smoking status and the three time frames to seek help groups (Group 1, 30 days or less; Group 2, 31 to 90 days; Group 3, 91 days or

greater). This finding was similar when the data were split on ethnicity and a oneway between-groups analysis of variance was performed on time to seek help in days (Lg10) and smoking status for Caucasians $(F(2,73)=.860, p=.427)$ versus African-Americans $(F(2,13)=.471, p=.635)$. The relationship between smoking status and time to seek help in days (Lg10) through one-way betweengroups analysis of variance was also evaluated by sex. There was no significant relationship by sex of the participant.

\section{Partial Correlations}

Partial correlations are similar to Pearson product moment correlations, except they allow you to control for an additional variable to get a more accurate indication of the relationship between two variables if you suspect another variable may be confounding the results (Pallant, 2010). Therefore, to further examine the relationship between the independent variables and the dependent variable, partial correlation was used to explore the relationship for one independent variable while controlling for the other independent variable. Preliminary analyses were performed for all partial correlations to ensure no violation of the assumptions of normality, linearity and homoscedasticity.

\section{Healthcare System Distrust}

The relationship between healthcare system distrust (as measured by the RHCSDS) and time to seek help in days (Lg10) was explored while controlling for total lung cancer stigma scores on the CLCSS. There was a weak, positive, 
partial correlation between healthcare system distrust and time to seek help in days (Lg10) for symptoms suggestive of lung cancer, controlling for lung cancer stigma, $r=.27, n=89, p<.01$, with higher levels of healthcare system distrust being associated with greater number of days to seek help for symptoms suggestive of lung cancer. An inspection of the zero order correlation $(r=.08)$ suggested that controlling for lung cancer stigma had a significant effect on the strength of the relationship between the two variables. Previous statistical analyses of healthcare system distrust and the dependent variable did not reveal a statistically significant result. Partial correlation results suggest healthcare system distrust may influence time to seek help in lung cancer when lung cancer stigma is controlled in the analyses.

\section{Lung Cancer Stigma}

Partial correlation was also used to explore the relationship between lung cancer stigma and time to seek help in days (Lg10), while controlling for healthcare system distrust. There was a modest, positive, partial correlation between lung cancer stigma and time to seek help in days (Lg10) for symptoms suggestive of lung cancer, controlling for healthcare system distrust, $r=.37, n=$ $89, p<.0001$, with higher levels of lung cancer stigma being associated with a greater number of days to seek help for symptoms suggestive of lung cancer. An inspection of the zero order correlation $(r=.27)$ suggested that controlling for healthcare system distrust had a significant effect on the strength of the relationship between the two variables. 
Partial correlation was then used to explore the relationship between lung cancer stigma and time to seek help in days (Lg10), while controlling for scores on the Modified Marlowe-Crowne Social Desirability Scale. There was a weak, positive, partial correlation between lung cancer stigma and time to seek help in days (Lg10) for symptoms suggestive of lung cancer, controlling for social desirability, $r=.27, n=90, p<.05$, with high levels of lung cancer stigma being associated with greater number of days to seek help for symptoms suggestive of lung cancer. An inspection of the zero order correlation $(r=.27)$ suggested that controlling for social desirable responding had little effect on the strength of the relationship between these two variables.

To explore another potential confounding variable, age at lung cancer diagnosis, on the relationship between the independent variables and the dependent variable, partial correlation was performed. For lung cancer stigma and time to seek help in days (Lg10), there was a small, positive, partial correlation between lung cancer stigma and time to seek help in days (Lg10), controlling for age at lung cancer diagnosis, $r=.27, n=90, p<.05$, with higher levels of lung cancer stigma being associated with greater number of days to seek help for symptoms suggestive of lung cancer. However, an inspection of the zero order correlation $(r=.27)$ suggested that controlling for age at lung cancer diagnosis had little effect on the strength of the relationship between these two variables. Partial correlation was then used to explore the relationship between healthcare system distrust and time to seek help in days (Lg10), controlling for social desirability finding no statistical significance. There was also no statistical 
significance noted between healthcare system distrust and time to seek help in days (Lg10), controlling for age at lung cancer diagnosis.

\section{Relationship of Potential Confounding Variables and Healthcare System \\ Distrust, Lung Cancer Stigma, Smoking Status, and Time to Seek Help in Symptoms Suggestive of Lung Cancer}

The second specific aim of the study focused on examining the bivariate relationships of potential confounding variables (socioeconomic status, ethnicity, and social desirability) and the independent variables and dependent variable. To address this aim, one-way analyses of variance were conducted. Preliminary analyses were performed to ensure no violation of assumptions of normality, linearity, and homoscedasticity. Tests of differences in the means between socioeconomic status (as measured by annual income and perceived financial status) and ethnicity and the independent variables (healthcare system distrust, lung cancer stigma, and smoking status) and the dependent variable (time to seek help in days [Lg10]) were performed. Finally, tests of differences in the means were conducted on the dependent variable (time to seek help in days grouped in 3 groups) and independent variables, healthcare system distrust and lung cancer stigma. For completeness, other potential confounding demographic variables (lung cancer stage at diagnosis, marital status, sex, employment status, insurance status, smoking status, and education level) were also examined using one-way analysis of variance. 


\section{Socioeconomic Status}

Socioeconomic status was measured by annual income at diagnosis and perceived financial status at diagnosis. Annual income was reported in three categories: less than $\$ 25,000 ; \$ 25,000$ to $\$ 50,000$; and, greater than $\$ 50,000$. However, annual income may not be reflective of one's perception of their socioeconomic status. Therefore, a second measure assessed the participants' perceived financial status. This was reported in three categories: I have more than I need to live well; I have just about enough to get by; and I sometimes struggle to make ends meet.

A one-way between-groups analysis of variance was conducted to explore the impact of socioeconomic status as measured by perceived financial status on healthcare system distrust. Group 1 reflected participants who answered 'I have more than I need to live well'; Group 2 reflected participants who answered 'I have just about enough to get by'; and, Group 3 reflected participants who answered 'I sometimes struggle to make ends meet'. Mean healthcare system distrust scores differed for the three perceived financial status groups: $F(2,89)=$ 4.6, $p=.01$. The effect size, calculated using eta squared, was .09. Post-hoc comparisons using the Tukey HSD test indicated that the mean score for Group $1(M=33.71, S D=5.22)$ was significantly different from both Group $2(M=$ 29.51, $S D=6.42)$ and Group $3(M=28.92, S D=5.34)$ indicating that participants with higher perceived levels of financial status had higher levels of total distrust scale scores than participants with middle range and lower perceived financial status. Lung cancer stigma was then examined through a one-way between- 
groups analysis of variance by socioeconomic status (as measured by perceived financial status). Mean lung cancer stigma scores did not differ by group. The association of perceived financial status and smoking status was examined using Pearson Chi-square test for independence (see Table 4.6 for Chi-square tests of independence between categorical covariates and smoking status). There was no significant association between the two variables; $X^{2}(4, \mathrm{n}=93)=5.221, p=$ .265 , phi $=.265)$. Finally, to evaluate the whether time to seek help varied by perceived financial status, a one-way between-groups analysis of variance was conducted. Mean time to seek help did not differ across financial status levels.

The bivariate relationship between annual income and time to seek help was then evaluated. Group 1 represented study participants making annual household incomes of greater than $\$ 50,000$; Group 2 represented study participants making $\$ 25,000$ - $\$ 50,000$; and, Group 3 represented study participants making annual household incomes of less than $\$ 25,000$. Healthcare system distrust was examined through a one-way between-groups analysis of variance, and there were no differences in healthcare system distrust scores among the three groups. Lung cancer stigma was then examined through a oneway between-groups analysis of variance for annual income. There were no significant differences in mean lung cancer stigma scores among the three groups. The association of annual income and smoking status was evaluated with a Pearson Chi-square test for independence. However, findings did not support a significant association between the two variables; $X^{2}(4, N=93)=$ 1.693, $p=.792, p h i=.135)$. Finally, to evaluate potential differences in mean 
time to seek help by annual income, a one-way between-groups analysis of variance was conducted and there was no significance.

\section{Ethnicity}

Ethnicity was measured as a demographic item on the THSBDQ. There were six categories for each participant to self-report ethnicity (Caucasian, African-American, Asian, Native Hawaiian or Other Pacific Islander, American Indian or Alaska Native, and Hispanic). The participants ( $=93)$ represented three of the six identified categories: $81.7 \%$ Caucasian, $17.2 \%$ African-American, and 1.1\% Hispanic. Since there was only one individual self-identified as Hispanic and an $n$ of one is not appropriate for delineation of a group for statistical purposes, two groups were delineated in the statistical analyses. Group 1 represented Caucasian study participants, and Group 2 represented AfricanAmerican study participants.

To examine potential differences in mean healthcare system distrust by ethnicity, a one-way between-groups analysis of variance was performed and did not reveal a significant difference in mean healthcare system distrust scores between the Caucasian and African-American study participants. This is not to suggest the nonexistence of healthcare system distrust as the mean scores and standard deviation on the RHCSDS for Caucasians was $30.75+6.32$ and for African-Americans was $28.38+5.10$. RHCSDS total scores range from 9 to 45 with higher scores reflecting increased levels of healthcare system distrust. 
Lung cancer stigma (as measured by the CLCSS) and ethnicity was then examined using one-way between-groups analysis of variance. There was a statistically significant difference at the $p<.05$ level in lung cancer stigma scores for Group 1 (Caucasian) and Group 2 (African-Americans): $F(2,90)=3.414, p<$ .05. The effect size, calculated using eta squared, was .07 and is considered a medium effect size. Post-hoc comparisons using the Tukey HSD test and visual examination of the mean scores revealed that the mean score for Group 2 (African-Americans; $M=75.1, S D=12.46$ ) was significantly difference from Group 2 (Caucasians; $M=67.2, S D=10.93$ ) indicating that African-Americans had higher levels of lung cancer stigma scale scores than Caucasians. Refer to Table 4.6 for differences in means between categorical covariates and continuous independent variables and the dependent variable. 
Table 4.6

Differences in the Means between Categorical Covariates and Continuous Independent Variables and the Dependent Variable

\section{Comparison}

Annual Income \&

Healthcare System

Distrust

Annual Income \& Lung

Cancer Stigma

Annual Income \& Time

to Seek Help

Perceived Financial

Status \& Healthcare

System Distrust

Perceived Financial

Status \& Lung Cancer

Stigma

Perceived Financial

Status \& Time to Seek

Help

Ethnicity \& Healthcare

System Distrust

Ethnicity \& Lung

Cancer Stigma

Ethnicity \& Time to

Seek Help
F-statistic

(df)

p

.73

2, 89

1.20

2, 90

.31

.97

2, 90

.38

4.60

2, 89

$.01^{*}$

2.56

2, 90

.08

2.08

2, 90

1.01

2, 89

.37

3.41

2,90

$.04^{*}$

${ }^{*} p<.05$

2, 90

.36 
To evaluate the association between ethnicity and the categorical independent variable, smoking status, a Pearson Chi-square test for independence was performed (see Table 4.7 for Chi-square analysis between categorical covariates and smoking status) and indicated no significant association between the two variables; $X^{2}(4, N=93)=4.682, p=.321$, phi $=$ .224). A one-way between-groups analysis of variance was then performed on ethnicity on the dependent variable, time to seek help in days (Lg10) and did not reveal a statistically significant difference in time to seek help in days (Lg10) between the Caucasians and African-Americans. 


\section{Table 4.7}

Chi-square Test for Independence between Covariates (Categorical Variables) and Categorical Independent Variable (Smoking Status)

Variable

Socioeconomic Status:

Annual Income

Perceived Financial Status

Ethnicity
Smoking Status

Chi-square (df,n), alpha, phi coefficient

\section{Social Desirability}

Social desirability was evaluated as a potential confounding variable because of the sensitive nature of the topic of this study; distrust and stigma. A one-way between-groups analysis of variance was performed on smoking status and social desirability, measured by the Modified Marlowe-Crowne Social Desirability Scale. There was no statistically significant difference in social desirability scores between the three groups. The relationship between healthcare system distrust and social desirability was then investigated using Pearson product moment correlation coefficient. There was not a statistically significant relationship between these two variables. Lung cancer stigma was then examined in relation to social desirability using Pearson product moment correlation coefficient, and there was a small, negative correlation between the two variables, $r=-.23, n=93, p<.05$, with high levels of social desirability associated with lower levels of reported lung cancer stigma. Finally, the 
relationship between the dependent variable, time to seek help in days (Lg10), and social desirability was investigated using Pearson product moment correlation coefficient. A statistically significant relationship was not supported between time to seek help and social desirability.

\section{Dependent Variable and Demographic Variables}

As mentioned earlier, other potential confounding demographic variables (lung cancer stage at diagnosis, marital status, gender, employment status, insurance status, smoking status, and education level) were examined using one-way analysis of variance to explore their impact on the dependent variable, time to seek help in days ( $\operatorname{Lg} 10)$. With the exception of education level, none of the analyses reached statistical significance to indicate a difference in mean scores (see Table 4.8). Concerning education, statistical significance was noted. However, the test noted a violation of the assumption of homogeneity of variance. 
Table 4.8

Differences in the Means of Time to Seek Help in Days by Demographic Characteristics

\begin{tabular}{lccc}
\hline Characteristic & F-statistic & (df) & $\mathbf{p}$ \\
Lung Cancer Stage & .033 & 3,88 & .992 \\
Marital Status & 1.316 & 3,88 & .274 \\
Sex & .095 & 1,90 & .758 \\
Ethnicity & 1.037 & 2,90 & .359 \\
Employment Status & .835 & 4,87 & .507 \\
Insurance Status & 1.060 & 3,88 & .370 \\
Smoking Status & 1.942 & 2,89 & .149 \\
Education Level & 3.223 & 3,88 & $.026^{*}$ \\
\end{tabular}

${ }^{*} p<.05$

\section{Multivariable Regression}

Hierarchical linear regression was used to assess the ability the total healthcare system distrust score, the total lung cancer stigma score, and smoking status to predict timing of help-seeking behavior in lung cancer, after controlling for the influence of social desirability, ethnicity, annual income, and perceived financial status. Preliminary analyses were conducted to ensure no violation of the assumptions of normality, linearity, multicollinearity and homoscedasticity. Social desirability, ethnicity, annual income, and perceived financial status were entered at Step 1, and explained $10 \%$ of the variance in time to seek help in lung cancer. After entry of the total healthcare system 
distrust scores, total lung cancer stigma scores, and smoking status at Step 2, the total variance explained by the model as a whole was $23 \%, F(7,84)=3.591$, $p<.01$. In the final model, total lung cancer stigma scale scores had a beta value of $.44, p<.001$; total healthcare system distrust had a beta value of $.25, p<.05$; ethnicity with a beta value of $.22, p=.05$; and perceived financial status with a beta value of $-.31, p=.03$ were statistically significant. See Table 4.9 for a summary of the hierarchical linear regression analysis. 
Table 4.9

Summary of Hierarchical Regression Analysis for Variables Predicting Time to Seek Help in Days (Lg10) in Lung Cancer ( $N=93)$

\begin{tabular}{|c|c|c|c|c|c|c|c|c|}
\hline Variables & $\begin{array}{c}\text { Step } \\
\mathrm{F}\end{array}$ & $\begin{array}{l}\text { Step } \\
P \\
\text { value }\end{array}$ & $\mathrm{R}^{2}$ & $\begin{array}{l}\text { Adj } \\
\mathrm{R}^{2}\end{array}$ & $\begin{array}{l}F \text { for } R^{2} \\
\text { change }\end{array}$ & $\begin{array}{c}P \\
\text { value } \\
\text { for } R^{2} \\
\text { change }\end{array}$ & $\begin{array}{c}\text { Standardized } \\
\beta\end{array}$ & $\begin{array}{c}\beta \\
P \\
\text { value }\end{array}$ \\
\hline $\begin{array}{l}\text { Step } 1 \\
\text { Perceived } \\
\text { Financial Status } \\
\text { Annual Income } \\
\text { Ethnicity } \\
\text { Social Desirability }\end{array}$ & 2.43 & .054 & .10 & .06 & 2.43 & .054 & $\begin{array}{r}-.31 \\
-.02 \\
.26 \\
.00\end{array}$ & $\begin{array}{l}.029 \\
.92 \\
.028 \\
.998\end{array}$ \\
\hline $\begin{array}{l}\text { Step } 2 \\
\text { Perceived } \\
\text { Financial Status } \\
\text { Annual Income } \\
\text { Ethnicity } \\
\text { Social Desirability } \\
\text { Smoking Status } \\
\text { HCS Distrust } \\
\text { Lung Cancer } \\
\text { Stigma }\end{array}$ & 3.59 & .002 & .23 & .17 & 4.73 & .004 & $\begin{array}{r}-.31 \\
.02 \\
.22 \\
.06 \\
.03 \\
.25 \\
\\
.44\end{array}$ & $\begin{array}{l}.030 \\
.864 \\
.049 \\
.539 \\
.765 \\
.037 \\
.000\end{array}$ \\
\hline
\end{tabular}

Note: HCS Distrust = Healthcare System Distrust

Support for the overall hypothesis that greater healthcare system distrust, higher levels of self-perceived lung cancer stigma, and positive smoking status predict increased time from symptom onset to help-seeking, controlling social desirability, socioeconomic status, and ethnicity is conflicting. While greater healthcare system distrust and higher levels of self-perceived lung cancer stigma predict increased time from symptom onset to help-seeking, a positive smoking status is not predictive of time to seek help. 


\section{Summary}

Multiple statistical analyses were performed to examine the relationship between the independent variables and the dependent variable, time to seek help in lung cancer. Significant findings included a small positive partial correlation between healthcare system distrust and time to seek help in lung cancer if controlling for the other independent variable, lung cancer stigma. There were similar findings when partial correlation was used to examine lung cancer stigma and time to seek help in lung cancer when healthcare system distrust was controlled. There was a positive correlation between higher lung cancer stigma scores and time to seek help in lung cancer. Further when the data set was split by gender, a positive correlation between lung cancer stigma and time to seek help in lung cancer was noted in females but not males. The data set was also split by ethnicity, and a positive correlation was noted between lung cancer stigma and time to seek help in Caucasians but not African-Americans. Finally, smoking status did not reveal a statistically supported relationship with the dependent variable in any of the analyses.

Significant findings from an examination of the confounding variables with the independent variables and dependent variable revealed that socioeconomic status (as measured by annual income) was not statistically related. However, higher socioeconomic status (as measured by perceived financial status) had higher total healthcare system distrust scores. For total lung cancer stigma scores, African-Americans had higher total scale scores than Caucasians. For 
social desirability, there was a small negative correlation between socially desirable responding and total lung cancer stigma scale scores.

Hierarchical linear regression revealed that two of the three hypothesized predictor variables (healthcare system distrust, lung cancer stigma) accounted for $13 \%$ of the variance in time to seek help in the model. In the final model, the control variables explained $10 \%$ of the variance in time to seek help, while the remaining variables accounted for $13 \%$, bringing the total explained variance to $23 \%$. Each of the following was uniquely associated with time to seek help in the final model: ethnicity, socioeconomic status measured by perceived financial status, healthcare system distrust, and lung cancer stigma. 


\section{CHAPTER FIVE}

DISCUSSION

\section{Introduction}

The purposes of this chapter are to (a) provide a summary of the study, (b) discuss the importance of the findings and implications from the study, (c) present recommendations for future research based upon the findings, and (d) address the limitations and strengths of the study. The chapter is divided into four sections based upon these purposes followed by an overall conclusion.

\section{Summary of the Study}

Lung cancer kills more people than any other cancer worldwide primarily because it is often diagnosed at an advanced stage (Ferley et al., 2010; Jemel et al., 2011; SEER Program, 2011). Some people have symptoms suggestive of lung cancer, but delay seeking help for those symptoms. Delayed help-seeking behavior increases the morbidity and mortality in individuals with lung cancer (Corner et al., 2006; Tod et al., 2008; Tod \& Joanne, 2010). The purpose of this research was to examine the influence of healthcare system distrust, lung cancer stigma, and smoking status on the timing of help-seeking behavior in symptoms suggestive of lung cancer. Lung cancer patients were the targeted population for this study, as they were able to retrospectively provide an understanding of the 
timing from the first symptom experienced to the first time help was sought for those symptoms as well as insight into potential variables that could be influential on the timing to seek help in lung cancer.

The following specific aims were the focus of this study:

Aim 1. To explore the influences of healthcare system distrust, lung cancer stigma, and smoking status on the timing of help-seeking behavior in individuals with symptoms suggestive of lung cancer.

Aim 2. To examine the bivariate relationships of socioeconomic status, ethnicity, and social desirability as related to healthcare system distrust, lung cancer stigma, smoking status, and timing of help-seeking behavior.

Aim 3. To determine the predictive power of the model for timing in helpseeking behavior in lung cancer patients.

The research hypothesis of the study was:

Greater healthcare system distrust, higher levels of self-perceived lung cancer stigma, and positive smoking status predict increased time from symptom onset to help-seeking, controlling social desirability, socioeconomic status, and ethnicity.

A conceptual model (Figure 1.2, p. 20) was developed to provide the framework for assessing help-seeking behavior in individuals with symptoms suggestive of lung cancer. This study explored four of the eight constructs of the model: (a) sociodemographic factors, (b) healthcare system distrust, (c) lung cancer stigma and, (d) symptom appraisal. The study employed a descriptive, cross-sectional design using questionnaires that were administered via an in- 
person interview to individuals diagnosed with lung cancer at two hospital-based study sites. Primary study outcomes evaluated the timing of help-seeking behavior (via an investigator-developed questionnaire exploring the time to seek help in lung cancer and demographic information), healthcare system distrust (via the Revised Healthcare System Distrust Scale), lung cancer stigma (via the Cataldo Lung Cancer Stigma Scale), and smoking status. Data analysis was performed via SPSS Version 20.0 and descriptive and inferential analyses were performed. Specifically, frequencies, means, standard errors, and ranges on all variables were examined. Bivariate relationships of the independent and dependent variables were analyzed with Pearson product moment correlation coefficients and Chi-square tests for independence. Finally, hierarchical regression analysis was used to assess the ability of healthcare system distrust, lung cancer stigma, and smoking status to predict timing of help-seeking behavior in lung cancer.

\section{Discussion and Implications}

Sample characteristics, symptoms, and instruments will be discussed followed by the findings related to the specific aims and hypothesis.

\section{Sample Characteristics}

The primary outcome of interest was time to seek help for symptoms suggestive of lung cancer. The mean number of days that a participant reported waiting to seek help for their lung cancer symptoms was 70 days. However, time to seek help was fairly equally distributed across three time categories with $42 \%$ seeking help in 30 days or less, $29 \%$ seeking help in 31 to 90 days, and $29 \%$ 
seeking help in greater than 90 days. Lung cancer is associated with a high mortality rate when advanced. The five-year survival rates for stage IIIA, IIIB, and IV lung cancer are only $14 \%, 5 \%$ and $1 \%$ respectively (ACS, 2013). Although patient delay after symptom awareness has been defined as waiting to seek help for three months or longer (Facione, 1993; Pack \& Gallo, 1938), with the high mortality rate that is associated with lung cancer as it advances, the arbitrary delineation of three months may not be appropriate in this disease. Nearly half $(47.3 \%)$ of the participants that waited one month or longer to seek help for their symptoms were diagnosed with advanced stage (stage IIIA/B or IV) lung cancer. Although waiting one month or more to seek help for lung cancer symptoms in this study cannot be causally linked to advanced stage lung cancer, the act of waiting to seek help with symptoms suggestive of lung cancer is concerning. Lung cancer symptoms should be evaluated without haste. In lung cancer, patient delay for the purposes of research should be defined at one month or greater as opposed to three months.

In this sample, the mean age at diagnosis was 62 years which is slightly younger than the majority of individuals diagnosed with lung cancer. The American Cancer Society (2013) notes the average age of a lung cancer patient at diagnosis is 71 years. While the majority of individuals with lung cancer are 65 years or older when first diagnosed (ACS, 2013), the majority of the sample (81\%) was 55 years or older. The younger age found in this sample may be reflective of the higher percentage of smokers geographically. While the Center for Disease Control (2012) notes a national smoking rate of $21 \%$ for adults age 
18 or older, Kentucky has a $29 \%$ smoking rate. For young adults in Kentucky age 18 to 24 years old, the smoking rate is $31.7 \%$ compared to the national smoking rate of $18.9 \%$ in this age group (CDC, 2012). Kentucky also has a significant number of teenage smokers with $15.9 \%$ smokers among adolescent youth age 12 to 17 years. This is higher than the national smoking rate of $10.2 \%$ among adolescent youth (CDC, 2012).

Nearly two-thirds (62.4\%) of the participants were women which is higher than the national average. Lung cancer is fairly equally dispersed between genders with approximately $48 \%$ of new lung cancer cases in women and $52 \%$ in men (ACS, 2013). One reason the sample may have more women may be related to the dramatic increase in the number of women who smoke over the past few decades (American Society of Clinical Oncology, 2013). This is significant when considering potential gender differences in help-seeking behavior in lung cancer symptoms.

Of the total study sample, $81.7 \%$ were Caucasian and $17.2 \%$ were African-American which is fairly reflective of the population characteristics of the geographic area (74.3\% Caucasian and $21.0 \%$ African-American) per the most recent U. S. Census Bureau statistics (2013) for Kentucky. Although AfricanAmerican and Caucasian women have a similar disposition for developing lung cancer, African-American men are $40 \%$ more likely to develop lung cancer than their Caucasian counterpart. However, only one study participant in this sample was an African-American male. This limits the generalizability of the findings to African-American men with lung cancer. 
There are several sample characteristics that may have influenced the findings. First, more than one-third (35.5\%) of the participants had a college education or higher. While a college education does not equate to knowledge of lung cancer symptoms, it does suggest an exposure to a variety of formal educational experiences. Alternatively, some participants may have increased symptom knowledge and may have sought help earlier. Another characteristic of importance was most participants were married (64.5\%). Studies have reported that individuals were more likely to seek help when symptoms were either disclosed or witnessed by a close friend or family member such as a spouse (Bish et al., 2005; Griffith et al., 2011). The high percentage of married individuals in this study may have positively impacted the time to seek help in lung cancer symptoms.

Another relevant sample characteristic was access to healthcare. Insurance provides access to healthcare by removing a financial barrier in many cases. Access to healthcare can play an important role in help-seeking behavior (Bish et al., 2005). The majority of the participants had some form of insurance (87.1\%). Thus, when access to healthcare is considered in the context of helpseeking behavior and a positive insurance status is noted, decreased time to seek help is the expected behavior. In this study, there was no difference in the mean scores between insurance status and time to seek help.

Lung cancer has been strongly associated with smoking (Cataldo et al., 2012) and is the greatest risk factor for the development of lung cancer (ACS, 2013). However, the percentage of never smokers in this sample was 
considerably higher (32.3\%) than the reported national average (10 to 15\%; Thun et al., 2006). In fact, this sample reflected a fairly equal proportion of past smokers $(35.5 \%)$, never smokers $(32.3 \%)$, and current smokers $(32.3 \%)$. In addition, slightly greater than two-thirds of the sample (67.7\%) were not smokers at diagnosis. While the larger percentage of never smokers is uncharacteristic of the population of lung cancer patients, it provides a unique opportunity to understand help-seeking behavior in an under-researched segment of the lung cancer population. One potential explanation of why there were more never smokers in this sample compared to the national average of never smokers with lung cancer may be linked to the higher percentage of smokers in Kentucky. As mentioned previously, Kentucky has a $29 \%$ smoking rate (CDC, 2012) which is higher than the national rate. With a large amount of tobacco smokers, there is a greater potential for never smokers to be exposed to greater amounts of secondhand smoke in this area.

One plausible explanation for the differences in the characteristics of this sample compared to the national characteristics of the average lung cancer patients is high levels of radon exposure. Exposure to radon is the second leading cause of lung cancer (ACS, 2013; Sethi, El-Ghamry, \& Kloecker, 2012). This study was conducted in Kentucky, an area known for its high levels of radon. Data were not collected on the specific location of participants' residences therefore the amount of radon exposure was unknown. It is likely, however, that many participants had significant radon exposure during their lives. This high level of radon exposure may account for the development of lung cancer in a 
higher number of younger individuals, a greater number of women and in a larger number of never smokers.

Tobacco use and prolonged secondhand smoke exposure are other factors that increase an individual's risk for the development of lung cancer (ACS, 2013). Tobacco use, in particular, is associated with the stigma surrounding lung cancer (Lebel \& Devins, 2008). Stigma may influence helpseeking behavior. Addressing the stigma associated with lung cancer in the population of never smokers clearly presents new challenges that require different approaches from the population of lung cancer patients that are current or former smokers. There are studies that propose lung cancer stigma does not differ in lung cancer patients with a smoking history compared to never smokers (Cataldo et al., 2012). However, non-smokers and smokers alike many times experience negative attitudes about their lung cancer diagnosis (Raleigh, 2010). This is important to consider when interpreting the results related to stigma in lung cancer patients regarding smoking history.

\section{Symptoms}

Symptoms are indicators that lead people to seek healthcare. In lung cancer, the American Cancer Society (2013) reports that cough, dyspnea, and fatigue are the most common symptoms. This study supports these findings and found the most common symptoms reported by participants were cough and respiratory symptoms $(47.3 \%)$ and fatigue $(22.6 \%)$. With individuals with a history of tobacco use, cough was described as a different cough than previously experienced secondary to smoking. In addition, several studies suggest that lung 
cancer patients present with symptom clusters. Every participant reported at least two symptoms when interviewed and nearly one-fourth $(22.6 \%)$ reported experiencing three symptoms at diagnosis. This supports several studies that note the presence of multiple symptoms at diagnosis in lung cancer as opposed to a solitary symptom (Brown et al., 2011; Henoch et al., 2009; Sarna \& Brecht, 1997).

\section{Instruments}

This study used two Likert-type scales: the Revised Health Care System Distrust Scale (RHCSDS) and the Cataldo Lung Cancer Stigma Scale (CLCSS) to measure two of the independent variables. Psychometric testing of the RHCSDS revealed a strong internal consistency with a Cronbach's coefficient alpha of .91. In the original psychometric testing of the RHCSDS, a Cronbach's coefficient alpha of .85 was obtained in a sample of 246 participants. The ethnic composition of the original study was different from this study with $56 \%$ AfricanAmerican, 36\% Caucasian, and 8\% from other ethnic groups (Shea et al., 2008) which may affect comparing results. This is a short nine-item scale that was easy to administer and is a dual-dimensional measurement that assesses values and competence in the healthcare system.

Psychometric testing of the CLCSS also revealed a strong internal consistency with a Cronbach's coefficient alpha of .95. In the original psychometric study of the CLCSS, internal consistency reliability yielded a Cronbach's coefficient alpha of .96 in a sample of 186 lung cancer patients (Cataldo et al., 2011). While the Cronbach's coefficient alpha of .95 in this study 
is strong, the high Cronbach's coefficient alpha may indicate there is some level of redundancy in a 31-item scale. The scale is comprised of four subscales. In practical application, the researcher received several requests from participants for verbal clarification. Many participants reported that several scale items seemed similar during the data collection period.

\section{Aim 1: Influence of Healthcare System Distrust, Lung Cancer Stigma, and Smoking Status on Time to Seek Help in Lung Cancer}

Healthcare system distrust and time to seek help in lung cancer.

Distrust is an important barrier to perceived access to healthcare (Katapodi et al., 2010; McAlearney et al., 2012; Shea et al., 2008; Yang, Matthews, \& Hillemeier, 2011). The overall mean distrust score for this sample was 30.37 on a scale that ranged from 9 (low level of distrust) to 45 (high level of distrust). The findings revealed a moderate level of healthcare system distrust overall in this sample. Although time to seek help and healthcare system distrust have not been studied together, it was plausible that healthcare system distrust would be correlated to greater time to seek help in symptoms suggestive of lung cancer. However, this was not the case in this sample.

Previous studies that have explored healthcare system distrust have focused on ethnic differences. Higher levels of healthcare system distrust have been found in two recent studies noting African-Americans reported higher levels of healthcare system distrust (Armstrong et al., 2012; Armstrong et al., 2013) when compared to Caucasians. Time to seek healthcare was not the focus of either of these studies. One study focused on the influence of healthcare system 
distrust on the willingness to undergo genetic testing (Armstrong et al., 2012). The other study focused on racial discrimination as an explanation for higher levels of African-American healthcare system distrust (Armstrong et al., 2013). The finding of higher healthcare system distrust scores in African-Americans compared to Caucasians was not supported in this study. To the contrary, Caucasian participants in this study scored slightly higher on the total RHCSDS scores $(30.75+6.32)$ than African-American participants $(28.38+5.10)$. This was an unexpected finding as previous studies have found a higher level of healthcare system distrust in African-Americans compared to Caucasian individuals (Armstrong et al.,2007; Armstrong et al., 2008; Armstrong et al., 2012; Armstrong et al., 2013; Dovidio et al., 2008; Katapodi et al., 2010; Russell et al., 2012). However, the previous studies were focused on ethnic differences and had higher percentages of African-Americans than this study.

For this study, healthcare system distrust was examined as a potential influential variable on time to seek help in symptoms suggestive of lung cancer. In addition to analyzing the data by ethnicity, the data were analyzed by correlation and one-way analysis of variance. First, healthcare system distrust was not correlated to time to seek help in days $(r=.07, p=.532)$. Second, when study participant responses were grouped into three time periods (less than 30 days, 31 to 90 days, and greater than 90 days), there was not a statistically significant difference in healthcare system distrust scores and time to seek help. Anecdotally, in the course of interviewing participants during the Timing of Help-Seeking Behavior and Demographic Questionnaire (THSBDQ) portion of 
data collection, several participants described developing a sense of healthcare system distrust after initially seeking help for their lung cancer symptoms. These individual accounts described seeking help promptly but experiencing delays within the healthcare system primarily from their primary care provider. This led to a dissatisfaction in their overall care and a subsequent perception of technical incompetence of their healthcare provider. These individuals described their development of distrust of the healthcare system as a reflection of this experience because they attributed their advanced stage of lung cancer when they were diagnosed to their provider's delay. Many felt if they were diagnosed when they first sought help for their symptoms, their stage of lung cancer would have been earlier and prognosis would have been drastically different. Some individuals gave examples of being treated with antibiotics for pneumonia without a confirmatory chest radiograph or treated repeatedly for bronchitis with different courses of antibiotics. One female study participant reported shortness of air, cough, and intermittent chest pain and stated her primary care provider attributed her symptoms to anxiety without chest radiograph or low-dose computed tomography. She was eventually diagnosed with stage IV lung cancer.

\section{Lung cancer stigma and time to seek help in lung cancer.}

Lung cancer stigma is a perceived stigma related to the individual's disease process. Berger, Ferrans, and Lashley (2001) note that perceived stigma results in actual or potential social disqualification, limited opportunities, and a negative change in identity. These experiences can lead to physical symptoms and/or a change in self-concept, psychosocial symptoms, avoidance, or 
minimization. The overall mean lung cancer stigma score for this sample was 68.60 on a scale that ranged from 31 (low level of lung cancer stigma) to 124 (high level of lung cancer stigma). The findings revealed a modest level of lung cancer stigma overall in this sample. Therefore, it was plausible for increased lung cancer stigma to be associated with greater time to seek help in symptoms suggestive of lung cancer.

Higher lung cancer stigma scores had a weak positive association with an increased number of days to seek help in lung cancer $(r=.27, p=.01)$. Although there is no specific literature that has examined lung cancer stigma and helpseeking behavior, Chapple et al. (2004) documented the experience of lung cancer patients and fears of related stigma. Blame, fear, and stigmatization influenced the behavioral response of the individual with lung cancer. Although the relationship was weak, any level of lung cancer stigma is important. This finding supports the assertion that lung cancer stigma may lead to delayed helpseeking behavior in lung cancer. Lung cancer patients are more likely to experience shame, self-blame and lower levels of self-esteem (Chapple et al., 2004; Else-Quest et al., 2009; LoConte, Else-Quest, Eickhoff, Hyde, \& Schiller, 2008) related to their lung cancer diagnosis compared to individuals with breast or prostate cancer. These psychological states of shame, self-blame, and poor self-esteem are described in the literature as lung cancer stigma. The following paragraphs will discuss the findings related to lung cancer stigma and time to seek help in detail. 
Lung cancer stigma is a relatively new phenomenon of inquiry. The literature, to date, does not specifically address lung cancer stigma, gender, or ethnicity and their relationship with help-seeking behavior. However, there are studies that have examined the influence of ethnicity and gender in individuals with HIVIAIDS. For example, females exhibited a heightened sense of stigma related to their diagnosis of HIVIAIDS compared to males in a 2008 study (Wolitski et al., 2009). In this study, correlations examining total lung cancer stigma scale scores revealed a relationship between lung cancer stigma and the time to seek help in females but not in males. Given greater stigma in females noted in the HIVIAIDS population and the similarities of the perception of HIVIAIDS and lung cancer (Cataldo et al., 2011; Marlow et al., 2010), this was a plausible finding.

Ethnicity was another influential sociodemographic variable on stigma. In HIV/Hepatitis C co-infected individuals (Lekas, Siegel, \& Leider, 2011), stigma was conceptualized as something that was experienced from multiple stigmatizing statuses (i.e. the HIV diagnosis, coinfection with Hepatitis C, and ethnicity). When lung cancer stigma was analyzed by ethnicity in this study, African-Americans had slightly higher lung cancer stigma scores $(M=75.1, S D=$ 12.46) than Caucasians $(M=67.2, S D=10.93)$. Although African-Americans had higher lung cancer stigma scores, there was no relationship between the lung cancer stigma scores and time to seek help in lung cancer $(r=.113, p=.678)$. Perhaps African-Americans experience a more generalized stigma related to ethnicity and cultural background in everyday situations as opposed to identifying 
the stigma specific to the disease process. This may be a reflection of multiple stigmatizing statuses rooted in racial and social discrimination similar to findings in the HIVIAIDS literature (Henkel, Brown, \& Kalichman, 2008; Lekas et al., 2011). If African-Americans experience a more generalized stigma on a daily basis secondary to racial and social discrimination, lung cancer related stigma may be related to ethnic identity as opposed to lung cancer alone. If a generalized stigma is the etiology of the higher lung cancer stigma scores and is a part of daily life, time to seek help may not be impacted because of the commonplace nature of stigma in the individual's life. On the other hand, lung cancer stigma scores of the Caucasian participants did have a statistically significant correlation to greater time to seek help in lung cancer symptoms ( $r=$ $.28, p<.05)$. This is a weak positive correlation. Perhaps an absence of a generalized stigma secondary to racial and social discrimination reveals a stigma that is associated with the disease process in Caucasian individuals and is reflected in the modest CLCSS scores.

From a different perspective, a one-way between-groups analysis of variance was performed testing total lung cancer stigma scores and the four subscales on time to seek help grouped by time periods (less than 30 days, 31 to 90 days, and greater than 90 days). Although a relationship was not supported on the total CLCSS score, social isolation subscale, or discrimination subscale, individuals who waited 31 to 90 days to seek help scored slightly higher on the stigma and shame subscale as well as the smoking subscale compared to those that sought help in less than 30 days. Individuals with lung cancer frequently talk 
about stigma and related feelings of shame and guilt (Chapple et al., 2004;

LoConte et al., 2008). The findings of higher stigma and shame subscale scores and a delayed time to seek help of one to three months support these findings. Correlations of individual scale items of the CLCSS were analyzed and two stigma and shame subscale items were significant: (a) I feel guilty because I have lung cancer $(r=.23, p=.03)$ and $(b)$ My lung cancer diagnosis was delayed because I put off going to the doctor $(r=.39, p=.00)$. These correlations represented a weak positive and moderate positive relationship respectively. Guilt related to tobacco smoking may influence the stigma associated with lung cancer. Two key scale items of the smoking subscale were also noted to be correlated with greater time to seek help in lung cancer: (a) Lung cancer is viewed as a self-inflicted disease $(r=.20, p=.05)$ and (b) Others assume that a patient's lung cancer was caused by smoking, even if he or she had stopped smoking years ago $(r=.21, p=.04)$. Both correlations represented weak positive relationships.

\section{Smoking status and time to seek help in lung cancer.}

Smoking status was a key demographic variable that was identified from the literature (Cataldo et al., 2011; Stuber et al., 2008). In this study, individuals identified themselves as current smokers, former smokers, or never smokers. A positive smoking status has been associated with delayed help-seeking behavior in individuals with lung cancer secondary to a masking of symptoms or attribution of symptoms to sequela of tobacco smoking (Cataldo et al., 2012; Stuber et al., 2008). Therefore, it was expected that a positive smoking status would be 
correlated to greater time to seek help in symptoms suggestive of lung cancer. However, in this sample, there was no correlation between smoking status and time to seek help. This was an unexpected finding. Perhaps this is simply a reflection of this particular sample, or perhaps there were other variables that were more influential in time to seek help, such as lung cancer stigma, that were more pronounced in this study. In addition, nearly one-third (32.3\%) of the participants were never smokers and this may have factored into the insignificance of the findings of smoking status as related to time to seek help in lung cancer.

\section{Partial correlations of healthcare system distrust and lung cancer}

\section{stigma.}

When the relationship of healthcare system distrust and time to seek help in lung cancer was examined while controlling for lung cancer stigma through partial correlation, there was a statistically significant weak positive relationship ( $r$ $=.272, \mathrm{n}=89, p<.01)$. The partial correlation statistical analysis indicated that higher healthcare system distrust correlated with increased time to seek help in lung cancer. While the initial findings related to healthcare system distrust seem to suggest there is not a correlation with time to seek help in lung cancer, the subsequent findings when lung cancer stigma is controlled for suggest that lung cancer stigma is a fairly significant variable that may be obscuring the understanding of time to seek help. Specifically, healthcare system distrust and lung cancer stigma may frequently coexist in lung cancer patients. 


\section{Aim 2: Examine the Bivariate Relationships of Socioeconomic Status, Ethnicity, and Social Desirability on the Independent Variables and the Dependent Variable}

There are three confounding variables identified in this study: (a) socioeconomic status, (b) ethnicity, and (c) social desirability. Socioeconomic status and ethnicity were measured by demographic questionnaire items on the THSBDQ and the Modified Marlowe-Crowne Social Desirability Scale (Strahan \& Gerbasi, 1972) was used to assess social desirability.

\section{Socioeconomic status.}

Socioeconomic status was measured by two primary means as demographic questions in this study: annual income and perceived financial status at diagnosis. Annual income was self-reported by participants as less than $\$ 25,000, \$ 25,000$ to $\$ 50,000$, or greater than $\$ 50,000$. Perceived financial status was assessed through an income adequacy question. This was reported in three categories: (a) I have more than I need to live well, (b) I have just about enough to get by, and (c) I sometimes struggle to make ends meet. It was important to distinguish socioeconomic status by both annual income as well as perceived financial status to truly capture socioeconomic status from the perception of the individual. While annual income is a commonly reported socioeconomic indicator, annual monetary delimitations may limit a true understanding of socioeconomic status. For example, a single individual that has an annual income of $\$ 50,000$ may have a very different perceived financial status from a family of six with an 
annual income of $\$ 50,000$. Therefore, understanding socioeconomic status from the perceived financial status perspective was critical in this study.

In general, lower socioeconomic status is associated with higher levels of healthcare system distrust (Armstrong et al., 2007; Katapodi et al., 2010). As mentioned in Chapter Four, and contrary to what was hypothesized, the participants in the higher perceived financial status group reported a greater level of healthcare system distrust $(F(2,89)=4.6, p=.01)$ via one-way between groups analysis of variance. There are several conceivable explanations for this finding. For instance, Waljee, Hu, Newman, and Alderman (2008) note that people with greater distrust are generally more vigilant and alert to evaluating outcomes in relation to their healthcare provider. Individuals from a higher socioeconomic background may be more educated and therefore more aware of both the process and the expectations of traversing the healthcare system. This awareness may be reflected in vigilance toward the outcomes of their care thus heightening their distrust. Similarly, individuals from a higher socioeconomic background physically may have more options related to their financial resources. With more options comes the ability to scrutinize the care they are receiving because they have the choice to go elsewhere. The healthcare system is a consumer market and a higher level of financial resources allows the ability to choose healthcare providers, hospitals, and health-related services. The RHCSDS is a dual-dimensional instrument that actually measures values and competence. Perhaps individuals from a higher socioeconomic background are more alert and vigilant to technical competence, which in turn, is reflected in the 
higher scores on the RHCSDS or perhaps dissatisfaction may have been a more accurate description of what was reflected in the scores as distrust reflecting a more attentive eye to competence and value in the healthcare system. There was no relationship noted with the middle range or lower perceived financial status groups and healthcare system distrust. On the other hand, annual income did not reveal a relationship with healthcare system distrust on any level. Further, socioeconomic status (by both measures of annual income and perceived financial status) was not related to lung cancer stigma, smoking status, or time to seek help in lung cancer.

\section{Ethnicity.}

When ethnicity was examined as a potential confounding variable on the independent variables individually, the only independent variable that was significantly related to ethnicity was lung cancer stigma tested through one-way between-groups analysis of variance $(F(2,90)=3.414, p<.05)$. Specifically, African-Americans had higher levels of lung cancer stigma as indicated by total CLCSS scores than Caucasian individuals in this sample. The mean and standard deviation on the CLCSS was $75.1+12.46$ for African-American participants versus $67.2 \pm 10.93$ for Caucasians. On a measure of lung cancer stigma ranging from 31 (lower level of lung cancer stigma) to 124 (high level of lung cancer stigma), both African-Americans and Caucasians reported modest levels of lung cancer stigma. Higher levels of lung cancer stigma reported by African-Americans compared to Caucasians were an expected finding. As mentioned in earlier discussion, African-Americans may experience more 
generalized stigma related to racial and social discrimination on a daily basis (Lekas et al., 2011) that may be reflected in these findings. However, the mean lung cancer stigma score for African-Americans (75.1) was only 7.9 points higher than Caucasian participants (67.2). Both are considered modest levels of lung cancer stigma suggesting the existence of lung cancer stigma in lung cancer patients regardless of ethnicity.

\section{Social desirability.}

Social desirability is a distorted response on a test item in response to what an individual thinks is a socially acceptable answer (Crowne \& Marlowe, 1960). Lung cancer stigma and healthcare system distrust are sensitive topics and the possibility of socially desirable responses was considered. Social desirability was measured with the 20-item dichotomous Modified MarloweCrowne Social Desirability Scale (Strahan \& Gerbasi, 1972), and was not inversely related to healthcare system distrust, but was inversely related to lung cancer stigma. There was a small, negative weak correlation $(r=-.227, \mathrm{n}=93, p$ $<.05)$ between social desirability and lung cancer stigma. Therefore, study participants that scored higher social desirability scores had lower lung cancer stigma scores. This may have impacted the overall report of lung cancer stigma. The overall mean lung cancer stigma score for this sample was 68.60 supporting a modest level of lung cancer stigma overall in this sample. Perhaps true lung cancer stigma scores in this sample are slightly higher but have been lowered by the influence of social desirable responding. 


\section{Aim 3: Determine the Predictive Power of the Model for Timing in Help- Seeking Behavior in Lung Cancer Patients}

The overall hypothesis was greater healthcare system distrust, higher levels of self-perceived lung cancer stigma, and positive smoking status predict increased time from symptom onset to help-seeking, controlling social desirability, socioeconomic status, and ethnicity. Hierarchical linear regression was used to test this hypothesis. Of the independent variables, the findings revealed that healthcare system distrust and lung cancer stigma were predictive of increased time to seek help in symptoms suggestive of lung cancer. Smoking status was not a statistically significant predictor variable.

Hierarchical regression modeling did support significance in the overall hypothesis. Socioeconomic status, ethnicity, and social desirability were chosen to enter in step one because they were identified as potential confounding variables from a review of the literature (Bibb, 2001; Facione et al., 2002;

Friedman et al., 2006; Henderson et al., 2012; Lowndes et al, 2012). In Step 1 of the hierarchical linear regression model, ethnicity and perceived financial status were identified as unique contributors explaining $10 \%$ of the variance in time to seek help in lung cancer. In Step 2, healthcare system distrust and lung cancer stigma were identified as unique contributors to the overall model adding an additional $13 \%$ explanation of variance in time to seek help in lung cancer for a total variance explained by the model as a whole of $23 \%$. Although lung cancer stigma has not previously been studied as a predictor variable for delayed helpseeking behavior in lung cancer, hierarchical regression modeling supported both 
lung cancer stigma and healthcare system distrust as unique contributors to the final model. The finding of healthcare system distrust as predictive in time to seek help in lung cancer is similar to recent findings in breast cancer screening research in which healthcare system distrust was a significant predictor for screening behavior (Katapodi et al., 2010).

The finding that smoking status was not statistically significant in this sample is important as researchers move forward with future studies examining help-seeking behavior in symptoms suggestive of lung cancer. As previously discussed, smoking status was not correlated to time to seek help in lung cancer symptoms. Given this preliminary statistical analysis after data were collected, it was expected that smoking status would not be a unique contributor to the final model. This may be a reflection of this sample and the equal distribution of never smokers $(32.3 \%)$, former smokers (35.5\%), and current smokers $(32.3 \%)$ at lung cancer diagnosis, or perhaps there were other variables that were more influential in time to seek help, such as lung cancer stigma, that were more pronounced in this study. A positive smoking status has been associated with delayed help-seeking behavior in lung cancer patients (Cataldo et al., 2012; Stuber et al., 2008). This is thought to be related to a masking of symptoms by the sequela of tobacco use or attributing the symptoms to tobacco smoking. The high percentage of former and never smokers $(67.8 \%)$ was likely influential in the non-significance of smoking status on time to seek help in this sample because there were more participants that did not smoke than did at lung cancer diagnosis. 
Of equal importance is the significance of both healthcare system distrust and lung cancer stigma as predictive of greater time to seek help. To date, there have been no studies exploring healthcare system distrust or lung cancer stigma in help-seeking behavior in lung cancer. Although it was plausible that healthcare system distrust and lung cancer stigma would both be important factors in the time to seek help in lung cancer, the findings from this study adds an increased understanding of those variables. Specifically, healthcare system distrust and lung cancer stigma are both predictive of delayed help-seeking behavior in symptoms suggestive of lung cancer.

\section{Recommendations}

Research in the area of help-seeking behavior in lung cancer patients is novel. However, given the current morbidity and mortality rates associated with lung cancer, understanding help-seeking behavior is important and clinically relevant. This study represents an important step in understanding help-seeking behavior in lung cancer patients. This is the first study to examine specific predictor variables in a regression model that influence the timing of help-seeking behavior in lung cancer symptoms and thus delayed help-seeking behavior. Previous studies have examined predictors of delay in acute cardiovascular events (Altice \& Madigan, 2011; Bird et al., 2009; Dracup et al., 2003; Khraim et al., 2009; Zerwic et al., 2007) and breast cancer (Bish et al., 2005; Gould et al., 2010). However, help-seeking behavior in lung cancer is not synonymous with help-seeking behavior in acute cardiovascular events or breast cancer. Lung cancer is a different disease process with a different trajectory including 
presentation, progression, and evaluation. Therefore, it is essential to gain an understanding of help-seeking behavior in the context of lung cancer symptoms. The results from this study provide a beginning conversation about help-seeking behavior in lung cancer. The following sections will discussion recommendations from the findings of this study in regards to theory, practice, policy, research, and instrumentation.

\section{Theory}

Although this study was not a theoretical study, the conceptual model used is the first model to date focused on help-seeking behavior in lung cancer. In a practice discipline such as nursing, conceptual models have value only to the extent that they are applicable to practice. Ultimately, positive health behaviors and outcomes are the goal of nursing. It is important that intervention research in lung cancer be conducted to determine whether or not the timing of help-seeking behavior in individuals with symptoms suggestive of lung cancer can be hastened. A model focused on the key concepts in the phenomenon of help-seeking behavior in the context of lung cancer can help researchers understand important time points. Further, this model can be tested in future studies with lung cancer patients. Key findings can be used to guide the development of interventions focused on hastening help-seeking behavior in lung cancer symptoms by identifying important places in the trajectory that are amenable to change. There were several constructs in this model (Figure 1.2, page 20) that were not tested in this study. Future research should include exploring knowledge of lung cancer, attitudes to help-seeking, disclosure of 
symptoms, and intentions to seek help in lung cancer to more completely understand the phenomenon of delayed help-seeking in lung cancer symptoms.

This study as well as future studies testing the constructs of the conceptual model can be used to guide theory construction. A middle range theory specific to help-seeking behavior in symptoms suggestive of lung cancer can be developed from this model, and can facilitate further understanding of the phenomenon and reveal possible interventions applicable to help-seeking behavior in lung cancer symptoms (Peterson \& Bredow, 2009). A middle range theory would also be helpful in evaluating the effectiveness of interventions.

\section{Practice}

While addressing the issue of decreasing the time to diagnosis in lung cancer is multifactorial, understanding factors that influence the time to seek help in symptoms suggestive of lung cancer is one important aspect that research can address. These findings can have important practice implications. This study supports healthcare system distrust and lung cancer stigma as important variables that impact help-seeking behavior in symptoms suggestive of lung cancer. Healthcare providers, social workers, and organizations promoting lung cancer awareness can benefit from the results of this study. Nurses, in particular, may have the largest impact. Nurses from a variety of specialties (i.e. primary care, family medicine, community health nursing, emergency nursing) can benefit from the results of this study by an increased awareness that many lung cancer patients experience healthcare system distrust and lung cancer stigma. To date, these variables are not discussed in the literature in the context of delayed help- 
seeking behavior. These variables can serve as potential barriers to a timely diagnosis.

Nurses may be the first healthcare professional that an individual with lung cancer has contact with before they are diagnosed if they enter the healthcare system for other reasons. Nurses have an opportunity to assess for symptoms that the individual may not recognize as concerning. The findings from this study suggest that lung cancer stigma and healthcare system distrust can serve as barriers to seeking help for lung cancer symptoms. Knowledge of the existence of these variables can better inform frontline healthcare providers in their interactions with patients. Efforts to combat the perception of healthcare system distrust and lung cancer stigma starts with healthcare providers. Nurses are on the frontlines of public health issues. Healthcare system distrust and lung cancer stigma are public health issues that are barriers to timely help-seeking behavior. The findings from this study support the need for a concerted public health awareness effort targeting lung cancer-related stigma and healthcare system distrust.

Lung cancer stigma has historically grown out of the misconception that lung cancer is a self-inflicted disease and a disease exclusive to smokers. Public health awareness regarding other risk factors (such as radon, secondhand smoke and other environmental exposure, and genetic susceptibility) as well as the fact that non-smokers develop lung cancer is an important piece in efforts to decrease the stigma associated with lung cancer. Future intervention work 
should focus on decreasing the stigma associated with lung cancer as well as increasing trust in the healthcare system.

Nurses have repeatedly ranked at the top of the list in the honesty and ethics in professions annual Gallup poll (Gallup poll, 2012). Hence, nurses are in a unique position to address healthcare system distrust. This is not something that can be addressed alone. Future research should expand on understanding this phenomenon more completely in lung cancer. Recent research has explored racial discrimination and healthcare system distrust (Armstrong et al., 2013) documenting a higher level of healthcare system distrust in African-Americans. The findings of this study revealed a similar level of healthcare system distrust in African-Americans and Caucasians suggesting healthcare system distrust may be more closely associated with the disease process versus ethnicity in lung cancer. Knowledge of healthcare system distrust, regardless of ethnicity, in lung cancer is important for nurses to understand so the target population of their efforts is not confined to one ethnic background but rather lung cancer patients in general.

This study illuminates meaningful factors that are related to help-seeking behavior in lung cancer that can be used to focus patient and provider educational messages. It is important that educational messages concerning lung cancer include a component that addresses healthcare system distrust and lung cancer stigma. It is also important that these messages target the appropriate audiences: (a) the public at large, (b) those at high risk for lung cancer development and, (c) those at high risk for propensity to delay seeking 
help for lung cancer symptoms. Educational messages should target increasing public awareness about lung cancer, risk factors, staging and implications of early versus late stage diagnosis. While current awareness is focused on a target population of smokers, equally important is public awareness of the existence of lung cancer in never smokers and former smokers. Educational messages should focus on increasing the awareness of lung cancer in never smokers and former smokers in efforts to de-stigmatize the disease process. Although tobacco smoking increases the risk of lung cancer development, the public needs to be better informed that other non-smoking related factors are associated with lung cancer.

For individuals at high risk of developing lung cancer, lung cancer screening programs need to target these high-risk populations in efforts of detecting lung cancer early. A component of an effective lung cancer screening program is education. Patient education regarding signs and symptoms of lung cancer (such as recurrent cough or respiratory change, hemoptysis, pain, fatigue, or weight loss) are important intervention components for public education. Just as public awareness of common signs and symptoms of acute cardiovascular events was critical decades ago (Bandura, 2004; Green \& Kreuter, 1990), there is a current need to educate the public about lung cancer. Studies point to the longest period of delay in the time span from the first symptom an individual becomes aware of to the time of seeking help for that symptom in the diagnosis of lung cancer (Corner et al., 2005; Tod \& Joanne, 2010). This is the time period 
that public awareness and patient education must target. This is a key component in the process of decreasing the time to diagnosis in lung cancer.

Key sociodemographic variables were also found to be important in delayed help-seeking behavior in lung cancer patients. First, findings from this study indicated gender differences with lung cancer stigma. Awareness of the disproportionate perception of lung cancer stigma among females versus males is an important component of understanding help-seeking behavior in lung cancer particularly since lung cancer affects both genders equally. Provider awareness of the propensity of women to experience lung cancer stigma more often than men can help providers focus their efforts to decrease perceived stigma in this group by dispelling common myths associated with lung cancer. A related issue to lung cancer stigma involves social desirability. Social desirability can be a difficult phenomenon to tackle in practice. However, it is noteworthy in the context of lung cancer, and healthcare providers should be aware of its existence when interacting with individuals at high risk for and those with diagnosed lung cancer. Care and thought should be given to interactions in efforts to promote a positive and nonjudgmental patient-provider relationship.

Finally, although not included as an independent variable in this study, the findings from the regression model supported the importance of ethnicity as a unique contributor to the predictive potential of delayed help-seeking behavior. Ethnicity was entered into the hierarchical regression model as a confounding variable and was found to be a unique contributor to the model. Cultural competence in provider interactions with lung cancer patients and in public health 
awareness campaigns about lung cancer is critical to their success. Furthermore, specific to lung cancer diagnosis and treatment, an important goal for healthcare providers is to provide culturally concordant care. To be successful in fostering early help-seeking behavior in lung cancer symptoms as well as adherence to treatment regimens and compliance with follow-up plans after diagnosis depends on clinical care that respects an individual's cultural background.

\section{Policy}

Findings from this study lead to several policy related recommendations. This study supports an overarching assertion that increased public health awareness about lung cancer is paramount. Specifically, public health awareness in lung cancer should target increasing awareness of lung cancer symptoms, decreasing lung cancer related stigma, the significant proportion of lung cancer patients that have never smoked, and decreasing delays in help-seeking behavior in symptoms suggestive of lung cancer. Health policy can address all components by allocating federal level funding to intervention research and public health awareness campaigns.

First, research to develop effective interventions that target increasing awareness of lung cancer symptoms, destigmatizating lung cancer and its public perception, and promoting timely help-seeking behavior in lung cancer symptoms is needed. Second, lung cancer is a public health crisis. As the deadliest cancer worldwide (ACS, 2013), lung cancer remains poorly understood in the public arena. For example, most individuals think that people with lung cancer are smokers. This is not true. While smoking is acknowledged as the number one 
risk factor for the development of lung cancer (ACS, 2013), the significant proportion of lung cancer patients that have never smoked in this study point to a problem that goes beyond tobacco smoking. In fact, radon is the second leading risk factor for lung cancer (ACS, 2013) yet most people are unaware of this nonsmoking related risk factor.

Healthcare providers, healthcare researchers, and the public do not fully understand lung cancer in never smokers. Therefore, national health policy language (such as language of the Lung Cancer Mortality Reduction Act) should be expanded to include non-smoking related risk factors to widen the parameters of lung cancer screening. For example, it is documented that prolonged radon exposure and secondhand smoke exposure increase the risk for lung cancer (ACS, 2013). These individuals should be included in the American Cancer Society's (2013) lung cancer screening guidelines. Funding for research that focuses on understanding risk factors for lung cancer in never smokers will validate the inclusion of appropriate risk factors in this population. Expansion of the lung cancer screening guideline parameters will help capture never smokers that may be at an increased risk for lung cancer that would otherwise not be detected.

\section{Research}

This study examined help-seeking behavior from the perspective of the individual in the context of a cancer that is associated with a low survival rate. Delay from the perspective of the individual (as opposed to provider or healthcare system) has been identified as the longest time span of delay in lung 
cancer (Corner et al., 2005). Help-seeking behavior from the perspective of the individual is an important piece as research moves forward to address in decreasing the time to diagnosis. Future studies should focus on building a program of research that explores the multiple venues of delay that impact the time to diagnosis in lung cancer to address the disparate survival rates. The following section will describe several research related recommendations.

With the identification of healthcare system distrust and lung cancer stigma as unique predictors of delayed help-seeking behavior in lung cancer symptoms, future research should examine these predictors more closely. In addition, other potential predictor variables should be investigated to increase the understanding of the phenomenon of delayed help-seeking behavior in this disease. For example, ethnicity was noted as a unique contributor to the predictive potential of help-seeking behavior in lung cancer symptoms. Future intervention research with help-seeking behavior in lung cancer must remain cognizant of the potential influence of ethnicity when designing patient educational programs and public health awareness campaigns. Understanding other potential predictor variables, such as ethnicity, more fully will guide future intervention research.

Another variable, smoking status, was not found to be significant in any of the statistical analyses of this study. Perhaps this provides an important glimpse into the dynamics of individuals with lung cancer. Although lung cancer is strongly associated with tobacco smoking, perhaps smoking status does not play as large a part as previously thought in time to seek help in lung cancer. This is 
not to suggest that smoking status should not be used to assess risk for lung cancer, but rather the importance of not recognizing that nonsmokers are at risk for lung cancer. Numerous risk factors for lung cancer have been identified including exposure to secondhand smoke, radon and other chemicals as well as a history of tuberculosis (ACS, 2013). Future research must focus on effective screening mechanisms for never smokers to identify them earlier in the trajectory of their lung cancer diagnosis.

Although it has been reported that only 10 to $15 \%$ of lung cancer patients are never smokers (Thun et al., 2006), this study consisted of $32.3 \%$ never smokers. A previous pilot study with lung cancer patients conducted by the researcher had a sample that consisted of $36.4 \%$ never smokers. This challenges previous findings and underscores the significance of lung cancer patients that have never smoked. Perhaps this is representative of the geographic area, or perhaps previous reports of lung cancer patients that never smoked are no longer accurate. Future research should examine this population more closely as well as explore the potential differences in never smokers and former/current smokers. Research should also explore help-seeking behavior in lung cancer patients that have never smoked comparatively with lung cancer patients that have a smoking history to identify potential differences. Findings can guide the development of effective interventions that target the appropriate population.

This study also revealed the importance of socioeconomic status as measured by perceived financial status as relevant in the larger picture of time to 
seek help in lung cancer. Although there are several ways to measure socioeconomic status, this study supports the importance of perceived financial status as an important factor to consider. This study confirmed that arbitrary delimitations found in annual income brackets are not correlated with time to seek help reinforcing the notion that socioeconomic status is a multidimensional construct that is complex and deserves a more focused investigation. To understand the full impact of socioeconomic status on help-seeking behavior can help identify the target population for future intervention work.

Key information was also gleaned in this study regarding the sociodemographic group most associated with healthcare system distrust. While the higher perceived financial status group unexpectedly scored higher on healthcare system distrust, this raises important questions regarding distrust of the healthcare system and its etiology. This is not information that can be easily assessed quantitatively. Future research should follow up on this finding with a qualitative component to better understand healthcare system distrust in higher socioeconomic individuals.

\section{Instrumentation}

Findings from this study revealed several recommendations related to instrumentation. The following discussion will address recommendations regarding the Revised Healthcare System Distrust Scale (RHCSDS), Cataldo Lung Cancer Stigma Scale (CLCSS), and the investigator-developed Timing of Help-Seeking Behavior and Demographic Questionnaire (THSBDQ). 
Distrust implies the loss of trust or loss of a relationship. The RHCSDS measures two dimensions: values congruence and technical competence. Trust in the healthcare system may be greater than the sum of values and competence. Perceived technical incompetence can certainly impact the level of trust, but does not necessarily equate to healthcare system distrust. Equally, values incongruence may be an aspect of healthcare system distrust but does not fully capture the phenomenon. Future research should involve psychometric studies to increase the multidimensional measure of the RHCSDS from its current dual dimensional measure.

The CLCSS is a 31-item Likert-type scale that had strong internal consistency reliability in this study (Cronbach's coefficient alpha of .95) and in the original psychometric study (Cronbach's coefficient alpha of .96, Cataldo et al., 2011). However, practical application of the instrument revealed a level of redundancy that interfered with its administration. Participants frequently questioned the investigator about the repetitive nature of some questions during administration of the instrument. The high Cronbach's coefficient alpha may indicate there is some level of redundancy in the four subscales. Future research should include a development of a shortened composite measure of the CLCSS and the validation of the shortened CLCSS. A shortened measure could lessen participant burden in future studies that use this scale.

The THSBDQ was a useful instrument to gather information regarding the timing of help-seeking behavior in symptoms suggestive of lung cancer. However, in hindsight, there were several additions that would have provided a 
more complete picture of help-seeking behavior. Future uses of this instrument should add the following items: (a) family history of lung cancer, (b) exposure to secondhand smoke and if positive, length of time exposed, (c) zip code data, (d) geographic location, (e) access to transportation, (f) access to healthcare, (g) primary care provider access, and (h) number of family members in the household. Adding these items would make the THSBDQ more inclusive of important sociodemographic variables that may influence the time to seek help in lung cancer symptoms.

\section{Limitations and Strengths}

This study represents an important component of understanding delayed help-seeking behavior in symptoms suggestive of lung cancer and the study has many strengths. However, this study is not without limitations. It is important that these limitations be considered. The following will discuss the limitations and strengths of the study.

First, the age of the average participant was almost ten years younger than the majority of individuals diagnosed with lung cancer. Younger individuals may perceive their symptoms differently than their older counterparts and this may affect recognition and behavioral response to their symptoms. In addition, the study sample was nearly two-thirds women and this is higher than the national average of lung cancer patients (ACS, 2013). Help-seeking behavior in lung cancer may be different for women compared to men. Future studies should try to emulate the national population of lung cancer patients more closely. However, although a younger sample and higher proportion of female 
participants may be viewed as a limitation, it is also a strength of the study. The inclusion of younger individuals with lung cancer provided the opportunity to statistically analyze the variable of age which had no statistical significance on the timing of help-seeking behavior in lung cancer symptoms. In addition, the female perspective of help-seeking behavior in lung cancer is important in practice, research and theory.

Another potential limitation is zip code data were not collected. Zip code data could have allowed examination of potential differences in rural versus nonrural/urban versus non-urban individuals. With a greater percentage of study participants recruited from an academic cancer center $(77.4 \%)$ that treats patients from a wide geographic area encompassing both Indiana and Kentucky, examination of these differences may have provided richer details. These details could have provided a better understanding of the sample in relation to healthcare system distrust and lung cancer stigma.

Alternatively, there are several strengths in the demographic composition of the study sample worth noting. First, the percentage of African-Americans that participated in this study (17.2\%) was strong and representative of the geographic region. This is important when generalizing to the population. Although the African-American sample was predominantly female, the number of African-Americans recruited to participate was suitable. African-Americans have been historically underrepresented in healthcare research (Corbie-Smith et al., 2002; Corbie-Smith et al., 1999; Gamble, 1997; Moseley et al., 2007). This study provides an African-American perspective to help-seeking behavior in lung 
cancer symptoms. Another demographic variable worth highlighting is the percentage of never smokers (32.3\%). As mentioned previously, the percentage of never smokers with lung cancer has been reported at lower numbers than in this sample. However, the fact that never smokers were present in this study in significant numbers revealed an important target population for future studies in lung cancer. Finally, this study included lung cancer patients diagnosed with both early and late stage lung cancer. This provided a more complete picture of helpseeking behavior in lung cancer patients.

Concerning the demographic questionnaire, as mentioned in the previous recommendation section, there are several items that should be included in future studies to provide a more robust and complete picture of help-seeking behavior in lung cancer patients. For example, the inclusion of questions regarding family history of lung cancer, exposure to secondhand smoke, primary care provider access, geographic location, transportation, and number of family members in the household would have provided additional key information. Data reflecting the total number of household members would have added strength to understanding the socioeconomic status of the participants. Family history of lung cancer could be viewed as an influential sociodemographic variable and thus a potential predictor of seeking help in lung cancer symptoms. Specifically, family history of lung cancer could influence the recognition of symptoms as lung cancer as well as the timing of help-seeking behavior.

Another risk factor for lung cancer that could have been assessed is exposure to secondhand smoke (ACS, 2013). This is an identified risk factor for 
lung cancer, and examining this would have provided a more complete assessment of risk factors for lung cancer. However, this study involved assessing variables that may influence the time to seek help in lung cancer. While secondhand smoke exposure is a risk factor for the development of lung cancer, it may not have a direct impact on the time to seek help in lung cancer.

A key limitation to the study was recall bias. Data related to symptom awareness and the timing of help-seeking behavior was collected retrospectively. In the design of the study, it was anticipated that individuals may not accurately recall specific symptoms and dates. This inherent limitation was addressed with key event mapping (Molassiotis et al., 2010) in which the researcher used a calendar to assist the participant in recall by asking the participant to remember in relation to key personal events and common key events on a calendar. In data collection, many participants did not have difficulty recalling symptoms or timing. However, when participants did have difficulty with recall, this method was employed and was successful.

A strength of the study design was the in-person data collection procedure which provided a richer understanding of the perception of lung cancer symptoms and the timing of help-seeking behavior for the researcher. This allowed the researcher to develop a rapport with the participant during data collection which allowed the participant to share anecdotal stories related to their lung cancer diagnosis. It also allowed the researcher to ensure that all survey items were addressed resulting in a lack of missing data in this sample. Lack of missing data provided a full data set for statistical analysis. Another strength was 
the use of survey methodology which allowed for measuring many sample characteristics of lung cancer patients. While this was not the central focus of this study, it provided key information that highlighted the significant number of never smokers in the sample.

Finally, a limitation to the design of this study was the lack of assessing the individual's perception of symptom seriousness. This could influence the time to seek help in symptoms suggestive of lung cancer. While the purpose of the study was focused on the independent variables and their relationship to the dependent variable, the perception of symptom seriousness is important. To more fully understand the symptoms that are perceived in a study focused on help-seeking behavior in lung cancer, perception of symptom seriousness should be included as a measure.

\section{Conclusion}

The purpose of this study was to examine the influence of healthcare system distrust, lung cancer stigma, and smoking status on the timing of helpseeking behavior in symptoms suggestive of lung cancer. Healthcare system distrust and lung cancer stigma were found influential and predictive of greater time to seek help in lung cancer. The discussion addresses the study findings overall, including expected and unexpected findings, and possible explanations for significant and non-significant findings. The study results have significant implications for nursing practice, healthcare research, health policy, and theory. To a greater extent, the study results have significant public health implications, particularly in relation to patient and public education. Implications for theory, 
practice, policy, research, and instrumentation are also addressed. It is important that this area of inquiry be investigated further. 


\section{REFERENCES}

Aberle, D. R., Adams, A. M., Berg, C. D., Black, W. C., Clapp, J. D., Fagerstrom, R. M.,...Sicks, J. D. (2011). Reduced lung-cancer mortality with low-dose computed tomographic screening. The New England Journal of Medicine, 365(5), 395-409. doi:10.1056/NEJMoa1102873

Adler, N. E. (1994). Socioeconomic status and health: The challenge of the gradient. American Psychologist, 49(1), 15.

Ajzen, I. (1991). The theory of planned behavior. Organizational Behavior and Human Decision Processes, 50, 179-211.

Altice, N. F., \& Madigan, E. A. (2011). Factors associated with delayed careseeking in hospitalized patients with heart failure. Heart \& Lung, 1-11. doi:10.1016/j.hrtlng.2011.09.007

American Cancer Society. Cancer Facts \& Figures 2013. Atlanta, GA: American Cancer Society, 2013.

Andersen, R. S., Vedsted, P., Olesen, F., Bro, F., \& Sondergaard, J. (2011).

Does the organizational structure of health care systems influence careseeking decisions? A qualitative analysis of Danish cancer patients' reflections on care-seeking. Scandinavian Journal of Primary Health Care, 29(3), 144-149.

Armstrong, K., McMurphy, S., Dean, L. T., Micco, E., Putt, M., Halbert, C. H.,...Shea, J. A. (2008). Differences in the patterns of health care system 
distrust between blacks and whites. Journal of General Internal Medicine, 23(6), 827-833. doi:10.1007/s11606-008-0561-9

Armstrong, K., Putt, M., Halbert, C. H., Grande, D., Schwartz, J. S., Liao, K.,...Shea, J. A. (2013). Prior experiences of racial discrimination and racial differences in health care system distrust. Medical Care, 51(2), 144-150. doi:10.1097/MLR.0b013e31827310a1

Armstrong, K., Putt, M., Halbert, C. H., Grande, D., Schwartz, J. S., Liao, K.,...Shea, J. (2012). The influence of health care policies and health care system distrust on willingness to undergo genetic testing. Medical Care, 50(5), 381-387. doi:10.1097/MLR.0b013e31824d748b

Armstrong, K., Ravenell, K. L., McMurphy, S., \& Putt, M. (2007). Racial/ethnic differences in physician distrust in the United States. American Journal of Public Health, 97(7), 1283-1289. doi:10.2105/AJPH.2005.080762

Ashton, K. C. (1999). How men and women with heart disease seek care: The delay experience. Progress in Cardiovascular Nursing, 14(2), 53-74.

Bandura, A. (2004). Health promotion by social cognitive means. Health Education Behavior, 31(2), 143-164. doi:10.1177/1090198104263660

Banks, A. D., \& Dracup, K. (2006). Factors associated with prolonged prehospital delay of African Americans with acute myocardial infarction. American Journal of Critical Care, 15(2), 149-157.

Baumann, T. (2004). Defining ethnicity. The SAA Archaeological Record, 12-14.

Bell, K., Salmon, A., Bowers, M., Bell, J., \& McCullough, L. (2010). Smoking, stigma and tobacco 'denormalization': Further reflections on the use of 
stigma as a public health tool. A commentary on Social Science \& Medicine's stigma, prejudice, discrimination and health special issue (67:3), Social Science \& Medicine, 70, 795-799.

doi:10.1016/j_socscimed.2009.09.060

Berger, B., Ferrans, C., \& Lashley, F. (2001). Measuring stigma in people with HIV: Psychometric assessment of the HIV stigma scale. Research in Nursing and Health, 24, 518-529.

Berkanovic, E., Telesky, C., \& Reeder, S. (1981). Structural and social psychological factors in the decision to seek medical care for symptoms. Medical Care, 19(7), 693-709.

Bibb, S. C. (2001). The relationship between access and stage at diagnosis of breast cancer in African American and Caucasian women. Oncology Nursing Forum, 28(4), 711-719.

Bird, M. W., Woods, A. G., \& Warren, N. A. (2009). Factors influencing treatment delays for acute myocardial infarction. Critical Care Nursing Quarterly, 32(1), 19-23.

Bish, A., Ramirez, A., Burgess, C., \& Hunter, M. (2005). Understanding why women delay in seeking help for breast cancer symptoms. Journal of Psychosomatic Research, 58, 321-326.

doi:10.1016/j.psychores.2004.10.007

Blair, A., \& Freeman, L. B. (2006). Lung cancer among nonsmokers. Epidemiology, 17(6), 601-603.

Brink, E., Karlson, B. W., \& Hallberg, L. R. M. (2002). To be stricken with acute 
myocardial infarction: A grounded theory study of symptom perception and care-seeking behavior. Journal of Health Psychology, 7(5), 533-543. doi:10.1177/1359105302007005673

Brown, J. K., Cooley, M. E., Chernecky, C., \& Sarna, L. (2011). A symptom cluster and sentinel symptom experience by women with lung cancer. Oncology Nursing Forum, 38(6), E425-435. doi:10.1188/11.ONF.E425-E435

Burgess, C. C., Bish, A. M., Hunter, H. S., Salkovskis, P., Michell, M., Whelehan, P., \& Ramirez, A. J. (2008). Promoting early presentation of breast cancer: Development of a psycho-educational intervention. Chronic IIIness, 4, 13-27. doi:10.1177/1742395307084404

Burgess, C. C., Linsell, L., Kapari, M., Omar, L., Michell, M., Whelehan, P.,... Ramirez, A. J. (2009). Promoting early presentation of breast cancer by older women: A preliminary evaluation of a one-to-one health professional-delivered intervention. Journal of Psychosomatic Research, 67, 377-387. doi:10.1177/1742395307084404

Burgess, C. C., Ramirez, A. J., Richards, M. A., \& Love, S. B. (1998). Who and what influences delayed presentation in breast cancer? British Journal of Cancer, 77(8), 1343-1348.

Burmeister, B. H., Zarate, D. D., Burmeister, E. A., Harden, H. E., Colquist, S. P., Cossio, D. L.,...Walpole, E. T. (2010). Lung cancer patients in Queensland suffer delays in receiving radiation therapy - but not as a result of distance. Internal Medicine Journal, 40, 126-132. 
doi:10.1111/j.1445-5994.2009.01912.x

Burnett, R. E., Blumenthal, J. A., Mark, D. B., Leimberger, J. D., \& Califf, R. M. (1995). Distinguishing between early and late responders to symptoms of acute myocardial infarction. The American Journal of Cardiology, 75, 1019-1022.

Caplan, L. S. (1995). Patient delay in seeking help for potential breast cancer. Public Health Reviews, 23(3), 263-274.

Caplan, L. S., \& Helzlsouer, K. J. (1992). Delay in breast cancer: A review of the literature. Public Health Review, 20(3-4), 187-214.

Caplan, L. S., Helzlsouer, K. J., Shapiro, S., Wesley, M. N., \& Edwards, B. K. (1996). Reasons for delay in breast cancer diagnosis. Preventive Medicine, 25, 218-224.

Carney, R., Fitzsimons, D., \& Dempster, M. (2002). Why people experiencing myocardial infarction delay seeking medical assistance. European Journal of Cardiovascular Nursing, 1, 237-242.

Cataldo, J. K., Jahan, T. M., \& Pongquan, V. L. (2012). Lung cancer stigma, depression, and quality of life among ever and never smokers. European Journal of Oncology Nursing, 16, 264-269. doi:10.1016/j.ejon.2011.06.008

Cataldo, J. K., Slaughter, R., Jahan, T. M., Pongquan, V., \& Hwang, W. J. (2011). Measuring stigma in people with lung cancer: Psychometric testing of the Cataldo Lung Cancer Stigma Scale. Oncology Nursing Forum, 38(1), E46-E54.

Centers for Disease Control and Prevention, Social Determinants of Health- 
Definitions (2011), Retrieved from

http://www.cdc.gov/socialdeterminants/Definitions.html

Centers for Disease Control and Prevention, Tobacco control state highlights 2012, Retrieved from http://www.cdc.gov/tobacco/data_statistics/state_data/index.htm Centers for Disease Control and Prevention, State-specific secondhand smoke exposure and current cigarette smoking among adults: United States, 2008. MMWR Morbidity and Mortality Weekly Report 2009, 58, 12321235.

Chapple, A., Ziebland, S., \& McPherson, A. (2004). Stigma, shame, and blame experienced by patients with lung cancer: A qualitative study. British Medical Journal, 328(7454), 1-5. doi:10.1136/bmj.38111.639734.7C

Chen, E., Nguyen, J., Cramarossa, G., Khan, L., Leung, A., Lutz, S., \& Chow, E. (2011). Symptom clusters in patients with lung cancer: A literature review. Expert Reviews of Pharmacoeconomics \& Outcomes Research, 11(4), 433-439. doi:10.1586/ERP.11.56

Christensen, E. D., Harvald, T., Jendresen, M., Aggestrup, S., \& Petterson, G. (1997). The impact of delayed diagnosis of lung cancer on the stage at the time of operation. European Journal of Cardio-thoracic Surgery, 12, 880884.

Cohen, J. (1992). Quantitative methods in psychology: A power primer. Psychological Bulletin, 112(1), 155-159.

Coates, R. J., Bransfield, D. D., Wesley, M., Hankey, B., Eley, W., Greenberg, 
R. S., Flanders, D., Hunter, C. P., Edwards, B. K., Forman, M., Chen, V. W., Reynolds, P., Boyd, P., Austin, D., Muss, H., Blacklow, R. S. (1992). Black/white survival study group: Differences between black and white women with breast cancer in time from symptom recognition to medical consultation. Journal of the National Cancer Institute, 84(12), 938-950.

Corbie-Smith, G., Thomas, S. B., \& St. George, D. M. M. (2002). Distrust, race, and research. Archives of Internal Medicine, 162, 2458-2463.

Corbie-Smith, G., Thomas, S. B., Williams, M., \& Moody-Ayers, S. (1999). Attitudes and beliefs of African Americans toward participation in medical research. Journal of General Internal Medicine, 14, 537-546.

Cornally, N., \& McCarthy, G. (2011). Help-seeking behaviour: A concept analysis. International Journal of Nursing Practice, 17(3), 280-288. doi:10.1111/j.1440-172X.2011.01936.x

Corner, J., Hopkinson, J., Fitzsimmons, D., Barclay, S., \& Muers, M. (2005). Is late diagnosis of lung cancer inevitable? Interview study of patients' recollections of symptoms before diagnosis. Thorax, 60, 314-319. doi:10.1136/thx.2004.029264

Corner, J., Hopkinson, J., \& Roffe, L. (2006). Experience of health changes and reasons for delay in seeking care: A UK study of the months prior to the diagnosis of lung cancer. Social Science \& Medicine, 62, 1381-1391. doi:10.1016/j.socscimed.2005.08.012 Crocker, J., Major, B., \& Steele, C. (1998). Social stigma. In D. T. Gilbert, S. T. 
Fiske, \& G. Lindzey (Eds.), The handbook of social psychology (pp. 504553). New York, NY: McGraw-Hill.

Crowne, D. P., \& Marlowe, D. (1960). A new scale of social desirability independent of psychopathology. Journal of Consulting Psychology, 24, 349-354.

Cunningham, C. O., Sohler, N. L., Korin, L., Gao, W., \& Anastos, K. (2007). HIV status, trust in health care providers, and distrust in the health care system among Bronx women. AIDS Care, 19(2), 226-234.

doi:10.1080/09540120600774263

De Nooijer, J., Lechner, L., \& De Vries, H. (2002). Early detection of cancer: Knowledge and behavior among Dutch adults. Cancer Detection and Prevention, 26, 362-369.

De Nooijer, J., Lechner, L., \& De Vries, H. (2003). Social psychological correlates of paying attention to cancer symptoms and seeking medical help. Social Science \& Medicine, 56, 915-920.

Department of Defense. (2010). Lung Cancer, Lung Cancer Research Program, Congressionally Directed Medical Research Programs (CDMRP). Retrieved from http://cdmrp.army.mil//crp/default.shtml

Dhamija, S., Sehgal, A., Luthra, U. K., \& Sehgal, K. (1993). Factors associated with awareness and knowledge of cervical cancer in a community: Implication for health education programmes in developing countries. Journal of the Royal Society of the Promotion of Health, 113(4), 184-186.

Dovidio, J. F., Penner, L. A., Albrecht, T. L., Norton, W. E., Gaertner, S. L., \& 
Shelton, J. N. (2008). Disparities and distrust: The implications of psychological processes for understanding racial disparities in health and health care. Social Science \& Medicine, 67(3), 478-486.

doi:10.1016/j.socscimed.2008.03.019

Dracup, K., \& Moser, D. K. (1991). Treatment-seeking behavior among those with signs and symptoms of acute myocardial infarction. Heart \& Lung, 20, $570-575$.

Dracup, K., Moser, D. K., Eisenberg, M., Meischke, H., Alonzo, A. A., \& Braslow, A. (1995). Causes of delay in seeking treatment for heart attack symptoms. Social Science \& Medicine, 40(3), 379-392.

Dracup, K., Moser, D. K., McKinley, S., Ball, C., Yamasaki, K., Kim, C.,...Caldwell, M. A. (2003). An international perspective on the time to treatment for acute myocardial infarction. Journal of Nursing Scholarship, 35(4), 317-323.

Dubayova, T., van Dijk, J. P., Nagyova, I., Rosenberger, J., Havlikova, E., Gdovinova, Z., . . Groothoff. J. W. (2010). The impact of the intensity of fear on patient's delay regarding health care seeking behavior: $A$ systematic review. International Journal of Public Health, 55, 459-468. doi:10.1007/s00038-010-0149-0

Egede, L. E., \& Ellis, C. (2008). Development and testing of the Multidimensional Trust in Health Care Systems Scale. Journal of General Internal Medicine, 23(6), 808-815. doi:10.1007/s11606-008-0613-1

Else-Quest, N. M., LoConte, N. K., Schiller, J. H., \& Hyde, J. S. (2009). 
Perceived stigma, self-blame, and adjustment among lung, breast and prostate cancer patients. Psychology and Health, 24(8), 949-964. doi:10.1080/08870440802074664

Facione, N. C. (1993). Delay versus help seeking for breast cancer symptoms: A critical review of the literature on patient and provider delay. Social Science \& Medicine, 36(12), 1521-1534.

Facione, N. C. (2002). The J-Delay scale: A measure of the likelihood of patient delay in breast cancer. Research and Theory for Nursing Practice: An International Journal, 16(2), 103-118.

Facione, N. C., Dodd, M. J., Holzemer, W., \& Meleis, A. I. (1997). Helpseeking for self-discovered breast symptoms: Implications for early detection. Cancer Practice, 5(4), 220-227.

Facione, N. C., \& Facione, P. A. (2006). The cognitive structuring of patient delay in breast cancer. Social Science \& Medicine, 63, 3137-3149. doi:10.1016/j.socscimed.2006.08.014

Facione, N. C., \& Giancarlo, C. A. (1998). Narratives of breast symptom discovery and cancer diagnosis: Psychologic risk for advanced breast cancer at diagnosis. Cancer Nursing, 21, 430-440.

Facione, N. C., Miaskowski, C., Dodd, M. J., \& Paul, S. M. (2002). The selfreported likelihood of patient delay in breast cancer: New thoughts for early detection. Preventive Medicine, 34, 397-407. doi:10.1006/pmed.2001.0998

Faul, F., Erdfelder, E., Lang, A., \& Buchner, A. (2009). G*Power (Version 3.1.4) 
http://www.psycho.uni-duesseldorf.de/abteilungen/aap/gpower3/

Ferlay, J., Shin, H. R., Bray, F., Forman, D., Mathers, C., \& Parkin, D. M.

GLOBOCAN 2008 v1.2, Cancer incidence and mortality worldwide: IARC CancerBase No. 10. Lyon, France: International Agency for Research on Cancer; 2010.

Available from http://globocan.iarc.fr, accessed on March 12, 2012.

Fox-Wasylyshyn, S. M., El-Masri, M., \& Artinian, N. T. (2010). Testing a model of delayed care-seeking for acute myocardial infarction. Clinical Nursing Research, 19(1), 38-54. doi:10.1177/1054773809353163

Frayne, S. M., Crawford, S. L., McGraw, S. A., Smith, K. W., \& McKinlay, J. B. (2002). Help-seeking behaviors of blacks and whites dying from coronary heart disease. Ethnicity \& Health, 7(2), 77-86.

doi:10.1080/1355785022000038560

Friedman, L. C., Kalidas, M., Elledge, R., Dulay, M. F., Romero, C., Chang, J.,...Liscum, K. R. (2006). Medical and psychosocial predictors of delay in seeking medical consultation for breast symptoms in women in a public sector setting. Journal of Behavioral Medicine, 29(4), 327-334.

doi:10.1007/s10865-006-9059-2

Fukuoka, Y., Dracup, K., Moser, D. K., McKinley, S., Ball, C., Yamasaki, K.,...Kim, C. (2007). Is severity of chest pain a cue for women and men to recognize acute myocardial infarction symptoms as cardiac in origin? Progress in Cardiovascular Nursing, 22,132-137.

Fukuoka, Y., Takeshima, M., Ishii, N., Chikako, M., Makaya, M., Groah, 
L.,...Dracup, K. (2010). An initial analysis: Working hours and delay in seeking care during acute coronary events. American Journal of Emergency Medicine, 28(6), 734-740.

doi:10.1016/j.ajem.2009.06.020

Gallup Poll. (2012). Honesty/Ethics in Professions Report. Retrieved from http://www.gallup.com/poll/1654/honesty-ethics-professions.aspx

Gamble, V. N. (1997). Under the shadow of Tuskegee: African Americans and health care. American Journal of Public Health, 87(11), 1773-1778.

Gibler, W. B., Armstrong, P. W., Ohman, E. M., Weaver, W. D., Stebbins, A. L., Gore, J. M., . . Topol, E. J. (2002). Persistence of delays in presentation and treatment for patients with acute myocardial infarction: the GUSTO I and GUSTO-III experience. Annals of Emergency Medicine, 39(2), 123130.

Gift, A. G., Stommel, M., Jablonski, A., \& Given, W. (2003). A cluster of symptoms over time in patients with lung cancer. Nursing Research, 52(6), 393-400.

Goffman, E. (1963). Stigma: Notes on the management of spoiled identity. New York, NY: Simon \& Schuster.

Gollwitzer, P. M. (1993). Goal achievement: The role of intentions. European Review of Social Psychology, 4, 141-185.

Gould, J., Fitzgerald, B., Fergus, K., Clemons, M., \& Baig, F. (2010). Why women delay seeking assistance for locally advanced breast cancer. Canadian Oncology Nursing Journal, 20(1), 23-29. 
Green, L. W., \& Kreuter, M. W. (1990). Health promotion as a public health strategy for the 1990s. Annual Review of Public Health, 11, 319-334. doi:10.1146/annurev.pu.11.050190.001535

Greene, K., \& Banarjee, S. C. (2008). Disease-related stigma: Comparing predictors of AIDS and cancer stigma. Journal of Homosexuality, 50(4), 185-209. doi:10.1300/J082v50n04_08

Griffith, D. M., Allen, J. O., \& Gunter, K. (2011). Social and cultural factors influence African American men's medical help seeking. Research on Social Work Practice,21(3), 337-347. doi:10.1177/1049731510388669

Gullatte, M. (2006). The influence of spirituality and religiosity on breast cancer screening delay in African American women: Application of the theory of reasoned action and planned behavior (TRA/TPB). The Association of Black Nursing Faculty Journal, 89-94.

Gullatte, M. M., Hardin, P., Kinney, A., Powe, B., \& Mooney, K. (2009). Religious beliefs and delay in breast cancer diagnosis for self-detected breast changes in African-American women. The Journal of the National Black Nurses Association, 20(1), 25-35.

Gullatte, M. M., Phillips, J. M., \& Gibson, L. M. (2006). Factors associated with delays in screening of self-detected breast changes in African-American women. The Journal of National Black Nurses Association, 17(1), 45-50. Harandy, T. F., Ghofranipour, F., Montazeri, A., Anoosheh, M., Bazargan, M., Mohammadi, E.,...Niknami, S. (2010). Muslim breast cancer survivor spirituality: Coping strategy or health seeking behavior hindrance? Health 
Care for Women International, 31, 88-98.

doi:10.1080/07399330903104516

Harralson, T. L. (2007). Factors influencing delay in seeking treatment for acute ischemic symptoms among lower income, urban women. Heart \& Lung, 36, 96-104. doi:10.1016/j.hrtlng.2006.08.002

Hazewinkel, M., Sprangers, M., Taminiau-Bloem, E., van der Velden, J., Burger, M., \& Roovers, J. (2009). Reasons for not seeking medical help for severe pelvic floor symptoms: A qualitative study in survivors of gynaecological cancer. BJOG, 117, 39-46.

doi:10.1111/j.1471-0528.2009.02411.x

Henderson, C., Evans-Lacko, S., Flach, C., \& Thornicroft, G. (2012). Responses to mental health stigma questions: The importance of social desirability and data collection method. Canadian Journal of Psychiatry, 57(3), 152160.

Henkel, K. E., Brown, K., \& Kalichman, S. C. (2008). AIDS-related stigma in individuals with other stigmatized identities in the USA: A review of layered stigmas. Social and Personality Psychology Compass, 2(4), 1586-1599. doi:10.1111/j.1751-9004.2008.00116.x

Henoch, I., Ploner, A., \& Tishelman, C. (2009). Increasing stringency in symptom cluster research: A methodological exploration of symptom clusters in patients with inoperable lung cancer. Oncology Nursing Forum, 36(6), E282-E292.

Higginson, R. (2008). Women's help-seeking behavior at the onset of myocardial 
infarction. British Journal of Nursing, 17(1), 10-14.

Houlihan, N. G. (2006). Site-specific cancer series: Lung cancer ( $3^{\text {rd }}$ ed.).

Pittsburg, PA: Oncology Nursing Society.

Howell, D., Smith, A. G., \& Roman, E. (2008). Help-seeking behaviour in patients with lymphoma. European Journal of Cancer Care, 17, 394-403. doi:10.1111/j.1365-2354.2007.00897.x

Jemal, A., Bray, F., Center, M. M., Ferlay, J., Ward, E., \& Forman, D. (2011). Global cancer statistics. A Cancer Journal for Clinicians, 61(2), 69-90. Jensen, A. R., Mainz, J., \& Overgaard, J. (2002). Impact of delay on diagnosis and treatment of primary lung cancer. Acta Oncologica, 41(2), 147-152.

Katapodi, M. C., Pierce, P. F., \& Facione, N. C. (2010). Distrust, predisposition to use health services and breast cancer screening: Results from a multicultural community-based survey. International Journal of Nursing Studies, 47(8), 975-983. doi:10.1016/j.jjnurstu.2009.12.014

Kaur, R., Lopez, V., \& Thompson, D. R. (2006). Factors influencing Hong Kong Chinese patients' decision-making in seeking early treatment for acute myocardial infarction. Research in Nursing \& Health, 29, 636-646. doi:10.1002/nur.20171

Kennedy, B. R., Mathis, C. C., \& Woods, A. K. (2007). African Americans and their distrust of the health care system: Healthcare for diverse populations. Journal of Cultural Diversity, 14(2), 56-60.

Khraim, F. M., Scherer, Y. K., Dorn, J. M., \& Carey, M. G. (2009). Predictors of decision delay to seeking health care among Jordanians with acute 
myocardial infarction. Journal of Nursing Scholarship, 41(3), 260-267. doi:10.1111/j.1547-5069.2009.01287.x

Koyi, H., Hillerdal, G., \& Branden, E. (2002). Patient's and doctors' delay in the diagnosis of a chest tumor. Lung Cancer, 35, 53-57.

Knapp, T. R. (1998). Quantitative nursing research. Thousand Oaks, CA: SAGE Publications, Inc.

Kristiansen, C. M., \& Harding, C. M. (1984). The social desirability of preventive health behavior. Public Health Reports, 99(4), 384-388.

Lam, W. W. T., Tsuchiya, M., Chan, M., Chan, S. W. W., Or, A., \& Fielding, R. (2008). Help-seeking patterns in Chinese women with symptoms of breast disease: A qualitative study. Journal of Public Health, 31(1), 59-68. doi:10.1093/pubmed/fdn088

Lauver, D. (1994). Care-seeking behavior with breast cancer symptoms in Caucasian and African-American women. Research in Nursing \& Health, $17,421-431$.

Lebel, S., \& Devins, G. (2008). Stigma in cancer patients whose behavior may have contributed to their disease. Future Oncology, 4, 717-733.

Lee, H. O. (1997). Typical and atypical clinical signs and symptoms of myocardial infarction and delayed seeking of professional care among blacks. American Journal of Critical Care, 6, 7-13.

Lee, J. L., \& Kim, K. S. (2011). The relationships between stigma, distress, and quality of life in patients with lung cancer. Journal of Korean Oncology Nursing, 11(3), 237-246. 
Lekas, H., Siegel, K., \& Leider, J. (2011). Felt and enacted stigma among HIV/HCV-coinfected adults: The impact of stigma layering. Qualitative Health Research, 21(9), 1205-1219. doi:10.1177/1049732311405684

Leprieur, E. G., Labrune, S., Giraud, V., Gendry, T., Cobarzan, D., \& Chinet, T. (2012). Delay between the initial symptoms, the diagnosis and the onset of specific treatment in elderly patients with lung cancer. Clinical Lung Cancer, 13(5), 363-368. doi:10.1016/j.cllc.2011.11.010

Lesneski, L. (2010). Factors influencing treatment delay for patients with acute myocardial infarction. Applied Nursing Research, 23, 185-190. doi:10.1016/j.apnr.2008.09.004

Levealahti, H., Tishelman, C., \& Ohlen, J. (2007). Framing the onset of lung cancer biographically: Narratives of continuity and disruption. PsychoOncology, 16, 466-473. doi:10.1002/pon.1080

Leventhal, H., Nerenz, D., \& Steele, D. J. (1984). IIIness representations and coping with health threats. In Baum, A., Taylor, S. E., \& Singer, J. E. (Eds.) Handbook of psychology and health: Vol IV. Social psychological aspects of health. Hillsdale, NJ: Erlbaum, pp.219-252.

Linsell, L., Forbes, L. J. L., Kapari, M., Burgess, C., Omar, L., Tucker, L., \& Ramirez, A. J. (2009). A randomized controlled trial of an intervention to promote early presentation of breast cancer in older women: Effect on breast cancer awareness. British Journal of Cancer, 101, S40-S48. doi:10.1038/sj.bjc.6605389

LoConte, N. K., Else-Quest, N. M., Eickhoff, J., Hyde, J., \& Schiller, J. H. (2008). 
Assessment of guilt and shame in patients with non-small cell lung cancer compared with patients with breast and prostate cancer. Clinical Lung Cancer, 9(3), 171-178. doi:10.3816/CLC.2008.n.026

Lovgren, M., Levealahti, H., Tishelman, C., Runesdotter, S., Hamberg, K., \& Koyi, H. (2008). Time spans from first symptom to treatment in patients with lung cancer: The influence of symptoms and demographic characteristics. Acta Oncologica, 47, 397-405.

doi:10.1080/02841860701592392

Lovlien, M., Schei, B., \& Hole, T. (2007). Prehospital delay, contributing aspects and responses to symptoms among Norwegian women and men with first time acute myocardial infarction. European Journal of Cardiovascular Nursing, 6, 308-313. doi:10.1016/j.ejcnurse.2007.03.002

Lowndes, C. M., Jayachandran, A. A., Banandur, P., Ramesh, B. M., Washington, R., Sangameshwar, B. M.,...Alary, M. (2012). Polling booth surveys: A novel approach for reducing social desirability bias in HIVrelated behavioural surveys in resource-poor settings. AIDS Behavior, 16, 1054-1062.

Macleod, U., Mitchell, E. D., Burgess, C., Macdonald, S., \& Ramirez, A. J. (2009). Risk factors for delayed presentation and referral of symptomatic cancer: Evidence for common cancers. British Journal of Cancer, 101, S92-S101. doi:10.1038/sj.bjc.6605398

Marlow, L., Waller, J., \& Wardle, J. (2010). Variation in blame attributions across different cancer types. Cancer, Epidemiology, Biomarkers, and 
Prevention, 19, 1799-1805. doi:10.1158/1055-9965.EPI-09-1298

Mascarenhas, O. A., Cardozo, L. J., Afonso, N. M., Siddique, M., Steinberg, J., Lepczyk, M., \& Aranha, A. N. (2006). Hypothesized predictors of patientphysician trust and distrust in the elderly: Implications for health and disease management. Clinical Interventions in Aging, 1(2), 175-188.

Mason, O. J., \& Strauss, K. (2004a). Studying help-seeking for testicular cancer: Some lessons from the literature (Part 2). International Journal of Men's Health, 3(2), 111-127.

Mason, O. J., \& Strauss, K. (2004b). Testicular cancer: Passage through the help-seeking process for a cohort of U.K. men (Part 1). International Journal of Men's Health, 3(2), 93-110.

McAlearney, A. S., Oliveri, J. M., Post, D. M., Song, P. H., Jacobs, E., Waibel, J.,...Paskett, E. D. (2012). Trust and distrust among Appalachian women regarding cervical cancer screening: A qualitative study. Patient and Education Counseling, 86, 120-126. doi:10.1016/j.pec.2011.02.023 McSweeney, J. C., Lefler, L. L., Fischer, E. P., Naylor, A. J., \& Evans, L. K. (2007). Women's prehospital delay associated with myocardial infarction: Does race really matter? Journal of Cardiovascular Nursing, 22(4), 279285.

Meischke, H., Mitchell, P., Zapka, J., Goff, D. C., Smith, K., Henwood, D.,...Taylor, J. (2000). The emergency department experience of chest patients and their intention to delay care seeking for acute myocardial infarction. Progress in Cardiovascular Nursing, 15(2), 50-57 \& 70. 
Minino, A. M., Murphy, S. L., Xu, J., \& Kochanek, K. D. Deaths: Final data for 2008. National Vital Statistics Reports, 59(10). Hyattsville, MD: National Center for Health Statistics, 2011.

Mohammed, N., Kestin, L. L., Grills, I. S., Battu, M., Fitch, D. L., Wong, C. O., Margolis, J. H., Chmielewski, G. W., \& Welsh, R. J. (2011). Rapid disease progression with delay in treatment of non-small-cell lung cancer. International Journal of Radiation Oncology, Biology, \& Physics, 79(2), 466-472. doi:10.1016/j.ijrobp.2009.11.029

Molassiotis, A., Wilson, B., Brunton, L., \& Chandler, C. (2010). Mapping patients' experiences from initial change in health to cancer diagnosis: A qualitative exploration of patient and system factors mediating this process. European Journal of Cancer Care, 19(1), 98-109. doi:10.1111/j.1365-2354.2008.01020.x

Moloczij, N., McPherson, K. M., Smith, J. F., \& Kayes, N. M. (2008). Helpseeking at the time of stroke: Stroke survivors' perspectives on their decisions. Health and Social Care in the Community, 16(5), 501-510. doi: 10.1111/j.1365-2524.2008.00771.x

Moody, A., Muers, M., \& Forman, D. Delays in managing lung cancer. Thorax, $59,1-3$

Mor, V., Masterson-Allen, S., Goldberg, R., Guadagnoli, E., \& Wool, M. S. (1990). Pre-diagnostic symptom recognition and help seeking among cancer patients. Journal of Community Health, 15(4), 253-266.

Moseley, K. L., Freed, G. L., Bullard C. M., \& Goold, S. D. (2007). Measuring 
African-American parents' cultural mistrust while in a healthcare setting: A pilot study. Journal of the National Medical Association, 99, 115-121.

Moser, D. K., Kimble, L. P., Alberts, M. J., Alonzo, A., Croft, J. B., Dracup, K., . . . Zerwic, J. J. (2006). Reducing delay in seeking treatment by patients with acute coronary syndrome and stroke: A scientific statement from the American Heart Association Council on Cardiovascular Nursing and Stroke Council. Circulation, 114, 168-182.

Muzzin, L. J., Anderson, N. J., Figueredo, A. T., \& Gudelis, S. O. (1994). The experience of cancer. Social Science \& Medicine, 38(9), 1201-1208.

Nadler, A. (1987). Determinants of help seeking behavior: The effects of helper's similarity, task centrality and recipient's self esteem. European Journal of Social Psychology, 17, 57-67.

O'Mahoney, M., \& Hegarty, J. (2009). Help seeking for cancer symptoms: A review of the literature. Oncology Nursing Forum, 36(4), E178-E184.

O’Mahoney, M., Hegarty, J., \& McCarthy, G. (2011). Women's help seeking behavior for self discovered breast cancer symptoms. European Journal of Oncology Nursing, 15, 410-418. doi:10.1016/j.ejon.2010.10.011

Pack, G. T., \& Gallo, J. S. (1938). The culpability for delay in the treatment of cancer. American Journal of Cancer, 33, 443-462.

Pallant, J. (2010). SPSS survival manual (4 ${ }^{\text {th }}$ ed.). New York, NY: McGraw-Hill. Perkins-Porras, L., Whitehead, D. L., Strike, P. C., \& Steptoe, A. (2009). Prehospital delay in patients with acute coronary syndrome: Factors associated with patient decision time and home-to-hospital delay. 
European Journal of Cardiovascular Nursing, 8, 26-33.

doi:10.1016/j.ejcnurse.2008.05.001

Peterson, S. J., \& Bredow, T. S. (2009). Middle range theories: Application to nursing research $\left(2^{\text {nd }}\right.$ ed.) Philadelphia, PA: Wolters Kluwer Health Lippincott Williams \& Wilkins.

Plowden, K. O. (2005). A theoretical approach to understanding black men's health-seeking behavior. The Journal of Theory Construction \& Testing, 7(1), 27-31.

Polit, D. F., \& Beck, C. T. (2004). Nursing research: Principles and methods $\left(7^{\text {th }}\right.$ ed.). Philadelphia, PA: Lippincott Williams \& Wilkins.

Quinn, J. R. (2005). Delay in seeking care for symptoms of acute myocardial infarction: Applying a theoretical model. Research in Nursing \& Health, 28, 283-294. doi:10.1002/nur.20086

Raleigh, Z. T. (2010). A biopsychosocial perspective on the experience of lung cancer. Journal of Psychosocial Oncology, 28(1), 116-125. doi:10.1080/07347330903438990

Ramirez, A. J., Westcombe, A. M., Burgess, C. C., Sutton, S., Littlejohns, P., \& Richards, M. A. (1999). Factors predicting delayed presentation of symptomatic breast cancer: A systematic review. Lancet, 353(9159), $1127-1131$.

Ravenell, J. E., Whitaker, E. E., \& Johnson, W. E. (2008). According to him: Barriers to healthcare among African-American men. Journal of the National Medical Association, 100, 1153-1160. 
Reifenstein, K. (2007). Care-seeking behaviors of African American women with breast cancer symptoms. Research in Nursing \& Health, 30(5), 542-557. doi:10.1002/nur.20246

Ristvedt, S. L., \& Trinkhaus, K. M. (2005). Psychological factors related to delay in consultation for cancer symptoms. Psycho-oncology, 14, 339-350. doi:10.1002/pon.850

Rose, A., Peters, N., Shea, J. A., \& Armstrong, K. (2004). Development and testing of the health care system distrust scale. Journal of General Internal Medicine, 19, 57-63.

Russell, E., Robinson, D. H. Z., Thompson, N. J., Perryman, J. P., \& Arriola, K. R. J. (2012). Distrust in the healthcare system and organ donation intentions among African Americans. Journal of Community Health, 37, 40-47. doi:10.1007/s10900-011-9413-3

Salomaa, E., Sallinen, S., Hiekkanen, H., \& Liippo, K. (2005). Delays in the diagnosis and treatment of lung cancer. Chest, 128, 2282-2288. doi:10.1378/chest.128.4.2282

Sarna, L., \& Brecht, M. L. (1997). Dimensions of symptom distress in women with advanced lung cancer: A factor analysis. Heart \& Lung, 26(1), 23-30.

Schenker, Y., White, D. B., Asch, D. A., \& Kahn, J. M. (2012). Health-care system distrust in the intensive care unit. Journal of Critical Care, 27, 3-10. doi:10.1016/j.jcrc.2011.04.006

Sethi, T. K., El-Ghamry, M. N., \& Kloecker, G. H. (2012). Radon and lung cancer. Clinical Advances in Hematology \& Oncology, 10(3), 157-164. 
Shaikh, B. T., Haran, D., \& Hatcher, J. (2008). Women's social position and health-seeking behaviors: Is the health care system accessible and responsive in Pakistan? Health Care for Women International, 29(8-9), 945-959. doi:10.1080/07399330802380506

Shaw, C., Brittain, K., Tansey, R., \& Williams, K. (2008). How people decide to seek health care: A qualitative study. International Journal of Nursing Studies, 45, 1516-1524. doi:10.1016/j.jnurstu.2007.11.005

Shea, J. A., Micco, E., Dean, L. T., McMurphy, S., Schwartz, J. S., \& Armstrong, K. (2008). Development of the revised health care system distrust scale. Journal of General Internal Medicine, 23(6), 727-732. doi:10.1007/s11606-008-0575-3

Sheikh, I., \& Ogden, J. (1998). The role of knowledge and beliefs in help seeking behaviour for cancer: A quantitative and qualitative approach. Patient Education and Counseling, 35, 35-42.

Shoff, C., \& Yang, T. (2012). Untangling the associations among distrust, race, and neighborhood social environment: A social disorganization perspective, Social Science \& Medicine, 74(9), 1342-1352. doi:10.1016/j.socscimed.2012.01.012

Singh, H., Hirani, K., Kadiyala, H., Rudomiotov, O., Davis, T., Khan, M. M., \& Wahls, T. L. (2010). Characteristics and predictors of missed opportunities in lung cancer diagnosis: An electronic health record-based study. Journal of Clinical Oncology, 28(20), 3307-3315. doi:10.1200/JCO.2009.25.6636

Sjostrom-Strand, A., \& Fridlund, B. (2008). Women's descriptions of symptoms 
and delay reasons in seeking medical care at the time of a first myocardial infarction: A qualitative study. International Journal of Nursing Studies, 45, 1003-1010. doi:10.1016/j.jinurstu.2007.07.004

Spiro, S. G., Gould, M. K., \& Colice, G. L. (2007). Initial evaluation of the patient with lung cancer: Symptoms, signs, laboratory tests, and paraneoplastic syndromes: AACP evidenced-based clinical practice guidelines $\left(2^{\text {nd }}\right.$ edition). CHEST, 132, 149S-160S. doi:10.1378/chest.07-1358

SPSS Statistics for Maclntosh 20 [Computer software]. (2012). Chicago, IL.

Stahly, G. B. (1989). Psychological aspects of the stigma of cancer: An overview. Journal of Psychosocial Oncology, 6(3), 3-27.

Stevens, J. P. (2009). Applied multivariate statistics for the social sciences $\left(5^{\text {th }}\right.$ ed.). New York, NY: Routledge Taylor \& Francis Group.

Strahan, R., \& Gerbasi, K. C. (1972). Short, homogenous versions of the MarlowCrowne Social Desirability Scale. Journal of Clinical Psychology, 28(2), 191-193.

Stuber, J., Galea, S., \& Link, B. G. (2008). Smoking and the emergence of a stigmatized social status. Social Science \& Medicine, 67, 420-430. doi:10.1016/j.socscimed.2008.03.010

Stutterheim, S. E., Bos, A. E. R., Pryor, J. B., Brands, R., Liebregts, M., \& Schaalma, H. P. (2011). Psychological and social correlates of HIV status disclosure: The significance of stigma visibility. AIDS Education and Prevention, 23(4), 382-392.

Surveillance, Epidemiology, and End Results (SEER) Program 
(www.seer.cancer.gov) Research Data (2011), National Cancer Institute, DCCPS, Surveillance Research Program, Surveillance Systems Branch. Tabachnick, B. G., \& Fidell, L. S. (2001). Using multivariate statistics $\left(4^{\text {th }}\right.$ ed.). Boston: Allyn \& Bacon.

Taib, N. A., Yip, C., \& Low, W. (2011). Recognizing symptoms of breast cancer as a reason for delayed presentation in Asian women: The psycho-sociocultural model for breast symptom appraisal: Opportunities for intervention. Asian Pacific Journal of Cancer Prevention, 12, 1601-1608.

Thom, D. H., Kravitz, R. L., Bell, R. A., Krupat, E., \& Azari, R. (2002). Patient trust in the physician: Relationship to patient requests. Family Practice, $19,476-483$.

Thom, D. H., Ribisl, K. M., Stewart, A. L., \& Luke, D. A. (1999). Further validation and reliability testing of the Trust in Physician Scale: The Stanford Trust Study Physicians. Medical Care, 37, 510-517.

Thun, M. J., Henley, S. J., Burns, D., Jemal, A., Shanks, T. G., \& Calle, E. E. (2006). Lung cancer death rates in lifelong nonsmokers. Journal of the National Cancer Institute, 98(10), 691-699. doi:10.1093/jnci/djj187

Tod, A. M., Craven, J., \& Allmark, P. (2008). Diagnostic delay in lung cancer: A qualitative study. Journal of Advanced Nursing, 61(3), 336-343. doi:10.1111/j.1365-2648.2007.04542.x

Tod, A. M., \& Joanne, R. (2010). Overcoming delay in the diagnosis of lung cancer: A qualitative study. Nursing Standard, 24(31), 35-43.

Tullmann, D. F., Haugh, K. H., Dracup, K. A., \& Bourguignon, C. (2007). A 
randomized controlled trial to reduce delay in older adults seeking help for symptoms of acute myocardial infarction. Research in Nursing \& Health, 30, 485-497. doi:10.1002/nur.20245

U. S. Census Bureau (2013, March 11). State \& County Quickfacts: Jefferson County, KY Retrieved May 14, 2013 from http://quickfacts.census.gov/qfd/states/21/21111.html.

Van Brakel, W. H. (2006). Measuring health-related stigma: A literature review. Psychology, Health, \& Medicine, 11(3), 307-334. doi:10.1080/13548500600595160

Waljee, J. F., Hu, E. S., Newman, L. A., \& Alderman, A. K. (2008). Correlates of patient satisfaction and provider trust after breast-conserving surgery. Cancer, 112(8), 1679-1687.

Wall, C., Glenn, S., \& Poole, H. (2011). Experiences prior to diagnosis of non-Hodgkin lymphoma: A phenomenological study. Journal of Advanced Nursing, 67(11), 2363-2372. doi:10.1111/j.1365-2648.2011.05657.x Waller, J., Robb, K., Stubbings, S., Ramirez, A., Macleod, U., Austoker, J., . . . Wardle, J. (2009). Awareness of cancer symptoms and anticipated help seeking among ethnic minority groups in England. British Journal of Cancer, 101, S24-S30. doi:10.1038/sj.bjc.6605387

Wang, S. Y., Tsai, C. M., Chen, B. C., Lin, C. H., \& Lin, C. C. (2008). Symptom clusters and relationships to symptom interference with daily life in Taiwanese lung cancer patients. Journal of Pain Symptom Management, 35(3), 258-266. 
Wang, X. S., Fairclough D. L., Liao, Z., Komaki, R., Change, J. Y., Mobley, G. M., \& Cleeland, C. S. (2006). Longitudinal study of the relationship between chemoradiation therapy for non-small-cell lung cancer and patient symptoms. Journal of Clinical Oncology, 24(27), 4485-4491.

doi:10.1200/JCO.2006.07.1126

Wasserman, J., Flannery, M. A., \& Clair, J. M. (2007). Raising the ivory tower: The production of knowledge and distrust of medicine among African Americans. Journal of Medical Ethics, 33(3), 177-180.

doi:10.1136/jme.2006.016329

Weiss, M., \& Ramakrishna, J. (2006). Stigma interventions and research for international health. Lancet, 367, 536-538.

Wolitski, R. J., Pals, S. L., Kidder, D. P., Courtenay-Quirk, C., \& Holtgrave, D. R. (2009). The effects of HIV stigma on health, disclosure of HIV status, and risk behavior of homeless and unstably housed persons living with HIV, AIDS Behavior, 13(6), 1222-1232. doi:10.1007/s10461-008-9455-4

Wyatt, P., \& Ratner, P. (2004). Evaluating treatment-seeking for acute myocardial infarction in women. Canadian Journal of Cardiovascular Nursing, 14(1), 39-45.

Yang, T., \& Matthews, S. A. (2012). Understanding the non-stationary associations between distrust of the health care system, health conditions, and self-rated health in the elderly: A geographically weighted regression approach. Health \& Place, 18, 576-585.

doi:10.1016/j.healthplace.2012.01.007 
Yang, T., Matthews, S. A., \& Hillemeier, M. M. (2011). Effect of health care system distrust on breast and cervical cancer screening in Philadelphia, Pennsylvania. Research and Practice, 101(7), 1297-1305. doi:10.2105/AJPH.2010.300061

Zegrean, M., Fox-Wasylyshyn, S. M., \& El-Masri, M. M. (2009). Alternative coping strategies and decision delay in seeking care for acute myocardial infarction. Journal of Cardiovascular Nursing, 24(2), 151-155.

Zerwic, J., Hwang, S. Y., \& Tucco, L. (2007). Interpretation of symptoms and delay in seeking treatment by patients who have had a stroke: Exploratory study. Heart \& Lung, 36(1), 25-34.

Zhou, Y. R. (2009). Help-seeking in the context of AIDS stigma: Understanding the healthcare needs of people with HIVIAIDS in China. Health and Social Care in the Community, 17(2), 202-208.

doi:10.111/j.1365-2524.2008.00820.x 


\title{
Appendix A
}

\section{Approval Letters}

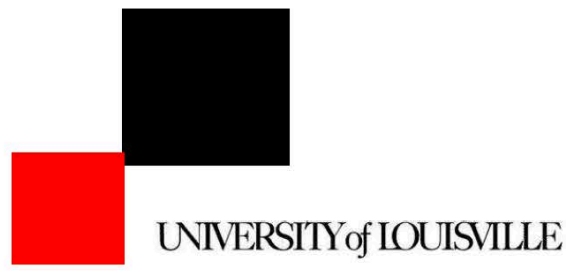

\author{
INSTITUTIONAL REVIEW BOARDS \\ University of Louisville \\ MedCenter One, Suite 200 \\ 501 E. Broadway \\ Louisville, Kentucky 40202-1798 \\ Fax: $\quad 502-852-2164$
}

\author{
To: Hermann, Carla \\ From: $\quad$ The University of Louisville Institutional Review Board (IRB) \\ Date: $\quad$ Friday, November 30, 2012 \\ Subject: Approval Letter \\ Tracking \#: 12.0523 \\ Title: $\quad$ Variables that Influence the Timing of Help-Seeking Behavior in Lung \\ Cancer Patients \\ Approval 11/14/2012 12:00:00 AM \\ Date: \\ Expiration $\quad 11 / 13 / 2013$ 12:00:00 AM \\ Date:
}

This study was reviewed on $11 / 14 / 2012$ by the chair/vice chair of the Institutional Review Board and approved through the Expedited Review Procedure, according to 45 CFR 46.110(b), since this study falls under Expedited Category (7) Research on individual or group characteristics or behavior (including, but not limited to, research on perception, cognition, motivation, identity, language, communication, cultural beliefs or practices, and social behavior) or research employing survey, interview, oral history, focus group, program evaluation, human factors evaluation, or quality assurance methodologies.

This study was also approved through 45 CFR 46.117 (c), which means that an IRB may waive the requirement for the investigator to obtain a signed informed consent form for some or all subjects if it finds either:

- That the only record linking the subject and the research would be the consent document and the principal risk would be potential harm resulting from a breach of confidentiality. Each subject will be asked whether the subject wants documentation linking the subject with the research, and the subject's wishes will govern; or

- That the research presents no more than minimal risk of harm to subjects and involves no procedures for which written consent is normally required outside of the research context.

The following items have been approved:

- v10262012 Carter Harris Dissertation Research Protocol, dated October 26, 
2012

- Recruitment Flyer Carter Harris Dissertation Study, not dated

- Carter Harris Dissertation Study Preamble, dated October 15, 2012

This study now has final IRB approval from $11 / 14 / 2012$ through $11 / 13 / 2013$. You should complete and return the Progress Report/Continuation Request Form EIGHT weeks prior to this date in order to ensure that no lapse in approval occurs. The committee will be advised of this action at their next full board meeting.

Site Approval

If this study will take place at an affiliated research institution, such as Jewish Hospital/St Marys Hospital, Norton Healthcare, or University of Louisville Hospital, permission to use the site of the affiliated institution may be necessary before the research may begin. If this study will take place outside of the University of Louisville Campuses, permission from the organization should be obtained before the research may begin. Failure to obtain this permission may result in a delay in the start of your research.

Privacy \& Encryption Statement

The University of Louisville's Privacy and Encryption Policy requires such information as identifiable medical and health records: credit card, bank account and other personal financial information; social security numbers; proprietary research data; dates of birth (when combined with name, address and/or phone numbers) to be encrypted. For additional information: http://security.louisville.edu/PolStds/ISO/PS018.htm.

1099 Information (If Applicable)

As a reminder, in compliance with University policies and Internal Revenue Service code, all payments (including checks, gift cards, and gift certificates) to research subjects must be reported to the University Controller's Office. Petty Cash payments must also be monitored by the issuing department and reported to the Controller's Office. Before issuing compensation, each research subject must complete a $\mathrm{W}-9$ form.

For additional information, please contact the Controller's Office at 852-8237 or contro ॥@louisville.edu.

The following is a NEW link to an Instruction Sheet for BRAAN2 "How to Locate Stamped/Approved Documents in BRAAN2" if your item was submitted on or after $5 / 17 / 10$ :

http://louisville.edu/research/braan2/help/ApprovedDocs.pdf/view

Please begin using your approved (stamped) document(s) at this time. The previous versions are no longer valid. If you need assistance in accessing any of the study documents, please feel free to contact our office at (502) 852-5188. You may also email our service account at hsppofc@louisville edu for assistance.

Best wishes for a successful study. If you have any questions please contact the 
Thank you.

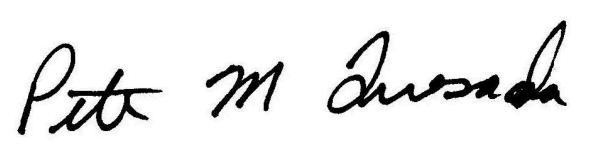

Board Designee: Quesada, Peter

Once you begin your human subject research the following regulations apply:

1. Unanticipated problems or serious adverse events encountered in this research study must be reported to the IRB within five (5) work days.

2. Any modifications to the study protocol or informed consent form must be reviewed and approved by the IRB prior to implementation.

3. You may not use a modified informed consent form until it has been approved and validated by the IRB.

4. Please note that the IRB operates in accordance with laws and regulations of the United States and guidance provided by the Office of Human Research Protection (OHRP), the Food and Drug Administration (FDA), the Office of Civil Rights (OCR) and other Federal and State Agencies when applicable.

5. You should complete and SUBMIT the Continuation Request Form eight weeks prior to this date in order to ensure that no lapse in approval occurs.

Letter Sent By: Block, Sherry, 11/30/2012 3:04 PM 


\section{UniversityHospital}

\section{Lofoud menter of}

November 21, 2012

Re: Variables that Influence the Timing of Help-Seeking Behavior in Lung Cancer Patients

Dr. Carla Hermann PhD, RN

University of Louisville School of Nursing

Health Sciences Campus, K-Wing

555 South Floyd Street

Suite 3019

Louisville, KY 40202

Dear Dr. Hermann and Ms. Carter-Harris,

On November 21, 2012 the Nursing and Interdisciplinary Research Committee (NIRC) conducted a scientific review of your proposed research study. The committee members determined that there were no threats to internal and external validity of the study, and that the study had the potential to advance scientific knowledge in the field. In addition, the study does not appear to have an adverse fiscal impact on any nursing unit. As a means of follow-up, the NIRC would appreciate an update on you progress the last month of each quarter at their monthly business meeting.

The next step in the approval process is submission in the BRAAN2 system. You may access the BRAAN2 system online at the following web address: http://louisville.edu/research/braan2

Once the BRAAN2 submission is complete an electronic copy of your proposal will be received and reviewed by the Human Subjects Protection Program at the University of Louisville. You may contact them at hsppofc@louisville.edu or (502) 852-5188. It will also be reviewed by the Research Integrity Office at University of Louisville Medical Center to determine that the study does not violate any federal regulations. Please contact Stephanie Deetsch, the Research Compliance Coordinator, for any questions related to that process at stephare $@$ ulh.org or (502) 562-3737).

Thank you for advancing the nursing research enterprise at University of Louisville Medical Center.

\section{Sincerely,

Reetta Stikes, MSN, RNC-NIC, CLC

Chair, Nursing and Interdisciplinary Research Committee

University of Louisville Medical Center

(502) $562-3710$

Junest@ulh.org

CC: Stephanie Deetsch 
UNIVERSTTY of IOUSSVILE:

Health Sciences Center

JAMES GRAHAM BROWN CANCER CENTER

CLINTC AL SCIENTIFIC REVTEW COMOUTTE

529 South Yackson Sitreet

Room 4 ti

Phone 5025623.47
Fax 5022178273

Louisville, KY 40202

\section{MEMORANDUM}

TO:

FROM:

DATE:

SUBJECT:

RECOMMENDATION:
Carla Hermann, Ph.D., RN

Donald M. Miller, M. D., Ph. D. CSRC Chairman

December 7, 2012
DMM

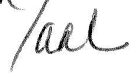

Variables that Influence the Timing of Help-Seeking Behavior in Lung Cancer Patients

Expedited Approval

CSRC0679 - Your investigator initiated protocol "Variables that Influence the Timing of Help-Seeking in Lung Cancer Patients" was reviewed by the Clinical Scientific Research Committee Chairman, Donald M. Miller, M.D, Ph.D., for expedited approval. Your protocol has been approved for activation.

Thank you for submitting this protocol. 


\section{UofL HealthCare University Hospital}

November 20, 2012

530 South Jackson Street

Carla Hermann PhD, RN

Louisville, Kentucky 40202

c/o Lisa Harris

Nursing Administration

$555 \mathrm{~S}$. Floyd St, K Bldg

Louisville, KY 40202

IRB\#12.0523: Variables that Influence the Timing of Help-Seeking Behavior in Lung Cancer Patients

Dear Dr. Hermann:

The $U$ of L Hospital Research Integrity Office (ULH-RIO) has reviewed and approved your application to conduct the above entitled research study in our facility. Your RIO tracking number for this study is RIO1032.

Please be advised of the following:

1. You will need to present this letter to the HIM department in order to get charts pulled for research purposes.

2. The Health Information Management (HIM) Department should be provided as much advanced notice as possible for chart pull, at least two weeks.

3. No matter how many medical records are reviewed, whether 1 or 100 , you and your coinvestigators are responsible to ensure that an IRB approved Complete Waiver is placed in each patient's chart, whether a paper chart or an electronic chart.

4. There is to be no deviation from the approved research protocol without additional approval from the ULH Research Integrity Office.

5. If medical students are added to this study, they must undergo a ULH Research Credentialing Process through our office before they are allowed to review charts or work with data. Please contact our office to begin this process.

6. At study termination, you are responsible for submitting a study summary report to our office listing the total number of charts pulled and the date that the study was closed.

Your attention to the above required processes will help to ensure the safety of our patients and the hospital. Declaration:

I hereby certify that the review and approval of the above mentioned study by the ULH RIO was:

- Devoid of any financial, social or professional conflict of interest, and

- Was not influenced in any way by the Investigators or the research study team members.

Thank you for choosing to conduct your research at the ULH/JGBCC. Should you have any questions or concerns, please do not hesitate to me at 562-3737.

Sincerely,

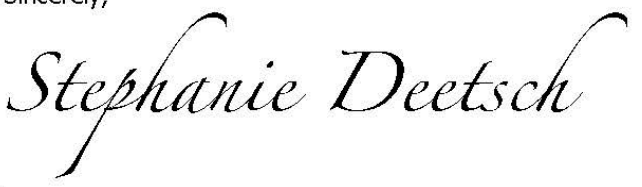

Stephanie Deetsch, CCRP, CHRC

Manager, Research Regulatory Affairs \& Compliance

Research Integrity Office

Study \# 12.0523 Hermann PhD RN Input from Expedited Review Committee, ULH RRC Page 1 of 3 
Cc: Walter Zupances, Director of HIM; Claire Metzger, Manager, HIM

Study \# 12.0523 Hermann PhD RN Input from Expedited Review Committee, ULH RRC Page 2 of 3 
Study \# 12.0523 EXPEDITED INITIAL REVIEW

PI: Carla Hermann PhD RN

Today's date: November 15, 2012

Drugs: none

Subjects: Pts who were diagnosed with lung cancer who had symptoms before their diagnosis

Intervention: Questionnaire and Interview study

Phase: Exploratory

Sponsor: Investigator Initiated

\# Local Subjects: 92 (110)

Sites: ULH and Baptist East

Input from ULH Expedited Research Review Committee

Recommendation of Expedited Research Review Committee: Approve as submitted.

Research Credentialing: Please make sure the investigators and research personnel have updated research credentialing at ULH through the Research Integrity Office. Call Stephanie Deetsch at 562-3737 for more information. Please Note: If a student is added to this study, the student must undergo a research credentialing process through our office before interacting with research data.

IRB Status: In expedited review

Overall Questions/Concerns for the Investigator:

This is an excellent study and the research documents are extremely polished. Truly exemplary.

Research Protocol, October 26, 2012 version: No questions or concerns.

Questionnaires, Fact Sheet, and Advertisement: No questions or concerns.

Preamble, dated October 15, 2012: No questions or concerns. Exemplary.

IRB Protocol, Version 2: No questions or concerns.

HIPAA DOCUMENTS: none needed

Billing Compliance Table: Not needed. 


\section{BAP:IST HOSPITAL EAST \\ Feel better.}

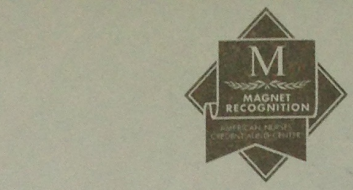

4000 Kresge Way

Louisville, KY 40207

502-897-8100

December 20, 2012

Lisa Carter-Harris, MSN, APRN, NP-C

Doctoral Candidate, School of Nursing

4031 Viewcrest Loop

Floyds Knobs, IN 47119

Dear Lisa:

This letter is confirmation that your research proposal, "Variables that Influence the Timing of Help-Seeking Behavior in Lung Cancer Patients" has been approved by the Baptist Hospital East Nursing Research Council.

We ask that you keep the Research Council apprised of how your study is progressing. Also, at the completion of your study, you will need to submit a copy of the study results for our records, provide a presentation of the results to the Research Council, and write a summary of the study and results for the internal nursing Stethoscoop publication.

As already mentioned in my email to you, Laura Mitchell, 896-7490 will be your contact for getting started.

Please feel free to call me at $896-7456$ or email me if you have any questions. Good luck with your study.

We look forward to you sharing the results.

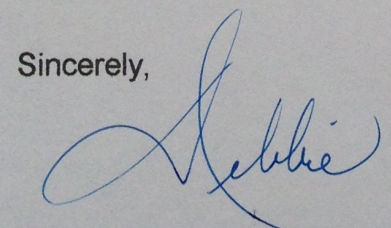

Debbie Nelson, MSN, RN, CRRN

Director of Rehabilitation and Behavioral Health

Chair, Nursing Research Council Proposal Review Subgroup 
Appendix B

IRB-approved Recruitment Flyer

\title{
Do you have lung cancer?
}

\author{
Are you 22 years or older? \\ Do you have lung cancer? \\ Can you speak and understand English?
}

- If you answered YES, I would like to talk with you. This study involves:

One 45 to 60 minute meeting that involves completing 3 paper and pencil surveys and a short interview with the researcher

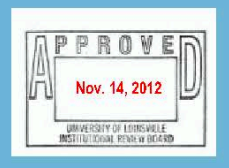

This is a Dissertation Study by Lisa Carter-Harris, PhD Student \& Carla Hermann, PhD, Principal Investigator (Faculty Mentor) University of Louisville School of Nursing

For more information about the study, please contact Lisa CarterHarris directly at 502.541.2405 or lisacarter.harris@louisville.edu

\begin{tabular}{|c|c|c|c|c|c|c|c|c|c|}
\hline 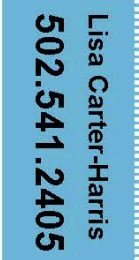 & 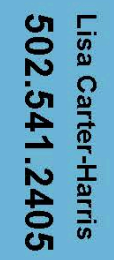 & 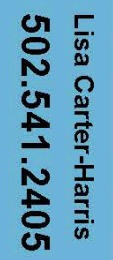 & 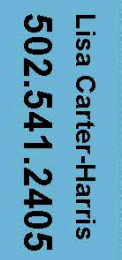 & 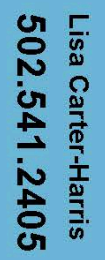 & 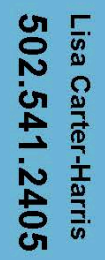 & 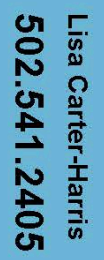 & 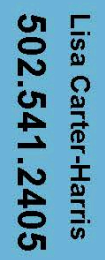 & 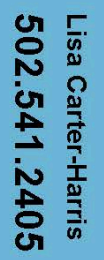 & 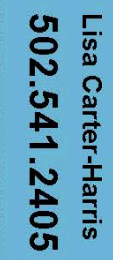 \\
\hline
\end{tabular}




\title{
Appendix C
}

\section{Study Preamble}

\begin{abstract}
UNIVERSITY OF LOUISVILLE
INSTITUTIONAL REVIEW BOARD

Date Approved 11/14/2012Valid Thru 11/13/2013

Variables that Influence the Timing of Help-Seeking Behavior in Lung Cancer Patients
\end{abstract}

October 15,2012

Dear Lung Cancer Patient:

You are being invited to participate in a research study by answering the attached survey about your feelings, medical problems, social and educational situation, and smoking history before you were diagnosed with lung cancer. You are also invited to participate in an oral interview about these same topics. The purpose of this study is to evaluate what motivates a person to seek help when they are ill, specifically, when they are later found to have lung cancer. Your total participation will be about 1 hour. It will take 30 minutes to complete the interview and another 30 minutes to complete the pen and paper questionnaire. If you are tired, we can reschedule for another clinic date. Though unlikely, risks to you include possibly feeling tired, bored, or frustrated from answering questions. You do not have to answer any questions that make you uncomfortable. The information collected may not benefit you directly. The information learned in this study may be helpful to others. The information you provide will help researchers understand the factors that cause a patient with lung cancer to seek health care. Your completed survey and interview responses will be stored at the University of Louisville School of Nursing on a password protected encrypted computer. Paper documents will be kept in a locked filing cabinet.

Individuals from the University of Louisville School of Nursing, the Institutional Review Board (IRB), the Human Subjects Protection Program Office (HSPPO), 
and other regulatory agencies may inspect these records. In all other respects, however, the data will be held in confidence to the extent permitted by law. Should the data be published, your identity will not be disclosed.

Taking part in this study is voluntary. By completing this survey and by completing the interview, you agree to take part in this research study. You may choose not to take part at all. If you decide to be in this study, you may stop taking part at any time. If you decide not to be in this study or if you stop taking part at any time, you will not lose any benefits for which you may qualify.

If you have any questions, concerns, or complaints about the research study, please contact: Carla Hermann, PhD, RN, phone 502-852-8397 or Lisa CarterHarris, MSN, APRN at 502-541-2405.

If you have any questions about your rights as a research subject, you may call the Human Subjects Protection Program Office at (502) 852-5188. You can discuss any questions about your rights as a research subject, in private, with a member of the Institutional Review Board (IRB). You may also call this number if you have other questions about the research, and you cannot reach the research staff, or want to talk to someone else. The IRB is an independent committee made up of people from the University community, staff of the institutions, as well as people from the community not connected with these institutions. The IRB has reviewed this research study.

If you have concerns or complaints about the research or research staff and you do not wish to give your name, you may call 1-877-852-1167. This is a 24 hour hot line answered by people who do not work at the University of Louisville.

Sincerely,

Carla Hermann, PhD, RN

Phone: 502-852-8397
Lisa Carter-Harris, MSN, APRN

$P h D$ in Nursing Student

Phone: $\quad$ 502-541-2405 


\section{Appendix D}

Revised Healthcare System Distrust Scale

Study Participant \#

\section{RHCSDS}

You will find below a series of statements about your opinion of the health care system in general. When we refer to the health care system, we mean hospitals, health insurance companies, and medical research. For each statement below, please circle the number ( 1 to 5 ) that describes you best.

\begin{tabular}{|c|c|c|c|c|c|}
\hline & $\begin{array}{l}\text { Strongly } \\
\text { Agree }\end{array}$ & Agree & Not Sure & Disagree & $\begin{array}{l}\text { Strongly } \\
\text { Disagree }\end{array}$ \\
\hline $\begin{array}{l}\text { 1. The Health Care System does } \\
\text { its best to make patients' } \\
\text { health better. }\end{array}$ & 1 & 2 & 3 & 4 & 5 \\
\hline $\begin{array}{l}\text { 2. The Health Care System } \\
\text { covers up its mistakes. }\end{array}$ & 1 & 2 & 3 & 4 & 5 \\
\hline $\begin{array}{l}\text { 3. Patients receive high quality } \\
\text { medical care from the Health } \\
\text { Care System. }\end{array}$ & 1 & 2 & 3 & 4 & 5 \\
\hline $\begin{array}{l}\text { 4. The Health Care System } \\
\text { makes too many mistakes. }\end{array}$ & 1 & 2 & 3 & 4 & 5 \\
\hline $\begin{array}{l}\text { 5. The Health Care System puts } \\
\text { making money above } \\
\text { patients' needs. }\end{array}$ & 1 & 2 & 3 & 4 & 5 \\
\hline $\begin{array}{l}\text { 6. The Health Care System } \\
\text { gives excellent medical care. }\end{array}$ & 1 & 2 & 3 & 4 & 5 \\
\hline $\begin{array}{l}\text { 7. Patients get the same medical } \\
\text { treatment from the Health } \\
\text { Care System, no matter what } \\
\text { the patient's race or ethnicity. }\end{array}$ & 1 & 2 & 3 & 4 & 5 \\
\hline $\begin{array}{l}\text { 8. The Health Care System lies } \\
\text { to make money. }\end{array}$ & 1 & 2 & 3 & 4 & 5 \\
\hline $\begin{array}{l}\text { 9. The Health Care System } \\
\text { experiments on patients } \\
\text { without them knowing. }\end{array}$ & 1 & 2 & 3 & 4 & 5 \\
\hline
\end{tabular}




\section{Appendix $\mathrm{E}$}

\section{Cataldo Lung Cancer Stigma Scale}

Study Participant \#

\section{CLCSS}

You will find below a series of statements, which describe how people feel about their lung cancer. Please use the scale below to describe to what extent each item is characteristic of you. Please circle a number ( 1 to 4$)$ that describes you best.

\begin{tabular}{|c|c|c|c|c|}
\hline & Strongly Agree & Agree & Disagree & $\begin{array}{r}\text { Strongly } \\
\text { Disagree } \\
\end{array}$ \\
\hline 1. I feel guilty because I have lung cancer. & 4 & 3 & 2 & 1 \\
\hline $\begin{array}{l}\text { 2. I work hard to keep my lung cancer a } \\
\text { secret. }\end{array}$ & 4 & 3 & 2 & 1 \\
\hline $\begin{array}{l}\text { 3. Having lung cancer makes me feel like I'm } \\
\text { a bad person. }\end{array}$ & 4 & 3 & 2 & 1 \\
\hline $\begin{array}{l}\text { 4. I'm very careful whom I tell I have lung } \\
\text { cancer. }\end{array}$ & 4 & 3 & 2 & 1 \\
\hline $\begin{array}{l}\text { 5. I feel I'm not as good as others because I } \\
\text { have lung cancer. }\end{array}$ & 4 & 3 & 2 & 1 \\
\hline 6. Having lung cancer makes me feel unclean. & 4 & 3 & 2 & 1 \\
\hline $\begin{array}{l}\text { 7. I feel set apart, isolated from the rest of the } \\
\text { world. }\end{array}$ & 4 & 3 & 2 & 1 \\
\hline $\begin{array}{l}\text { 8. My lung cancer diagnosis was delayed } \\
\text { because I put off going to the doctor. }\end{array}$ & 4 & 3 & 2 & 1 \\
\hline $\begin{array}{l}\text { 9. Someone told me lung cancer is what I } \\
\text { deserved for smoking. }\end{array}$ & 4 & 3 & 2 & 1 \\
\hline $\begin{array}{l}\text { 10. My lung cancer diagnosis was delayed } \\
\text { because my healthcare provider did not } \\
\text { take my "smoker's cough" seriously. }\end{array}$ & 4 & 3 & 2 & 1 \\
\hline $\begin{array}{l}\text { 11. Smokers could be refused treatment for } \\
\text { lung cancer. }\end{array}$ & 4 & 3 & 2 & 1 \\
\hline $\begin{array}{l}\text { 12. I have lost friends by telling them I have } \\
\text { lung cancer. }\end{array}$ & 4 & 3 & 2 & 1 \\
\hline $\begin{array}{l}\text { 13. I stopped socializing with some because of } \\
\text { their reactions. }\end{array}$ & 4 & 3 & 2 & 1 \\
\hline $\begin{array}{l}\text { 14. People have physically backed away from } \\
\text { me. }\end{array}$ & 4 & 3 & 2 & 1 \\
\hline
\end{tabular}


Study Participant \#

Strongly Agree

Agree

Disagree Strongly

Disagree

15. People I care about stopped calling after learning that I have lung cancer.

4

3

2

1

16. People seem afraid of me because I have lung cancer.

4

3

2

1

17. People avoid touching me if they know I have lung cancer.

4

3

2

1

18. People avoid you because lung cancer is associated with death.

4

3

2

1

19. Some people who know have grown more distant.

4

3

2

1

20. I was hurt how people reacted to learning I have lung cancer.

4

2

1

21. I worry about people discriminating against me.

$\begin{array}{llll}4 & 3 & 2 & 1\end{array}$

22. People with lung cancer are treated like outcasts.

4

3

2

23. Most people believe a person with lung cancer is dirty.

4

3

2

1

24. Most people are uncomfortable around someone with lung cancer.

4

3

2

1

25. I worry that people may judge me when they learn I have lung cancer.

4

3

2

1

26. People with lung cancer lose jobs when employers learn.

4

3

2

1

27. Lung cancer is viewed as a self-inflicted disease.

4

3

2

1

28. Others assume that a patient's lung cancer was caused by smoking, even if he or she never smoked.

4

3

2

1

29. Others assume that a patient's lung cancer was caused by smoking, even if he or she had stopped smoking years ago.

30. Some people act as though it is my fault that I have lung cancer.

4

4

3

2 
Study Participant \#

\begin{tabular}{lcccc} 
& Strongly Agree & Agree & Disagree & $\begin{array}{c}\text { Strongly } \\
\text { Disagree }\end{array}$ \\
\hline $\begin{array}{l}\text { 31. Healthcare providers don't take "smoker's } \\
\text { cough" seriously. }\end{array}$ & 4 & 3 & 2 & 1
\end{tabular}




\section{Appendix F}

\section{Modified Marlowe-Crowne Social Desirability Scale}

\begin{tabular}{|c|c|c|c|}
\hline \multirow{2}{*}{\multicolumn{4}{|c|}{ MMCSDS }} \\
\hline & & & \\
\hline \multicolumn{4}{|c|}{$\begin{array}{l}\text { Please read each statement and circle } T \text { for True or } F \text { for False. There are no right or wrong } \\
\text { answers. Do not spend too much time on any statement. }\end{array}$} \\
\hline 1 & I'm always willing to admit it when I make a mistake. & $\mathrm{T}$ & $\mathrm{F}$ \\
\hline 2 & I always try to practice what I preach. & $\mathrm{T}$ & $\mathrm{F}$ \\
\hline 3 & I never resent being asked to return a favor. & $\mathrm{T}$ & $\mathrm{F}$ \\
\hline 4 & $\begin{array}{l}\text { I have never been irked when people expressed ideas very different from my } \\
\text { own. }\end{array}$ & $\mathrm{T}$ & $\mathrm{F}$ \\
\hline 5 & I have never deliberately said something that hurt someone's feelings. & $\mathrm{T}$ & $F$ \\
\hline 6 & I like to gossip at times. & $\mathrm{T}$ & $\mathrm{F}$ \\
\hline 7 & There have been occasions when I took advantage of someone. & $\mathrm{T}$ & $\mathrm{F}$ \\
\hline 8 & I sometimes try to get even rather than forgive and forget. & $\mathrm{T}$ & $F$ \\
\hline 9 & At times I have really insisted on having things my own way. & $\mathrm{T}$ & $\mathrm{F}$ \\
\hline 10 & There have been occasions when I felt like smashing things. & $\mathrm{T}$ & $\mathrm{F}$ \\
\hline 11 & I never hesitate to go out of my way to help someone in trouble. & $\mathrm{T}$ & $\mathrm{F}$ \\
\hline 12 & I have never intensely disliked anyone. & $\mathrm{T}$ & $\mathrm{F}$ \\
\hline 13 & When I don't know something I don't at all mind admitting to it. & $\mathrm{T}$ & $\mathbf{F}$ \\
\hline 14 & I am always courteous, even to people who are disagreeable. & $\mathrm{T}$ & $\mathbf{F}$ \\
\hline 15 & I would never think of letting someone else be punished for my wrong doings. & $\mathrm{T}$ & $\mathrm{F}$ \\
\hline 16 & I sometimes feel resentful when I don't get my way. & $\mathrm{T}$ & $\mathrm{F}$ \\
\hline 17 & $\begin{array}{l}\text { There have been times when I felt like rebelling against people in authority } \\
\text { even though I knew they were right. }\end{array}$ & $\mathrm{T}$ & $\mathrm{F}$ \\
\hline 18 & I can remember "playing sick" to get out of something. & $\mathrm{T}$ & $\mathrm{F}$ \\
\hline 19 & There have been times when I was quite jealous of the good fortune of others. & $\mathrm{T}$ & $\mathrm{F}$ \\
\hline 20 & I am sometimes irritated by people who ask favors of me. & $\mathrm{T}$ & $F$ \\
\hline
\end{tabular}




\section{Appendix G}

\section{Timing of Help-Seeking Behavior and Demographic Questionnaire}

Timing of Help-Seeking Behavior \& Demographic Questionnaire

Study Participant \#

Date

[1] When did you first notice a change in your health? (month/year)

[2] What change in your health or symptom were you experiencing at that time?

[3] When did you first seek help for this change in health? (month/year)

[4] When did you receive help for this change in your health? (month/year)

[5] When were you diagnosed with lung cancer? (month/year)

[6] What stage of lung cancer were you diagnosed with initially?

[7] After your diagnosis, were you able to look back and notice more changes in your health that you did not realize before the diagnosis?

$\mathrm{Y} / \mathrm{N}$

If so, what were those symptoms?

[8] What is your age now (in years)?

[9] What was your age when you were diagnosed with lung cancer (in years)? 
[10] When you were diagnosed with lung cancer, what was your marital status?

$\begin{array}{lll}\text { Never Married } & \text { Separated } & \text { Widowed } \\ \text { Now Married } & \text { Divorced } & \end{array}$

[11] What is your current marital status?

Never Married Separated Widowed

Now Married (same spouse as in Question \#10)

Now Married (different spouse than in Question \#10)

Divorced

[12] Gender: Male / Female

[13] Which one or more of the following would you say your ethnicity is?
(a) White
(d) Native Hawaiian or Other Pacific Islander
(b) Black or African-American
(e) American Indian or Alaska Native
(c) Asian
(f) Other [specify]

[14] Employment Status:

Employed Full-Time Self-Employed Retired

Employed Part-Time Unemployed

[15] Occupation:

[16] When you were diagnosed with lung cancer, was your annual household income from all sources:

[1] Less than $\$ 25,000$ per year

[2] $\$ 25,000-\$ 50,000$ per year

[3] greater than $\$ 50,000$ per year

[17] Is your current annual household income from all sources:

[1] Less than $\$ 25,000$ per year

[2] $\$ 25,000-\$ 50,000$ per year

[3] greater than $\$ 50,000$ per year

[18] When you were diagnosed with lung cancer, did you have? 
[1] Medicare

[2] Medicare Supplement

[3] Medicaid

[4] Private Health Insurance

[5] Uninsured

[19] How would you describe your financial status when you were diagnosed?

[1] I have more than I need to live well.

[2] I have just about enough to get by.

[3] I sometimes struggle to make ends meet.

[20] How would you describe your current financial status?

[1] I have more than I need to live well.

[2] I have just about enough to get by.

[3] I sometimes struggle to make ends meet.

[21] What is the highest level of education you have completed?

[1] Less than High School

[2] High School Graduate

[3] Some College

[4] College Graduate or Higher

[22.1] Have you smoked at least 100 cigarettes in your entire life?

(NOTE: 5 packs $=100$ cigarettes)

[1] Yes

[2] No (If No, End questions here)

[3] Refused (Declined to Answer)

[22.2] At the time you were diagnosed with lung cancer, were you a smoker?

[1] Yes

If Yes, how many packs per day?

[2] No

[3] Refused (Declined to Answer)

[22.3] Do you now smoke cigarettes every day, some days, or not at all? 
[1] Every day

[2] Some days

[3] Not at all

[4] Refused (Declined to Answer)

[22.4] How long has it been since you last smoked cigarettes regularly?

[1] Within the past month (less than 1 month ago)

[2] Within the past 3 months (1 month but less than 3 months ago)

[3] Within the past 6 months ( 3 months but less than 6 months ago)

[4] Within the past year ( 6 months but less than 1 year ago)

[5] Within the past 5 years (1 year but less than 5 years ago)

[6] Within the past 10 years (5 years but less than 10 years ago)

[7] 10 years or more

[8] Never smoked regularly

[77] Don't know / Not sure

[99] Refused 


\title{
CURRICULUM VITAE
}

\author{
Lisa Carter-Harris, MSN, APRN, NP-C \\ 4031 Viewcrest Loop \\ Floyds Knobs, IN 47119 \\ (812) 923-8140 home \\ (502) 541-2405 cell \\ lisacarter.harris@louisville.edu
}

\section{Education}

2009 - present

Candidate, Doctor of Philosophy in Nursing

University of Louisville School of Nursing, Louisville, Kentucky

GPA 3.99

Anticipated graduation date: Summer 2013

2004

Master of Science in Nursing, Adult Nurse Practitioner

University of Louisville School of Nursing, Louisville, Kentucky

1999

Bachelor of Science in Nursing

Spalding University School of Nursing, Louisville, Kentucky

1994

Associate of Arts, General Studies

University of Maryland - European Division, Berlin, Germany

\section{Certifications \& Licensure}

Adult Nurse Practitioner, Board Certified, American Academy of Nurse Practitioners (06.2004 present)

Advanced Practice Registered Nurse (Nurse Practitioner), Kentucky License 30004352

Registered Nurse, Kentucky License 1091288

Advanced Registered Nurse Practitioner, Indiana License 71003273A

Registered Nurse, Indiana License 28158514A

American Heart Association BLS for Healthcare Providers, 1999 - present

Professional Employment

2010 - present Nurse Practitioner

Advanced Healthcare Associates, Louisville, KY

2010 - present Lecturer

University of Louisville School of Nursing, Louisville, KY

2010-2012 Clinical Assistant Professor

Indiana University Southeast, New Albany, IN

2010-2011 Nurse Practitioner/Practice Owner

MyCare Family Clinic, Shively, KY

$2007-2011$

Nurse Practitioner/Owner

LCH Healthcare Options, PLLC, Louisville, KY 
Summer 2011 \&

$2004-2005$

Sum. 2012 \& 2011 Adjunct Faculty (Graduate Program)

Adjunct Faculty (Undergraduate Program)

Bellarmine University School of Nursing, Louisville, KY

Spalding University School of Nursing, Louisville, KY

$2004-2007$

Nurse Practitioner

Rheumatology Associates, Louisville, KY

$2004-2005$

$2003-2004$

$2001-2003$

$2000-2001$

$1999-2000$
Adjunct Faculty (Undergraduate Program)

Spalding University School of Nursing, Louisville, KY

Staff Nurse, Labor \& Delivery

Norton Suburban Hospital, Louisville, KY

Staff Nurse, Labor \& Delivery

Baptist Hospital East, Louisville, KY

Charge Nurse, Emergency Department

Caritas Medical Center, Louisville, KY

Staff Nurse, Emergency Department \& ICU/CCU

University of Louisville Hospital, KY

\section{Professional Awards, Honors and Scholarships}

2012 - 2013 Sponsored Research Tuition Dissertation Grant University of Louisville School of Interdisciplinary Graduate Studies

2012 Graduate Nursing Student Poster Award Winner, Research Louisville, Help-Seeking Behaviors in Lung Cancer

2012 KYANNA Black Nurses Association of Louisville Excellence in Nursing Award for Research

2011 Kentucky Coalition of Nurse Practitioners and Nurse Midwives Doctoral Scholarship Award

$2011 \quad$ University of Louisville Faculty Favorite Award

2010 - 2012 American Association of Colleges of Nursing/Johnson \& Johnson Minority Nursing Faculty Scholar

2010 Golden Key International Honor Society inductee

$2009-2010$ Professional Nurse Traineeship, University of Louisville School of Nursing

2004 Dean's Award for Nursing Excellence, University of Louisville 
1999 Who's Who in American Universities \& Colleges

1999 Baccalaureate Speaker, Spalding University Graduation

1999 Mother Rose Meagher University-wide Award, Spalding University

1999 Payne Award, Spalding University School of Nursing

\section{Professional Memberships and Activities}

AcademyHealth, member since 2010

American Academy of Nurse Practitioners, member since 2003

American Nurses Association, member since 1999

KYANNA Black Nurses Association of Louisville/NBNA, member since 2011

Kentucky Coalition of Nurse Practitioners and Nurse Midwives, member since 2003

Sigma Theta Tau International Nursing Honor Society, Spalding University, inducted and member since 1997

- Nominating Chair, lota Gamma Chapter, 2010-2011 term

- President, lota Gamma Chapter, 2004-2006 term

- lota Zeta Chapter, member since 2012

Midwest Nursing Research Society, member since 2012

Southern Nursing Research Society, member since 2012

\section{Presentations}

- Help-Seeking Behaviors in Lung Cancer, Student Poster Presentation, Research Louisville, September 2012

- Help-Seeking Behaviors in Lung Cancer, Student Poster Presentation, Southern Nursing Research Society Annual Conference, New Orleans, LA, February 2012

- Health-Seeking Behaviors in Lung Cancer, Student Poster \& Podium Presentation, Annual Research Colloquium, University of Louisville School of Nursing, February 2012

- Advanced Practice Nursing, Guest Speaker, Indiana University Southeast Senior Nursing Students (January 2012)

- Transition to Advanced Practice, Guest Speaker, University of Louisville NP Students (February 2011)

- Advanced Practice Panel Speaker, Spalding University School of Nursing Graduate Program (May 2010)

- Advanced Nursing Practice, Spalding University School of Nursing Senior Class (October 2008 and January 2009)

- Rheumatology 101 for Nurse Practitioners, Bellarmine University Graduate Students (June and November 2012)

- Rheumatology 101 for Nurse Practitioners, University of Louisville Graduate Students (January 2007, November 2009, December 2010, and November 2012) 
- Rheumatology 101 for Nurse Practitioners, Spalding University Graduate Students (October 2006 and October 2007)

- Osteoporosis: Getting Down to the Nitty Gritty of Bone Health - Kentucky Governor's Conference on Women's Health, Invited Speaker, Kentucky International Convention Center, July 2007

\section{Service}

Indiana University Southeast

2012

2011

2010

Improvement of Learning Committee

Common Experience Committee

Common Experience Committee

2010

Faculty Learning Community on Diversity

Community Service

2012 - present Indiana Health Policy Coordinator - Lung Cancer

2011

Alliance/Southern Indiana-Kentucky Chapter

Passport Health Plan Pharmacy \& Therapeutics Committee

Teaching Experience

Spring 2013

University of Louisville

- NURS 360-50 - Physical Health Assessment for RN to BSN (Online Didactic Course)

- NURS 384-50 - Global Health Nursing (Online Didactic Course)

Fall 2012

University of Louisville

- NURS 360-50 - Physical Health Assessment for RN to BSN (Online Didactic Course)

- NURS 382-50 - Nursing Research for Evidence-Based Practice (Online Didactic Course)

- Clinical Faculty for 4 Graduate Nurse Practitioner Students (Adult Primary Care)

Summer 2012

Indiana University - RN to BSN Online Program

- K301 - Complementary and Alternative Medicine

Spalding University

- NURS 657 - Adult Primary Care for Nurse Practitioners

\section{Spring 2012}

Indiana University Southeast

- B248 - Science and Technology Didactic Course

- B249 - Science and Technology Practicum 
- H362 - Alterations in Health II Practicum

- H363 - Developing Family Practicum

University of Louisville

- NURS 360-50 - Physical Health Assessment for RN to BSN (Online Didactic Course)

- Clinical Faculty for 4 Graduate Nurse Practitioner Students (Adult Primary Care)

\section{Fall 2011}

Indiana University Southeast

- B244 - Comprehensive Health Assessment Didactic Course

- B245 - Health Assessment Practicum (two sections)

- H354 - Alterations in Health I Practicum

University of Louisville

- Clinical Faculty for 6 Graduate Nurse Practitioner Students (Adult Primary Care)

\section{Summer 2011}

Bellarmine University

- NURS 209 - Foundations of Nursing Practice (didactic and practicum)

Spalding University

- NURS 657 - Adult Primary Care

\section{Spring 2011}

Indiana University Southeast

- H362 - Alterations in Health II Practicum

- H363 - Developing Family Practicum

University of Louisville

- NURS 657-50 - Health Promotion (Graduate Program)

\section{Fall 2010}

Indiana University Southeast

- B245 - Health Assessment Practicum (two sections)

- $\mathrm{S} 473$ - Health of the Community: Practicum

University of Louisville

- NURS 657-50 - Health Promotion (Graduate Program)

- NURS 382-50 - Nursing Research for Evidence-Based Practice (RN to BSN Program)

- Clinical Faculty for 6 Graduate Nurse Practitioner Students (Adult Primary Care) 